Rev. Mat. Iberoamericana 26 (2010), no. 2, 367-448

\title{
Convergence of metric graphs and energy forms
}

\begin{abstract}
In this paper, we begin with clarifying spaces obtained as limits of sequences of finite networks from an analytic point of view, and we discuss convergence of finite networks with respect to the topology of both the Gromov-Hausdorff distance and variational convergence called $\Gamma$-convergence. Relevantly to convergence of finite networks to infinite ones, we investigate the space of harmonic functions of finite Dirichlet sums on infinite networks and their Kuramochi compactifications.
\end{abstract}

\section{Introduction}

A finite (resistive) network consists of the set of vertices, the set of edges, and the resistance that assigns a positive number to each edge. Regarding the resistance of each edge as its length, we can introduce a distance, called the geodesic distance or the path metric, on the set of vertices. On the other hand, using a canonical energy form on the space of functions on the vertices, we have a notion of the effective resistance between a pair of vertices. It is well known that the effective resistance provides the set of vertices with another metric called the resistance metric in Kigami [32], where convergence of finite networks with the resistance metrics in a specific manner is discussed and applied to the problem of constructing energy forms and Laplacians on certain fractal sets called post critically finite self-similar sets. On the other hand, infinite networks may be thought to be limits of sequences of finite networks with the geodesic distances or the resistance metrics. Moreover it is known that any compact geodesic space can be approximated by a

2000 Mathematics Subject Classification: Primary: 31C20. Secondary: 53C23, 60J10. Keywords: Network, resistance form, resistance metric, Gromov-Hausdorff convergence, $\Gamma$-convergence, harmonic function of finite Dirichlet sum, Kuramochi compactification. 
sequence of finite networks with the geodesic distances with respect to the Gromov-Hausdorff topology.

In this paper, we begin with adapting the general theory on Hilbert spaces possessing reproducing kernels by Aronszajn [1] for our purpose, and then, introducing resistance forms, we clarify spaces obtained as limits of finite networks which are investigated in Kigami [32]. A separable space endowed with a resistance form can be considered as the limit of finite subsets coupled with the traces of the resistance form on them. Limits of finite networks can be also taken in a similar manner to the Gromov-Hausdorff convergence of metric spaces.

A pointed metric space $(X, p)$ is a pair of a metric space $X$ and a point $p \in X$. We say that a sequence $\left\{\left(X_{n}, p_{n}\right)\right\}$ of pointed metric spaces converges to a pointed metric space $(X, p)$ in the Gromov-Hausdorff sense if the following holds. For every $\rho>0$ and $\varepsilon>0$, there exists a positive integer $n_{0}$ such that for any $n>n_{0}$, there is a map $f$ from the ball $B_{\rho}\left(p_{n}\right)$ around $p_{n}$ with radius $\rho$ in $X_{n}$ to $X$ satisfying the following properties: (i) $f\left(p_{n}\right)=p$; (ii) $\sup \left\{\left|d_{X}\left(f\left(x_{1}\right), f\left(x_{2}\right)\right)-d_{X_{n}}\left(x_{1}, x_{2}\right)\right| \mid x_{1}, x_{2} \in B_{\rho}\left(p_{n}\right)\right\}<\varepsilon$;(iii) the $\varepsilon$ neighborhood of the set $f\left(B_{\rho}\left(p_{n}\right)\right)$ includes the ball $B_{\rho-\varepsilon}(p)$ centered at $p$ of radius $\rho-\varepsilon$.

Let $(X, R)$ and $\left\{\left(\Gamma_{n}, R_{n}\right)\right\}$ be a separable metric space and a sequence of connected, finite networks with the resistance metrics $R_{n}$. Let $p$ and $p_{n}$ be points of $X$ and $V_{n}$ respectively. Then it will be shown (cf. Theorem 5.1) that the distance $R$ of $X$ is the resistance metric associated to a resistance form on $X$ if the pointed metric space $\left(\Gamma_{n}, R_{n}, p_{n}\right)$ converges to $(X, R, p)$ as $n \rightarrow \infty$.

Now we notice that the effective resistance between two vertices in a connected network is less than or equal to the geodesic distance between them. A locally finite graph is always assumed to be a network with unit resistance. Taking this into account, we will discuss convergence of finite graphs with geodesic distances and prove the following result (cf. Theorem 5.3): Let $\left\{\left(G_{n}, d_{n}\right)\right\}$ be a sequence of connected, finite graphs endowed with the geodesic distance $d_{n}$, and let $\left(G, d_{G}\right)$ be a connected, locally finite, infinite graph with the geodesic distance $d_{G}$. Suppose that the pointed metric space $\left(G_{n}, d_{n}, p_{n}\right)$ converges, as $n \rightarrow \infty$, to the pointed metric space $\left(G, d_{G}, p\right)$ in the Gromov-Hausdorff sense. Then there exist a subsequence $\left\{G_{m}\right\}$ and a resistance form $\mathcal{E}$ on $G$ such that the pointed metric space $\left(G_{m}, R_{m}, p_{m}\right)$ with the resistance metric $R_{m}$ converges, as $m \rightarrow \infty$, to the pointed metric space $\left(G, R_{\mathcal{E}}, p\right)$ with the resistance metric $R_{\mathcal{E}}$ relative to the resistance form $\mathcal{E}$ in a similar manner to the above; moreover the from $(\mathcal{E}, D[\mathcal{E}])$ satisfies that $D_{0}\left[\mathcal{E}_{G}\right] \subset D[\mathcal{E}] \subset D\left[\mathcal{E}_{G}\right], \mathcal{E}(u) \geq \mathcal{E}_{G}(u)$ for all $u \in D[\mathcal{E}]$, and $\mathcal{E}(u, v)=\mathcal{E}_{G}(u, v)$ for all $u \in D[\mathcal{E}]$ and $v \in D_{0}\left[\mathcal{E}_{G}\right]$. 
Here on an infinite network $\Gamma$, we have two canonical resistance forms of Dirichlet sums (of order 2), the minimal and the maximal ones denoted by $\left(\mathcal{E}_{\Gamma}^{0}, D_{0}\left[\mathcal{E}_{\Gamma}\right]\right)$ and $\left(\mathcal{E}_{\Gamma}, D\left[\mathcal{E}_{\Gamma}\right]\right)$ respectively. If the network is parabolic or it admits no nonconstant harmonic functions of finite Dirichlet sums, we have the uniqueness of limit forms. However, in the case that $\Gamma$ admits nonconstant harmonic functions of finite Dirichlet sums, limit forms may be different from the minimal and the maximal ones.

For instance, given a family of expanders, namely, a family of connected finite graphs whose degrees are uniformly bounded from above and whose spectral gaps are uniformly bounded away from zero, we can take a subsequence converging to an infinite graph in such a way that the limit form is the minimal one; moreover starting with a certain family of expanders and modifying it in certain manners, we will be able to get a subsequence which converges to an infinite graph in such a way that the limit form is distinct from the minimal and the maximal ones (cf. 7.1). In fact, the topology of the Gromov-Hausdorff convergence on resistance metrics is finer than that on geodesic distances.

Relevantly to convergence of finite networks to an infinite one, we investigate in section 7 the Kuramochi compactification of an infinite network and the trace of the resistance form on the Kuramochi boundary. For example, it is proved that the Kuramochi compactification of a connected, infinite network inherits the resistance form if the diameter of the resistance metric is finite (cf. Theorems 3.11 and 7.11). To study the compactification, we employ the method of embedding networks into the Hilbert space of square summable sequences by using the eigenvalues and eigenfunctions with respect to certain self-adjoint operators. This method proves useful for studying networks and their limit spaces.

Bérard, Besson and Gallot [5, 6] proposed the method of embedding compact Riemannian manifolds into the Hilbert space above by using their eigenvalues and eigenfunctions, and introduced a distance on the set of their isometry classes, proving the precompactness of a family of compact $n$ dimensional Riemannian manifolds whose Ricci curvature are uniformly bounded from below and whose diameters are uniformly bounded from above. In [29], [30], [26] and [27], the method was developed to study spectral convergence of compact Riemannian manifolds or more generally certain Dirichlet spaces, and analyze their limit spaces.

Convergence of finite networks can be also discussed from a variational view point and in fact we will apply the idea of De Giorgi's $\Gamma$-convergence to our problem (cf. Theorem 5.5).

Besides the relation to problems on convergence of networks, we are interested in the space of harmonic functions of finite Dirichlet sums on an 
infinite network. When we restrict ourselves to a class of connected, infinite graphs of bounded degrees, it is proved in [24] that this space is invariant under quasi-isometries (cf. Remark 1.2) in the sense that quasi-isometries between two connected infinite graphs of bounded degrees canonically induce bounded linear isomorphisms between the spaces of harmonic functions of finite Dirichlet sums; this is true for quasi-isometries between a connected infinite graph of bounded degrees and a connected, complete, noncompact Riemannian manifold such that the Ricci curvature is bounded from below and the volume of every ball of radius one is bounded from below by a positive constant. In Sections 4 and 7, we make some observations on spaces of functions of finite Dirichlet sums in relation to resistance metrics on infinite networks (cf. Proposition 4.1, Theorem 4.2, Theorem 7.11).

Some of the main results of this paper were reported in [28].

\section{Hilbert spaces of reproducing kernels}

We first adapt the general theory of Hilbert spaces possessing reproducing kernels in [1] for our later purpose.

1.1 Let $X$ be a set and $(\mathcal{E}, D[\mathcal{E}])$ a nonnegative quadratic form $\mathcal{E}$ defined on a linear subspace $D[\mathcal{E}]$ of the space $\ell(X)$ of all real valued functions on $X$. Let

$$
R_{\mathcal{E}}(x, y)=\sup \left\{\frac{|u(x)-u(y)|^{2}}{\mathcal{E}(u, u)} \mid u \in D[\mathcal{E}], \mathcal{E}(u, u)>0\right\}, \quad x, y \in X .
$$

Our basic condition on the form is stated in the following:

$[\mathrm{H}-1] \quad 0<R_{\mathcal{E}}(x, y)<+\infty \quad$ for all $x, y \in X$ with $x \neq y$.

The form $\mathcal{E}$ is also regarded as a functional on $\ell(X)$ by letting $\mathcal{E}(u)=$ $\mathcal{E}(u, u)$ if $u \in D[\mathcal{E}]$ and $\mathcal{E}(u)=+\infty$ otherwise. From this point of view, we consider the following:

[H-2] if a sequence of functions $u_{n}$ on $X$ pointwise converges to a function $u$ as $n \rightarrow \infty$, we have

$$
\mathcal{E}(u) \leq \liminf _{n \rightarrow \infty} \mathcal{E}\left(u_{n}\right) \leq+\infty .
$$

Lemma 1.1 Under condition [H-1], [H-2] implies the following:

[H-3] for a point $o \in X$, a quadratic form defined by $\mathcal{E}(u, v)+u(o) v(o)$ provides a complete inner product on $D[\mathcal{E}]$, that is, $\left(D[\mathcal{E}], \mathcal{E}+\delta_{o}^{2}\right)$ is a Hilbert space; this holds for any $o \in X$ because of [H-1], where $\delta_{o}$ stands for the linear functional on $\ell(X)$ defined by $\delta_{o}(u)=u(o)$ for $u \in \ell(X)$. 
Proof. Let $\left\{u_{n}\right\}$ be a Cauchy sequence in $\left(D[\mathcal{E}], \mathcal{E}+\delta_{o}^{2}\right)$. Since

$$
\left|u_{m}(x)-u_{n}(x)\right|^{2} \leq 2 R_{\mathcal{E}}(x, o) \mathcal{E}\left(u_{m}-u_{n}\right)+2 \delta_{o}\left(u_{m}-u_{n}\right)^{2}, \quad x \in X,
$$

$u_{n}$ converges pointwise to a function $u$ on $X$. Hence by [H-2], we have

$$
\mathcal{E}(u) \leq \liminf _{n \rightarrow \infty} \mathcal{E}\left(u_{n}\right)<+\infty ;
$$

in particular, $u \in D[\mathcal{E}]$. Since $u_{n}-u_{m}$ pointwise converges to $u_{n}-u$ as $m \rightarrow \infty$, we thus obtain

$$
\limsup _{n \rightarrow \infty} \mathcal{E}\left(u_{n}-u\right) \leq \limsup _{n \rightarrow \infty} \liminf _{m \rightarrow \infty} \mathcal{E}\left(u_{n}-u_{m}\right)=0 .
$$

This shows that the inner product $\mathcal{E}+\delta_{o}^{2}$ is complete.

The proof above yields the following

Lemma 1.2 Suppose, in addition to [H-1], that $\sup _{x, y \in X} R_{\mathcal{E}}(x, y)$ is bounded. Then $[\mathrm{H}-3]$ is implied by the following:

[H-2'] for a sequence of bounded functions $u_{n}$ on $X$ that uniformly converges to a bounded function $u$, that is, $\lim _{n \rightarrow \infty} \sup _{x \in X}\left|u_{n}(x)-u(x)\right|=0$, we have

$$
\mathcal{E}(u) \leq \liminf _{n \rightarrow \infty} \mathcal{E}\left(u_{n}\right) \leq+\infty .
$$

Now we consider the case where [H-1] and [H-3] are verified. Let $K$ be a nonempty subset of $X$ and denote by $D[\mathcal{E} ; K]$ the space of functions $u \in D[\mathcal{E}]$ such that $u(z)=0$ for all $z \in K$. Then the restriction of $\mathcal{E}$ to $D[\mathcal{E} ; K]$ provides a complete inner product, and for any $x \in X$, we have

$$
|u(x)|^{2} \leq R_{\mathcal{E}}(z, x) \mathcal{E}(u), \quad u \in D[\mathcal{E} ; K], z \in K,
$$

which implies that for any $x \in X$, there exists uniquely a function $g_{K ; x} \in$ $D[\mathcal{E} ; K]$, the reproducing kernel of $D[\mathcal{E} ; K]$, satisfying

$$
u(x)=\mathcal{E}\left(g_{K ; x}, u\right), \quad u \in D[\mathcal{E} ; K] .
$$

We write $g_{K}(x, y)$ instead of $g_{K ; x}(y)$ for $x, y \in X$. Since $\mathcal{E}$ is symmetric, we see that $g_{K}(x, y)=g_{K}(y, x)$. In the case where $K$ consists of a single point $z, g_{z}(x, y)$ stands for $g_{\{z\}}(x, y)$. We note that $g_{K}(x, x)=$ $\mathcal{E}\left(g_{K}(x, *), g_{K}(x, *)\right) \leq g_{z}(x, x)=\mathcal{E}\left(g_{z}(x, *), g_{z}(x, *)\right)$ for all $z \in K$.

Let $\left\{u_{n}\right\}$ be a sequence in $D[\mathcal{E} ;\{p\}]$ for a point $p \in X$, and assume that $\mathcal{E}\left(u_{n}\right)$ is bounded. Then a subsequence $\left\{u_{m}\right\}$ weakly converges to a function $u$ in $D[\mathcal{E} ;\{p\}]$ as $m \rightarrow \infty$, that is, $\mathcal{E}\left(u_{m}, v\right)$ tends to $\mathcal{E}(u, v)$ for all $v \in D[\mathcal{E} ;\{p\}]$. By taking $v=g_{p}(x, *), x \in X$, we see that $u_{m}(x)$ converges to $u(x)$ as $m \rightarrow \infty$, and thus $u_{m}$ converges to $u$ pointwise on $X$. In this way, we have the following 
Lemma 1.3 Suppose that $[\mathrm{H}-1]$ and $[\mathrm{H}-3]$ hold. Let $\left\{u_{n}\right\}$ be a sequence in $D[\mathcal{E} ;\{p\}]$ for a point $p \in X$ such that $\mathcal{E}\left(u_{n}\right)$ is bounded as $n \rightarrow \infty$. Then $u_{n}$ weakly converges to a function $u$ in $D[\mathcal{E} ;\{p\}]$ as $n \rightarrow \infty$ if and only if $u_{n}$ converges pointwise to a function $u \in \ell(X)$ as $n \rightarrow \infty$. Moreover in these cases, one has

$$
\mathcal{E}(u) \leq \liminf _{n \rightarrow \infty} \mathcal{E}\left(u_{n}\right)<+\infty .
$$

Lemma 1.4 Suppose, in addition to [H-1] and [H-3], that

[H-4] $1 \in D[\mathcal{E}]$ and $\mathcal{E}(1,1)=0$.

Then the following assertions hold:

(i) [H-2] is verified; moreover for a sequence of $u_{n} \in D[\mathcal{E}]$ such that $\mathcal{E}\left(u_{n}\right)$ is bounded as $n \rightarrow \infty, u_{n}$ weakly converges to a function $u$ in $D[\mathcal{E}]$ and $u_{n}(x)$ tends to $u(x)$ as $n \rightarrow \infty$ for some $x \in X$ if and only if $u_{n}$ converges pointwise to a function $u \in \ell(X)$ as $n \rightarrow \infty$.

(ii) For all $x, y \in X, R_{\mathcal{E}}(x, y)=g_{x}(y, y)=g_{y}(x, x)$.

Proof. For any $u \in D[\mathcal{E}]$ and each $x \in X, u-u(x)$ belongs to $D[\mathcal{E} ;\{x\}]$ and $\mathcal{E}(u-u(x))=\mathcal{E}(u)$. This, together with Lemma 1.3, proves the first assertion. Moreover for any $y \in X$, we have $|u(y)-u(x)|^{2}=\mathcal{E}\left(g_{x}(y, *), u-\right.$ $u(x))^{2}=\mathcal{E}\left(g_{x}(y, *), u\right)^{2} \leq \mathcal{E}\left(g_{x}(y, *)\right) \mathcal{E}(u)=g_{x}(y, y) \mathcal{E}(u) ;$ thus $R_{\mathcal{E}}(x, y) \leq$ $g_{x}(y, y)$. On the other hand, by letting $u=g_{x}(y, *)$, we get $g_{x}(y, y)^{2} \leq$ $R_{\mathcal{E}}(x, y) \mathcal{E}\left(g_{x}(y, *)\right)=R_{\mathcal{E}}(x, y) g_{x}(y, y)$, and hence $g_{x}(y, y) \leq R_{\mathcal{E}}(x, y)$. Thus the second assertion is verified.

Now instead of condition [H-1], we assume that a form $\mathcal{E}$ under consideration satisfies

$[\mathrm{H}-5] \quad M_{\mathcal{E}}(x):=\sup \left\{\frac{|u(x)|^{2}}{\mathcal{E}(u)} \mid u \in D[\mathcal{E}], \mathcal{E}(u)>0\right\}<+\infty \quad$ for all $x \in X$.

As in Lemma 1.1, it is also evident that [H-2] verifies the completeness of the form $\mathcal{E}$ on $D[\mathcal{E}]$. On the other hand, if the latter is the case, then we have the reproducing kernel or the Green function $g_{\mathcal{E}}: X \times X \rightarrow \mathbb{R}$ of $\mathcal{E}$ satisfying

$$
\mathcal{E}\left(g_{\mathcal{E}}(x, *), u\right)=u(x), \quad u \in D[\mathcal{E}], x \in X .
$$

Using the Green function, we can verify [H-2]. Also it follows that $M_{\mathcal{E}}(x)=$ $g_{\mathcal{E}}(x, x)$ for all $x \in X$. Thus we have the following

Lemma 1.5 Under [H-5], [H-2] holds true if and only if $\mathcal{E}$ provides a complete inner product on $D[\mathcal{E}]$. Moreover in this case, for a sequence of $u_{n} \in$ $D[\mathcal{E}]$ with $\sup \mathcal{E}\left(u_{n}\right)<+\infty, u_{n}$ weakly converges to a function $u$ in $D[\mathcal{E}]$ and $u_{n}(x)$ tends to $u(x)$ as $n \rightarrow \infty$ for some $x \in X$ if and only if $u_{n}$ converges pointwise to a function $u \in \ell(X)$ as $n \rightarrow \infty$, and also one has $M_{\mathcal{E}}(x)=g_{\mathcal{E}}(x, x)$ for all $x \in X$. 
In what follows, we assume that $(X, \mathcal{E})$ satisfies conditions $[\mathrm{H}-1],[\mathrm{H}-2]$ and [H-4], or conditions [H-5] and [H-2], unless otherwise stated.

Definition 1.1 Given a nonempty subset $K$ of $X$ and a function $u$ on $K$, we set

$$
\mathcal{A}_{u}:=\left\{v \in D[\mathcal{E}] \mid v_{\mid K}=u\right\}, \quad D\left[\mathcal{E}_{K}^{*}\right]:=\left\{u \in \ell(K) \mid \mathcal{A}_{u} \neq \emptyset\right\},
$$

and

$$
\mathcal{E}_{K}^{*}(u):=\inf \left\{\mathcal{E}(v) \mid v \in \mathcal{A}_{u}\right\} .
$$

When $\mathcal{A}_{u}$ is empty, we understand $\mathcal{E}_{K}^{*}(u)=+\infty$. The induced form $\mathcal{E}_{K}^{*}$ on $\ell(K)$ is called the trace of $\mathcal{E}$ on $K$.

Lemma 1.6 Given $u \in D\left[\mathcal{E}_{K}^{*}\right]$, there exists a unique minimizer, denoted by $H_{K ; u}$, of $\mathcal{E}$ in $\mathcal{A}_{u}$. The minimizer is characterized as a function such that $H_{K ; u}=u$ on $K$ and $\mathcal{E}\left(H_{K ; u}, v\right)=0$ for all $v \in D[\mathcal{E}]$ that vanish on $K$.

Proof. Let $\left\{v_{n}\right\}$ be a minimizing sequence in $\mathcal{A}_{u}$. Passing to a subsequence, we may assume that $v_{n}$ weakly converges to a function $v \in D[\mathcal{E}]$ as $n \rightarrow \infty$ and hence, by Lemma 1.3, $v_{n}$ converges to $v$ pointwise in $X$. Thus it follows that $\mathcal{E}_{K}^{*}(u) \leq \mathcal{E}(v) \leq \liminf _{n \rightarrow \infty} \mathcal{E}\left(v_{n}\right)=\mathcal{E}_{K}^{*}(u)$ and $v$ belongs to $\mathcal{A}_{u}$. This shows that $v$ is a minimizer in $\mathcal{A}_{u}$. We observe that a minimizer $v$ in $\mathcal{A}_{u}$ satisfies $\mathcal{E}(v, w)=0$ for all $w \in D[\mathcal{E}]$ vanishing on $K$, since $\mathcal{E}(v+t w, v+t w) \geq$ $\mathcal{E}(v, v)$ for all real numbers $t$. Moreover such a function $v$ is unique. In fact, for minimizers $v_{1}$ and $v_{2}$ in $\mathcal{A}_{u}, \mathcal{E}\left(v_{1}-v_{2}, v_{1}-v_{2}\right)=\mathcal{E}\left(v_{1}, v_{1}-v_{2}\right)-\mathcal{E}\left(v_{2}, v_{1}-\right.$ $\left.v_{2}\right)=0$. This implies that $v_{1}-v_{2}$ is constant in $X$ and hence $v_{1}-v_{2}=0$. Finally for a function $v \in \mathcal{A}_{u}$, we have

$$
\mathcal{E}(v)=\mathcal{E}\left(v-H_{K ; u}\right)+\mathcal{E}\left(H_{K ; u}\right) \geq \mathcal{E}\left(H_{K ; u}\right) .
$$

This completes the proof of Lemma 1.6.

Lemma 1.7 The trace $\mathcal{E}_{K}^{*}$ on $K$ inherits the properties [H-1], [H-2] and $[\mathrm{H}-4]$, or the properties $[\mathrm{H}-5]$ and $[\mathrm{H}-2]$ from $\mathcal{E}$.

Proof. It suffices to prove that $\mathcal{E}_{K}^{*}+\delta_{o}^{2}$ provides a complete inner product on $D\left[\mathcal{E}_{K}^{*}\right]$ for any fixed point $o$ of $K$ if $[\mathrm{H}-1]$ is verified, and so does $\mathcal{E}_{K}^{*}$ if $[\mathrm{H}-5]$ is satisfied. In fact, if a sequence $\left\{u_{n}\right\}$ is fundamental in $D\left[\mathcal{E}_{K}^{*}\right]$, then $\left\{H_{K ; u_{n}}\right\}$ is so in $D[\mathcal{E}]$, and thus it converges to a function $H \in D[\mathcal{E}]$. Let $u=H_{\mid K}$. Then in view of Lemma 1.6, we have $H=H_{K ; u}$, since $\mathcal{E}(H, w)=\lim _{n \rightarrow \infty} \mathcal{E}\left(H_{K ; u_{n}}, w\right)=0$ for all $w \in D[\mathcal{E}]$ that vanish on $K$.

Lemma 1.8 Let $K$ and $L$ be two subsets of $X$ such that $K \subset L$. Then one has

$$
\mathcal{E}_{K}^{*}\left(u_{\mid K}\right) \leq \mathcal{E}_{L}^{*}(u)
$$

for $u \in D\left[\mathcal{E}_{L}^{*}\right]$, and the equality holds if and only if $H_{K ; u_{\mid K}}=u$ on $L$, that is, $H_{K ; u_{\mid K}}=H_{L ; u}$ on $X$. 
Proof. For $u \in D\left[\mathcal{E}_{L}^{*}\right]$, it is easy to see that $\mathcal{E}_{L}^{*}(u, v)=0$ for any $v \in D\left[\mathcal{E}_{L}^{*}\right]$ vanishing on $K$ if and only if $\mathcal{E}\left(H_{L ; u}, w\right)=0$ for every $w \in D[\mathcal{E}]$ vanishing on $K$. This, together with the definition of the traces, shows the lemma.

Now as in the case of the functional $\mathcal{E}: \ell(X) \rightarrow[0,+\infty]$, we set

$$
R_{\mathcal{E}_{K}^{*}}(x, y)=\sup \left\{\frac{|u(x)-u(y)|^{2}}{\mathcal{E}_{K}^{*}(u)} \mid u \in D\left[\mathcal{E}_{K}^{*}\right], \mathcal{E}_{K}^{*}(u) \neq 0\right\}, \quad x, y \in K
$$

if $[\mathrm{H}-1]$ is satisfied, and also

$$
M_{\mathcal{E}_{K}^{*}}(x)=\sup \left\{\frac{|u(x)|^{2}}{\mathcal{E}_{K}^{*}(u)} \mid u \in D\left[\mathcal{E}_{K}^{*}\right], \mathcal{E}_{K}^{*}(u)>0\right\}, \quad x \in K
$$

if [H-5] is verified. Since it follows from Lemma 1.8 that the Green functions $g_{z}(z \in X)$ of $\mathcal{E}$ coincide with those of $\mathcal{E}_{K}^{*}$ on $K \times K$ if $z \in K$, we have the following

Lemma 1.9 (i) For all $x, y \in K$, one has $R_{\mathcal{E}_{K}^{*}}(x, y)=R_{\mathcal{E}}(x, y)$ if $\mathcal{E}$ satisfies [H-1], [H-2] and [H-4].

(ii) For all $x \in K, M_{\mathcal{E}_{K}^{*}}(x)=M_{\mathcal{E}}(x)$ if [H-5] and [H-2] are verified.

In what follows, in addition to [H-1], [H-2] and [H-4], or [H-5] and [H-2], we always assume the following condition:

[H-6] there is a distance $d$ on $X$ such that the metric space $(X, d)$ is separable and every function of $D[\mathcal{E}]$ is continuous on the space, that is, $D[\mathcal{E}] \subset$ $C(X, d)$.

Lemma 1.10 Let $\left\{X_{n}\right\}$ be an increasing sequence of finite subsets $X_{n}$ of $X$ such that the union $\cup_{n} X_{n}$ is dense in $X$. Then one has

$$
\begin{aligned}
& D[\mathcal{E}]=\left\{u \in C(X, d) \mid \lim _{n \rightarrow \infty} \mathcal{E}_{X_{n}}^{*}\left(u_{\mid X_{n}}\right)<+\infty\right\} ; \\
& \mathcal{E}(u)=\lim _{n \rightarrow \infty} \mathcal{E}_{X_{n}}^{*}\left(u_{\mid X_{n}}\right), u \in D[\mathcal{E}] .
\end{aligned}
$$

Proof. Let $h_{n}=H_{X_{n} ; u_{\mid X_{n}}}$ for simplicity. Then $\left\{\mathcal{E}\left(h_{n}\right)\right\}$ is an increasing sequence and $\lim _{n \rightarrow \infty} \mathcal{E}\left(h_{n}\right) \leq \mathcal{E}(u) \leq+\infty$. Suppose that $\lim _{n \rightarrow \infty} \mathcal{E}\left(h_{n}\right)<+\infty$. Then in view of Lemma 1.3, we can find a subsequence $\left\{h_{m}\right\}$ which pointwise converges to a function $h$ on $X$ as $m \rightarrow \infty$. Obviously $h=u$ on $\cup_{n} X_{n}$ and hence everywhere on $X$, since $h$ and $u$ are both continuous and $\cup_{n} X_{n}$ is dense in $X=(X, d)$. Thus $h_{n}$ pointwise converges to $u$ as $n \rightarrow \infty$, and $\mathcal{E}(u) \leq \lim _{n \rightarrow \infty} \mathcal{E}\left(h_{n}\right)<+\infty$, which implies that $\mathcal{E}(u)=\lim _{n \rightarrow \infty} \mathcal{E}\left(h_{n}\right)$. This proves Lemma 1.10. 
Lemma 1.11 In the case where $\mathcal{E}$ verifies [H-1], [H-2] and [H-4], taking a complete orthonormal system $\left\{\phi_{i}\right\}$ of the Hilbert space $D[\mathcal{E} ;\{o\}]$, where o is a fixed point of $X$, one has

$$
\begin{gathered}
g_{z}(x, y)=\sum_{i}\left(\phi_{i}(x)-\phi_{i}(z)\right)\left(\phi_{i}(y)-\phi_{i}(z)\right), \quad x, y, z \in X ; \\
R_{\mathcal{E}}(x, y)=\sum_{i}\left(\phi_{i}(x)-\phi_{i}(y)\right)^{2}, \quad x, y \in X .
\end{gathered}
$$

In the case where $\mathcal{E}$ verifies $[\mathrm{H}-5]$ and [H-2], taking a complete orthonormal system $\left\{\psi_{i}\right\}$ of $D[\mathcal{E}]$, one has

$$
g_{\mathcal{E}}(x, y)=\sum_{i} \psi_{i}(x) \psi_{i}(y), \quad x, y \in X .
$$

Proof. We consider the first case. For $v \in D[\mathcal{E} ;\{o\}]$, we have

$$
v=\sum_{i} \mathcal{E}\left(v, \phi_{i}\right) \phi_{i}
$$

in $D[\mathcal{E} ;\{o\}]$ and pointwise on $X$. If we fix two points $x, z \in X$, then we have

$$
\begin{aligned}
\mathcal{E} & \left(v, g_{z}(x, *)\right)=v(x)-v(z)=\sum_{i} \mathcal{E}\left(v, \phi_{i}\right) \phi_{i}(x)-\sum_{i} \mathcal{E}\left(v, \phi_{i}\right) \phi_{i}(z) \\
& =\sum_{i}\left(\phi_{i}(x)-\phi_{i}(z)\right) \mathcal{E}\left(v, \phi_{i}\right)=\sum_{i}\left(\phi_{i}(x)-\phi_{i}(z)\right) \mathcal{E}\left(v, \phi_{i}-\phi_{i}(z)\right) \\
& =\mathcal{E}\left(v, \sum_{i}\left(\phi_{i}(x)-\phi_{i}(z)\right)\left(\phi_{i}-\phi_{i}(z)\right)\right)
\end{aligned}
$$

Thus it holds that

$$
g_{z}(x, *)=\sum_{i}\left(\phi_{i}(x)-\phi_{i}(z)\right)\left(\phi_{i}(*)-\phi_{i}(z)\right)
$$

weakly in $D[\mathcal{E} ;\{o\}]$ and pointwise on $X$. Since $R_{\mathcal{E}}(x, z)=g_{z}(x, x)=$ $g_{x}(z, z)$, we get $R_{\mathcal{E}}(x, z)=\sum_{i}\left(\phi_{i}(x)-\phi_{i}(z)\right)^{2}$. The same arguments are valid for the second case. This completes the proof of Lemma 1.11.

In view of the above lemma, we obtain fundamental identities in the following

Theorem 1.12 In the case where $\mathcal{E}$ verifies [H-1], [H-2] and [H-4], one has

$$
\begin{aligned}
R_{\mathcal{E}}(x, y) & =g_{x}(y, y)=g_{y}(x, x)=g_{x}(y, z)+g_{y}(x, z), \\
g_{z}(x, y) & =\frac{1}{2}\left\{R_{\mathcal{E}}(x, z)+R_{\mathcal{E}}(z, y)-R_{\mathcal{E}}(x, y)\right\}
\end{aligned}
$$

for all $x, y, z \in X$. 
There are many important examples of Hilbert spaces possessing reproducing kernels. The following is used later (see Theorem 1.15).

Example 1.1 Consider the unit disk $\Omega=\{z=x+\sqrt{-1} y \in \mathbb{C}|| z \mid<1\}$ in the complex plane, and let $H D[\Omega]$ denote the Hilbert space of (real valued) harmonic functions $h$ on $\Omega$ with finite Dirichlet integrals $\mathcal{E}_{\Omega}(h)=$ $\iint_{\Omega}(\partial h / \partial x)^{2}+(\partial h / \partial y)^{2} d x d y$. Then the Green function can be computed explicitly as follows:

$$
g_{z}(p, q)=-\frac{1}{\pi} \log \left|1-\overline{\left(\frac{p-z}{1-\bar{p} z}\right)}\left(\frac{q-z}{1-\bar{q} z}\right)\right|, \quad p, q, z \in \Omega ;
$$

in particular, we have

$$
R_{\mathcal{E}_{\Omega}}(p, q)=-\frac{1}{\pi} \log \left(1-\left|\frac{p-q}{1-\bar{p} q}\right|^{2}\right), \quad p, q \in \Omega .
$$

We observe that the hyperbolic distance $\rho(p, q)$ between two points $p, q$ of $\Omega$ is given by

$$
\rho(p, q)=\log \left(1+\left|\frac{p-q}{1-\bar{p} q}\right|\right)-\log \left(1-\left|\frac{p-q}{1-\bar{p} q}\right|\right),
$$

and hence it holds that

$$
\rho(p, q)=\pi R_{\mathcal{E}_{\Omega}}(p, q)+2 \log \left(1+\left|\frac{p-q}{1-\bar{p} q}\right|\right) .
$$

Remark 1.1 (i) If a nonnegative quadratic form $\mathcal{E}$ satisfies [H-1], then the restriction $\mathcal{E}^{\prime}$ of $\mathcal{E}$ to $D[\mathcal{E} ; K]$ verifies $[\mathrm{H}-5]$ (with $M_{\mathcal{E}^{\prime}}(x)=g_{K}(x, x)$ ) for any nonempty subset $K$ of $X$.

(ii) Let $\mathcal{E}$ be a nonnegative quadratic form satisfying [H-5] and [H-2]. In the case where no nonzero constant functions belong to $D[\mathcal{E}]$, by letting $D[\hat{\mathcal{E}}]=\mathbb{R}+D[\mathcal{E}]$ and $\hat{\mathcal{E}}(c+u)=\mathcal{E}(u)$ for any $c \in \mathbb{R}$ and $u \in D[\mathcal{E}]$, we have a nonnegative quadratic form $\hat{\mathcal{E}}$ satisfying [H-1], [H-2] and [H-4]; the Green function $\hat{g}_{z}(x, y)(x, y, z \in X)$ is given by $g_{\mathcal{E}}(x, y)-g_{\mathcal{E}}(z, y)-$ $g_{\mathcal{E}}(x, z)+g_{\mathcal{E}}(z, z)$, and for $x, y \in X$, the number $R_{\hat{\mathcal{E}}}(x, y)=\sup \{\mid u(x)-$ $\left.\left.u(y)\right|^{2} / \hat{\mathcal{E}}(u) \mid u \in D[\hat{\mathcal{E}}], \hat{\mathcal{E}}(u)>0\right\}$ is equal to $g_{\mathcal{E}}(x, x)-2 g_{\mathcal{E}}(x, y)+g_{\mathcal{E}}(y, y)$.

(iii) Under condition [H-1] and [H-3], $\sup _{x, y \in X} R_{\mathcal{E}}(x, y)$ is bounded if and only if $D[\mathcal{E}]=B D[\mathcal{E}]$, that is, any $f \in D[\mathcal{E}]$ is bounded. In fact, it is evident that the former implies the latter, and it follows from the bounded inverse theorem that the latter implies the former. Similarly under condition [H-2] and [H-5], $M_{\mathcal{E}}$ is bounded on $X$ if and only if $D[\mathcal{E}]=B D[\mathcal{E}]$. See Proposition 4.1, Theorem 4.2 and Theorem 7.11 for related results. 
(iv) Let $(\mathcal{E}, D[\mathcal{E}])$ be a nonnegative quadratic form on a set $X$ satisfying [H-1] through [H-4]. Then $\sqrt{R_{\mathcal{E}}}$ induces a distance on $X$ and every function $u \in D[\mathcal{E}]$ uniquely extends to a continuous function $\hat{u}$ on the completion $\hat{X}$ of $(X, \sqrt{R})$. We define a quadratic form $\hat{\mathcal{E}}$ on $C(\hat{X})$ by letting $D[\hat{\mathcal{E}}]=$ $\{\hat{u} \mid u \in D[\mathcal{E}]\}$ and $\hat{\mathcal{E}}(\hat{u})=\mathcal{E}(u)$ for $\hat{u} \in D[\hat{\mathcal{E}}]$ and $\hat{\mathcal{E}}(\hat{u})=+\infty$ otherwise. It is evident that $(\hat{X}, \hat{\mathcal{E}})$ also satisfies $[\mathrm{H}-1]$ through $[\mathrm{H}-4]$.

1.2 In this part, we consider a finite set $X$ of cardinality $N$ and a nonnegative quadratic form $\mathcal{E}$ on $\ell(X)$.

By letting $c(x, y)=-\mathcal{E}\left(\chi_{x}, \chi_{y}\right)$ for $x, y \in X$ and $d(x)=-\sum_{y \in X} c(x, y)$ for $x \in X$, where $\chi_{x}$ stands for the characteristic function of a set $\{x\}$, the form is expressed as

$$
\begin{aligned}
\mathcal{E}(u, v) & =-\sum_{x, y \in X} c(x, y) u(x) v(y) \\
& =\frac{1}{2} \sum_{x, y \in X} c(x, y)\left(u(x)-u(y)(v(x)-v(y))+\sum_{x \in X} d(x) u(x) v(x),\right.
\end{aligned}
$$

for $u, v \in \ell(X)$. Given $u \in \ell(X)$, we set

$\mathcal{L}^{c} u(x)=-\sum_{y \in X} c(x, y) u(y)=\sum_{y \in X, y \neq x} c(x, y)(u(x)-u(y))+d(x) u(x), \quad x \in X$.

This is the self-adjoint operator associated to $\mathcal{E}$ relative to the counting measure $\mu^{c}$ on $X$.

Now we consider the case where $(\mathcal{E}, D[\mathcal{E}])$ satisfies $[\mathrm{H}-1]$ and $[\mathrm{H}-4]$; the latter is equivalent to the property: $d(x)=0$ for all $x \in X$. For a point $z \in X$, the definition of the Green functions $g_{z}$ reads

$$
\sum_{y \in X} g_{z}(x, y) c(y, w)=-\delta_{x w}
$$

for all $x \neq z$ and $w \neq z$, where $\delta_{x w}=1$ if $x=w$ and $\delta_{x w}=0$ otherwise. In other words, letting $(N-1) \times(N-1)$ matrices $C_{z}=(c(x, y))$ and $G_{z}=\left(g_{z}(x, y)\right)$ with $x, y \neq z$, we have $G_{z} C_{z}=I_{N-1}$ for $z \in X$, where $I_{N-1}$ stands for the unit matrix. By (1.3), we get

$$
\sum_{z, x, y \in X} g_{z}(x, y) c(x, y)=-N(N-1) .
$$

Moreover, recalling [H-1] saying that $\sum_{y \in X} c(x, y)=0$ for all $x$, we can derive from (1.2) and (1.4) the following identity:

$$
-\frac{1}{2} \sum_{x, y \in X} R_{\mathcal{E}}(x, y) \mathcal{E}\left(\chi_{x}, \chi_{y}\right)=N-1 .
$$


Now we mention a basic result derived from (1.1), (1.2) and (1.3) in the following

Theorem 1.13 Let $X$ be a finite set.

(i) For nonnegative quadratic forms $\mathcal{E}_{\alpha}(\alpha=1,2)$ on $\ell(X)$ satisfying $[\mathrm{H}-1]$ and $[\mathrm{H}-4], \mathcal{E}_{1}=\mathcal{E}_{2}$ if and only if $R_{\mathcal{E}_{1}}=R_{\mathcal{E}_{2}}$.

(ii) Let $K$ be a subset of $X$ and $\mathcal{E}$ (resp. $\mathcal{F}$ ) a nonnegative quadratic form on $\ell(X)$ (resp. $\ell(K)$ ) satisfying $[\mathrm{H}-1]$ and $[\mathrm{H}-4]$. Then $\mathcal{F}=\mathcal{E}_{K}^{*}$ if and only if $R_{\mathcal{F}}(x, y)=R_{\mathcal{E}}(x, y)$ for all $x, y \in K$.

The theorem corresponds to Theorem 2.1.12 and Corollary 2.1.13 in Kigami [32], where the Markov property [H-7] below is assumed.

Definition 1.2 Let $\mathcal{E}$ be a nonnegative quadratic form on a subspace $D[\mathcal{E}]$ of the space of functions, $\ell(X)$, on a set $X$.

(i) We say that $\mathcal{E}$ satisfies the Markov property if

$[\mathrm{H}-7]$ for any $u \in D[\mathcal{E}], \bar{u}=\max \{0, \min \{1, u\}\}$ also belongs to $D[\mathcal{E}]$ and satisfies $\mathcal{E}(\bar{u}) \leq \mathcal{E}(u)$.

(ii) A form $(\mathcal{E}, D[\mathcal{E}])$ on $X$ satisfying $[\mathrm{H}-1]$, [H-3], [H-4] and [H-7] is called a resistance form on $X$ and $R_{\mathcal{E}}(x, y)$ is called the effective resistance between points $x$ and $y \in X$.

In Kigami [32], a resistance form is required to satisfy the following additional condition: for any finite subset $V$ of $X$ and for any $u \in \ell(V)$, there exists $v \in D[\mathcal{E}]$ such that $v_{\mid V}=u$. However this is a consequence of the other conditions. In fact, the trace $\mathcal{E}_{K}^{*}$ on a subset $K$ of $X$ is also a resistance form on $K$, since $\mathcal{E}_{K}^{*}(u)=\mathcal{E}\left(H_{K ; u}\right) \geq \mathcal{E}\left(\overline{H_{K ; u}}\right) \geq \mathcal{E}\left(H_{K ; \bar{u}}\right)=\mathcal{E}_{K}^{*}(\bar{u})$; in particular, if $K$ is a finite subset, then $D\left[\mathcal{E}_{K}^{*}\right]=\ell(K)$, because $D\left[\mathcal{E}_{K}^{*}\right]$ is an algebra of unit element 1 in $\ell(K)$ separating points of $K$ (cf. Lemma 2.4).

Lemma 1.14 Let $(\mathcal{E}, D[\mathcal{E}])$ be a nonnegative quadratic form on $\ell(X)$. Suppose that $\chi_{x} \in D[\mathcal{E}]$ for all $x \in X$. Then $\mathcal{E}$ satisfies the Markov property $[\mathrm{H}-7]$ if and only if $\mathcal{E}\left(\chi_{x}, \chi_{y}\right) \leq 0$ for all $x, y \in X$ with $x \neq y$.

Proof. For all $x, y \in X$ with $x \neq y$, letting $u=\chi_{x}+t \chi_{y}$ with a constant $t<0$, we have $\bar{u}(=\max \{0, \min \{1, u\}\})=\chi_{x}$ and hence the Markov property implies that $\mathcal{E}\left(\chi_{x}, \chi_{x}\right)+2 t \mathcal{E}\left(\chi_{x}, \chi_{y}\right)+t^{2} \mathcal{E}\left(\chi_{y}, \chi_{y}\right) \geq \mathcal{E}\left(\chi_{x}, \chi_{x}\right)$; this is true for all $t<0$, and thus $\mathcal{E}\left(\chi_{x}, \chi_{y}\right) \leq 0$.

1.3 Let $G=(V, E)$ be a graph with the set of vertices $V$ and the set of edges $E$ that consists of pairs of vertices. In this paper, a graph admits no loops and multiple edges, and the set of vertices is finite or countably 
infinite. We say that a vertex $x$ is adjacent to another $y$ if $\{x, y\}$ belongs to $E$ and write $x \sim y$ to indicate it. We also use the notation: $|x y|$ for $\{x, y\} \in E$. For each vertex $x$, the cardinality of the subset $\{y \in V \mid y \sim x\}$ is called the degree of $G$ at $x$ and denoted by $\operatorname{deg}(x)$. When $\operatorname{deg}(x)$ is finite for each vertex $x$, we say that $G$ is locally finite. By a path of length $n$ in $G$, we mean a sequence of $(n+1)$ vertices $c=\left(x_{0}, x_{1}, \ldots, x_{n}\right)$ such that $x_{i} \sim x_{i+1}(i=0,1, \ldots, n-1)$, and we say that $c$ connects $x_{0}$ to $x_{n}$. $G$ is called a connected graph if for any pair of vertices $x$ and $y$, there exist paths connecting them. On a connected graph $G$, we can introduce a distance $d_{G}$ on $V$, called the graph distance of $G$, by assigning to each pair of vertices $x$ and $y$ the minimum of the length of a path connecting them. We are now given a weight $r$ on the set of edges $E$, that is a positive function on $E$ with the property that

$$
c(x)=\sum_{y \sim x} \frac{1}{r(|x y|)}<+\infty, \quad \forall x \in V .
$$

This is automatically satisfied in a locally finite graph. We call such a couple $(G, r)$ a network. Given a connected network $\Gamma=(V, E, r)$, a nonnegative quadratic form $\left(\mathcal{E}_{\Gamma}, D\left[\mathcal{E}_{\Gamma}\right]\right)$ on $\ell(V)$ can be defined as follows:

$$
D\left[\mathcal{E}_{\Gamma}\right]=\left\{u \in \ell(V) \mid \sum_{x \sim y} \frac{|u(x)-u(y)|^{2}}{r(|x y|)}<+\infty\right\} ;
$$

and

$$
\mathcal{E}_{\Gamma}(u)=\frac{1}{2} \sum_{x \sim y} \frac{|u(x)-u(y)|^{2}}{r(|x y|)}, \quad u \in D\left[\mathcal{E}_{\Gamma}\right] .
$$

A weight $r$ also gives rise to a distance $d_{r}$ on $V$ by taking $r(e)$ as the length of an edge $e$. To be precise, a path $c=\left(x_{0}, x_{1}, \ldots, x_{n}\right)$ has by definition the length $L_{r}(c)=\sum_{i=0}^{n-1} r\left(\left|x_{i} x_{i+1}\right|\right)$, and for any pair of vertices $x$ and $y$, $d_{r}(x, y)$ denotes the infimum of $L_{r}(c)$ over all paths $c$ joining $x$ and $y$. Then $d_{r}: V \times V \rightarrow[0,+\infty)$ is called the geodesic distance on $V$. For $r=1$, we write $d_{G}$ for $d_{1}$. For a pair of vertices $x$ and $y$, we connect $x$ to $y$ by a path $c=\left(x=x_{0}, x_{1}, \ldots, x_{n}=y\right)$. Then for a function $u \in D\left[\mathcal{E}_{\Gamma}\right]$, we have

$$
\begin{aligned}
|u(x)-u(y)| & \leq \sum_{i=0}^{n-1}\left|u\left(x_{i}\right)-u\left(x_{i+1}\right)\right| \\
& \leq\left(\sum_{i=0}^{n-1} \frac{\left|u\left(x_{i}\right)-u\left(x_{i+1}\right)\right|^{2}}{r\left(\left|x_{i} x_{i+1}\right|\right)}\right)^{1 / 2}\left(\sum_{i=0}^{n-1} r\left(\left|x_{i} x_{i+1}\right|\right)\right)^{1 / 2} \\
& \leq \mathcal{E}_{\Gamma}(u)^{1 / 2} L_{r}(c)^{1 / 2} .
\end{aligned}
$$


This holds for any path as above, and thus we get the following basic inequality:

$$
R_{\mathcal{E}_{\Gamma}}(x, y) \leq d_{r}(x, y), \quad x, y \in V .
$$

In this way, a resistance form on $V$ is provided by $\mathcal{E}_{\Gamma}$. The effective resistance and the reproducing kernel or the Green function of the form will be respectively denoted by $R_{\Gamma}$ and $g_{z}^{\Gamma}(x, y)(x, y, z \in V)$. Let $D_{0}\left[\mathcal{E}_{\Gamma}\right]$ be the closure of the set of finitely supported functions on $V$ in $D\left[\mathcal{E}_{\Gamma}\right]$ and $\mathcal{E}_{\Gamma}^{0}$ the restriction of $\mathcal{E}_{\Gamma}$ to $D_{0}\left[\mathcal{E}_{\Gamma}\right]$. Then the minimal effective resistance $R_{\Gamma}^{0}(x, y)$ between points $x, y \in V$ is introduced with respect to the form $\mathcal{E}_{\Gamma}^{0}$. It is evident from the definition that $R_{\Gamma}^{0} \leq R_{\Gamma}$. We recall here the fact (cf. [37], Theorem (3.63) and the references therein) that the following conditions are mutually equivalent: (i) $\mathcal{E}_{\Gamma}^{0}$ satisfies $[\mathrm{H}-5]$, (ii) $D_{0}\left[\mathcal{E}_{\Gamma}\right]$ contains no constant functions, (iii) $D_{0}\left[\mathcal{E}_{\Gamma}\right] \neq D\left[\mathcal{E}_{\Gamma}\right]$. If these are the cases, $D\left[\mathcal{E}_{\Gamma}\right]$ is decomposed into the direct sum of $D_{0}\left[\mathcal{E}_{\Gamma}\right]$ and the space $H D\left[\mathcal{E}_{\Gamma}\right]$ of harmonic functions of finite Dirichlet sums on $V$ that is the orthogonal complement of $D_{0}\left[\mathcal{E}_{\Gamma}\right]$ relative to the form; a function $h$ on $V$ belongs to $H D\left[\mathcal{E}_{\Gamma}^{0}\right]$ if and only if $h \in D\left[\mathcal{E}_{\Gamma}\right]$ and $\mathcal{L}^{c} h(x)=\sum_{y \sim x}(h(x)-h(y)) / r(|x y|)=0$ for all $x \in V$. We write $g_{\Gamma}^{0}(x, y)$ and $g_{z}^{0}(x, y)$ respectively for the Green functions of $\left(\mathcal{E}_{\Gamma}^{0}, D_{0}\left[\mathcal{E}_{\Gamma}\right]\right)$ and the extended form $\left(\hat{\mathcal{E}}_{\Gamma}^{0}, \mathbb{R}+D_{0}\left[\mathcal{E}_{\Gamma}\right]\right)$ as in Remark 1.1 (ii).

A locally finite graph $G=(V, E)$ is always assumed to be a network with weight 1.

Example 1.2 Let $T=(V, E)$ be a connected, locally finite tree and $r$ a weight on $E$. Then the effective resistance of the network $\Gamma=(T, r)$ coincides with the geodesic distance $d_{r}$ relative to $r$. When $r=1$ and $T$ is a homogeneous tree of degree $d \geq 3$, it is known that the minimal Green function of $\mathcal{E}_{T}^{0}$ is given by

$$
g_{T}^{0}(x, y)=\frac{d-1}{d(d-2)}\left(\frac{1}{d-1}\right)^{d_{T}(x, y)}, \quad x, y \in V
$$

(cf. [10]) and hence the minimal effective resistance of $T$ is given by

$$
R_{T}^{0}(x, y)=\frac{2(d-1)}{d(d-2)}\left(1-\left(\frac{1}{d-1}\right)^{d_{T}(x, y)}\right), \quad x, y \in V .
$$

Example 1.3 (cf. [13]) Let $G_{n}$ be a subgraph of the integer lattice $\mathbb{Z}^{d}=\left\{\left(x_{1}, \ldots, x_{d}\right) \mid x_{1}, \ldots, x_{n} \in \mathbb{Z}\right\}$ generated by the set of vertices $V_{n}=$ $\left\{\left(x_{1}, \ldots, x_{d}\right)|| x_{i} \mid \leq n, i=1, \ldots, d\right\}$. Then the effective resistance $R_{n}$ of $G_{n}$ satisfies

$$
c_{2} \log n \leq \max \left\{R_{n}(x, y) \mid x, y \in V_{n}\right\} \leq C_{2} \log n
$$


if $d=2$, and

$0<c_{d} \leq \min \left\{R_{n}(x, y) \mid x, y \in V_{n}, x \neq y\right\} \leq \max \left\{R_{n}(x, y) \mid x, y \in V_{n}\right\} \leq C_{d}$

if $d \geq 3$, where $c_{d}$ and $C_{d}$ are positive constants depending only on $d$.

Let $\Gamma=(V, E, r)$ be a connected, locally finite network. Regarding each edge $e$ as the segment $[0, r(e)]$ of length $r(e)$ and gluing the edges at the vertices, we obtain a Riemannian polyhedron $|\Gamma|$ of dimension one, which is locally compact and connected. In this paper, a Riemannian polyhedron of dimension one is called a geodesic graph for short. On the geodesic graph $|\Gamma|$, we have a canonical Riemannian measure $d s$ and the Riemannian distance $d_{r}$. The Dirichlet integral of a function $u \in C(|\Gamma|)$ is by definition

$$
\mathcal{E}_{|\Gamma|}(u)=\sum_{e \in E} \int_{0}^{r(e)}\left(\frac{d u}{d s}\right)^{2} d s
$$

if it is finite. Then $\left(\mathcal{E}_{|\Gamma|}, D\left[\mathcal{E}_{|\Gamma|}\right]\right)$ is a resistance form on $|\Gamma|$. Moreover the form is strong local in the sense that $\mathcal{E}_{|\Gamma|}(u, v)=0$ if $v$ is constant on the support of $u$. The energy measure $\mu_{\langle u\rangle}$ of a function $u$ of finite Dirichlet integral is defined by

$$
\begin{aligned}
\int_{|\Gamma|} \phi d \mu_{\langle u\rangle} & =\mathcal{E}_{|\Gamma|}(u, \phi u)-\frac{1}{2} \mathcal{E}_{|\Gamma|}\left(u^{2}, \phi\right) \\
& =\sum_{e \in E} \int_{0}^{r(e)} \phi\left(\frac{d u}{d s}\right)^{2} d s, \quad \phi \in C(|\Gamma|) .
\end{aligned}
$$

For functions $u, v \in D\left[\mathcal{E}_{|\Gamma|}\right]$, we have the signed Radon measure defined by $\mu_{\langle u, v\rangle}=\left(\mu_{\langle u+v\rangle}-\mu_{\langle u\rangle}-\mu_{\langle v\rangle}\right) / 2$. This means in this case that

$$
\int_{|\Gamma|} \phi d \mu_{\langle u, v\rangle}=\sum_{e \in E} \int_{0}^{r(e)} \phi \frac{d u}{d s} \frac{d v}{d s} d s, \quad \phi \in C(|\Gamma|) .
$$

The resistance form $\mathcal{E}_{\Gamma}$ of $\Gamma$ may be considered as the trace of $\mathcal{E}_{|\Gamma|}$ on $V$. A function $u \in D\left[\mathcal{E}_{\Gamma}\right]$ is identified with a function $\tilde{u} \in D\left[\mathcal{E}_{|\Gamma|}\right]$ which is linear on each edge and equal to $u$ on $V$. Moreover it holds that $\mathcal{E}_{|\Gamma|}(\tilde{u}, \phi)=\mathcal{E}_{\Gamma}\left(u, \phi_{\mid V}\right)$ for $u \in D\left[\mathcal{E}_{\Gamma}\right]$ and $\phi \in D\left[\mathcal{E}_{|\Gamma|}\right]$; in particular, the Dirichlet sum of $u$ is equal to the Dirichlet integral of $\tilde{u}$.

Now we consider a nonnegative Markovian form $\mathcal{E}$ on a finite set $X$ such that $\chi_{x} \in D[\mathcal{E}]$ for all $x \in V$. A point of $X$ is called a vertex, and a vertex $x$ is, by definition, adjacent to another $y$ if $c(x, y):=-\mathcal{E}\left(\chi_{x}, \chi_{y}\right)>0$. If this is the case, $x$ and $y$ are assumed to be joined by an edge of resistance 
$r(|x y|)=c(x, y)^{-1}$. In this way, we get a finite (resistive) network $(X, E, r)$, where $E$ stands for the set of edges, each of which is assigned the positive number $r$. It is easy to see that if $[\mathrm{H}-1]$ holds, then the graph $(X, E)$ is connected, and conversely, if the graph is connected and $d(x)=\mathcal{E}\left(\chi_{x}, 1\right) \geq 0$ for all $x \in X$, then [H-1] is verified.

Now in view of Example 1.1, we can prove the following

Theorem 1.15 Let $G=(V, E)$ be a connected, locally finite, infinite graph uniformly embedded in the unit disk $\Omega$ in $\mathbb{C}$ endowed with the hyperbolic distance $\rho$, that is, $V \subset \Omega$ and there is a positive constant $c$ such that

$$
c^{-1} \rho(x, y) \leq d_{G}(x, y) \leq c \rho(x, y), \quad x, y \in V .
$$

Then there exist positive constants $c^{\prime}$ and $c^{\prime \prime}$ such that

$$
c^{\prime}\left(d_{G}(x, y)-c^{\prime \prime}\right) \leq R_{G}(x, y) \leq c d_{G}(x, y), \quad x, y \in V .
$$

Proof. In what follows, $c_{i}$ 's stand for positive constants depending only on $c$ as above and $\Omega$ is endowed with the Poincaré metric $g_{P}$.

We first notice that for any $x \in V$, the degree at $x$, $\operatorname{deg}(x)$, and the number of the vertices $y$ such that $B_{c_{1}}(x) \cap B_{c_{1}}(y) \neq \emptyset$ are bounded from above by $c_{2}$, where $B_{a}(x)$ denotes the metric ball of $\Omega$ around a point $x$ with radius $a$ (cf. [11], [37, Chap. IV $\S 6]$ ).

Secondly we observe that a function $h \in H D[\Omega]$ satisfies

$$
|h(x)-h(y)|^{2} \leq c_{3} \int_{B_{c_{4}}(x)}|d h|_{g_{P}}^{2} d v_{g_{P}}
$$

for all $x, y \in V$ with $x \sim y$. Therefore we have

$$
\begin{aligned}
\mathcal{E}_{G}\left(h_{\mid V}\right) & =\frac{1}{2} \sum_{x \in V} \sum_{y \sim x}|h(x)-h(y)|^{2} \leq c_{3} \sum_{x \in V} \operatorname{deg}(x) \int_{B_{c_{4}}(x)}|d h|_{g_{P}}^{2} d v_{g_{P}} \\
& \leq c_{5} \int_{\Omega}|d h|_{g_{P}}^{2} d v_{g_{P}}=c_{5} \mathcal{E}_{\Omega}(h) .
\end{aligned}
$$

This shows that

$$
\frac{|h(x)-h(y)|^{2}}{\mathcal{E}_{\Omega}(h)} \leq c_{5} \frac{|h(x)-h(y)|^{2}}{\mathcal{E}_{G}\left(h_{\mid V}\right)} \leq c_{5} R_{G}(x, y)
$$

for all $x, y \in V$. Now given $x, y \in V$, letting $h=g_{x}(y, *)$, where $g_{x}(y, z)$ denotes the Green function of $\mathcal{E}_{\Omega}$ exhibited in Example 1.1, we have

$$
R_{\mathcal{E}_{\Omega}}(x, y) \leq c_{5} R_{G}(x, y), \quad x, y \in V .
$$

In view of the relation between $R_{\mathcal{E}_{\Omega}}$ and the hyperbolic distance described in Example 1.1, we arrive at the required estimate. This completes the proof of the theorem. 
Remark 1.2 Suppose $\left(X, d_{X}\right)$ and $\left(Y, d_{Y}\right)$ are metric spaces. A map $f$ : $X \rightarrow Y$ is called a quasi-isometric embedding of $X$ into $Y$ if there exist constants $\lambda \geq 1$ and $k \geq 0$ such that

$$
\frac{1}{\lambda} d_{X}\left(x, x^{\prime}\right)-k \leq d_{Y}\left(f(x), f\left(x^{\prime}\right)\right) \leq \lambda d_{X}\left(x, x^{\prime}\right)+k
$$

for all $x, x^{\prime} \in X$. If in addition, for each $y \in Y$, there exists $x \in X$ such that $d_{Y}(f(x), y) \leq k$, then $f$ is called a quasi-isometry (or a rough isometry). Let $G=(V, E)$ be a connected, locally finite, infinite graph of bounded degrees and suppose that it is quasi-isometric embedded in the unit disk $\Omega$ in $\mathbb{C}$ endowed with the hyperbolic distance $\rho$. Then the same conclusion of Theorem 1.15 holds.

\section{Resistance forms}

In this section, we prove some fundamental results on resistance forms, the maximum principle, Harnack's inequality, Caccioppoli's inequality and so on.

2.1 Let $\Gamma=(V, E, r)$ be a connected, finite network. Given a subset $K$ of $V, H \in \ell(V)$ satisfies $\mathcal{E}_{\Gamma}(H, v)=0$ for all functions $v$ vanishing on $K$ if and only if $H$ satisfies $\mathcal{L}^{c} H(x)=\sum_{y \sim x} c(x, y)(H(x)-H(y))=0$ for any $x \in X \backslash K$, where we put $c(x, y)=r(|x y|)^{-1}$. Such a function $H$ is said to be harmonic on $X \backslash K$. The nonnegativity of $c(x, y)$ verifies the maximum principle for harmonic functions as follows:

Lemma 2.1 Let $\Gamma=(V, E, r)$ be as above and $K$ a subset of $V$. Let $H$ be a function on $V$ that is harmonic on $V \backslash K$. Then one has

$$
\min _{a \in K} H(a) \leq H(x) \leq \max _{a \in K} H(a), \quad x \in V
$$

The maximum principle applied to the Green functions $g_{z}(x, y)$ yields the fact that $0 \leq g_{z}(x, y) \leq g_{z}(x, x)$ for all $x, y, z \in V$. Therefore in view of identity (1.2), the effective resistance $R_{\Gamma}$ of $\Gamma$ satisfies the triangle inequality (cf. [13], [38]), and thus $R_{\Gamma}$ provides a distance on $V$, called the resistance metric of $\Gamma$ in $[32]$.

For a subset $A$ of $V$, we denote by $b A$ the subset of $A$ consisting of the vertices adjacent to some of $V \backslash A$. Let $K$ and $A$ be subsets of $V$ such that $K \cap A=\emptyset$. Given a function $H$ on $X$ that is positive on $(V \backslash K) \cup b K$ and harmonic on $V \backslash K$, we consider a unique solution $P_{H}$ on $V$ of equation: $\mathcal{L}^{c} P_{H}=0$ in $V \backslash(K \cup b A)$, subject to the boundary condition: $P_{H}=0$ on $K$ 
and $P_{H}=H$ on $b A$. Then the maximum principle verifies that $P_{H}=H$ on $A, P_{H} \leq H$ on $(V \backslash K) \cup b K$, and hence $\mathcal{L}^{c} P_{H} \geq 0$ outside of $K$. Note that for any $x \in A$, we have

$$
H(x)=P_{H}(x)=\mathcal{E}\left(P_{H}, g_{K}(x, *)\right)=\sum_{y \in b A} g_{K}(x, y) \mathcal{L}^{c} P_{H}(y) .
$$

Let

$$
C(V \backslash K, A)=\max \left\{\frac{g_{K}(x, z)}{g_{K}(y, z)} \mid x, y \in A, z \in b A\right\} .
$$

Then we have

$$
\begin{aligned}
H(x) & =\sum_{z \in b A} \frac{g_{K}(x, z)}{g_{K}(y, z)} g_{K}(y, z) \mathcal{L}^{c} P_{H}(z) \\
& \leq C(V \backslash K, A) \sum_{z \in b A} g_{K}(y, z) \mathcal{L}^{c} P_{H}(z) \\
& =C(V \backslash K, A) H(y)
\end{aligned}
$$

for all $x, y \in A$. Thus we arrive at Harnack's inequality in the following

Lemma 2.2 Let $\Gamma=(V, E, r)$ be a connected, finite network. Let $K$ and $A$ be subsets of $V$ such that $K \cap A=\emptyset$. Then one has

$$
\max _{x \in A} H \leq C(V \backslash K, A) \min _{x \in A} H
$$

for any $H \in \ell(V)$ that is positive on $(V \backslash K) \cup b K$ and harmonic on $V \backslash K$.

Now we prove a version of the Caccioppoli inequality on $\Gamma$.

Lemma 2.3 Let $\Gamma=(V, E, r)$ be as above and $K$ a subset of $V$. Let $H$ be a function on $V$ that is harmonic on $V \backslash K$. Then for any $\xi \in \ell(V)$ with $\xi=0$ on $K$, one has

$$
\mathcal{E}_{\Gamma}(\xi H) \leq 10 \sup _{V}|H|^{2} \mathcal{E}_{\Gamma}(\xi)
$$

Proof. Let $|\Gamma|$ be the metric graph associated to $\Gamma$. For $u \in \ell(V)$, we denote by $\tilde{u}$ the harmonic extension of $u$ to $|\Gamma|$, namely the function on $|\Gamma|$ which is linear on the segment assigned to each edge of $\Gamma$ and coincides with $u$ on $V$. Then we have

$$
\begin{aligned}
\mathcal{E}_{|\Gamma|}\left((\xi H)^{\sim}\right) & \leq \mathcal{E}_{|\Gamma|}(\tilde{\xi} \tilde{H})=\int \tilde{H}^{2} d \mu_{\langle\tilde{\xi}\rangle}+2 \int \tilde{\xi} \tilde{H} d \mu_{\langle\tilde{\xi}, \tilde{H}\rangle}+\int \tilde{\xi}^{2} d \mu_{\langle\tilde{H}\rangle} \\
& \leq 2\left\{\int \tilde{H}^{2} d \mu_{\langle\tilde{\xi}\rangle}+\int \tilde{\xi}^{2} d \mu_{\langle\tilde{H}\rangle}\right\} .
\end{aligned}
$$


Since $\mathcal{E}_{|\Gamma|}\left(\tilde{\xi}^{2} \tilde{H}, \tilde{H}\right)=\mathcal{E}_{\Gamma}\left(\xi^{2} H, H\right)=0$ and

$$
\mathcal{E}_{|\Gamma|}\left(\tilde{\xi}^{2} \tilde{H}, \tilde{H}\right)=\int \tilde{\xi}^{2} d \mu_{\langle\tilde{H}\rangle}+2 \int \tilde{\xi} \tilde{H} d \mu_{\langle\tilde{\xi}, \tilde{H}\rangle} \geq \frac{1}{2} \int \tilde{\xi}^{2} d \mu_{\langle\tilde{H}\rangle}-2 \int \tilde{H}^{2} d \mu_{\langle\tilde{\xi}\rangle},
$$

we obtain

$$
\int \tilde{\xi}^{2} d \mu_{\langle\tilde{H}\rangle} \leq 4 \int \tilde{H}^{2} d \mu_{\langle\tilde{\xi}\rangle}
$$

Therefore we get

$$
\mathcal{E}_{|\Gamma|}\left((\xi H)^{\sim}\right) \leq 10 \int \tilde{H}^{2} d \mu_{\langle\tilde{\xi}\rangle} \leq 10 \sup \left\{\tilde{H}(x)^{2} \mid x \in \operatorname{supp} \mu_{\langle\tilde{\xi}\rangle}\right\} \mathcal{E}_{|\Gamma|}(\tilde{\xi}) .
$$

This shows that

$$
\mathcal{E}_{\Gamma}(\xi H) \leq 10 \sup _{V}|H|^{2} \mathcal{E}_{\Gamma}(\xi) .
$$

Thus the proof of Lemma 2.3 is completed.

Finally we recall two basic properties of connected finite networks.

Lemma 2.4 Let $\Gamma=(V, E, r)$ be as above. Then for all $u, v \in \ell(V)$, one has

$$
\mathcal{E}_{\Gamma}(u v)^{1 / 2} \leq \sup |u| \mathcal{E}_{\Gamma}(v)^{1 / 2}+\sup |v| \mathcal{E}_{\Gamma}(u)^{1 / 2} .
$$

Lemma 2.5 (Rayleigh's monotonicity principle) Let $\Gamma=(V, E, r)$ be as above and $\Gamma^{\prime}=\left(V^{\prime}, E^{\prime}, r^{\prime}\right)$ a connected subnetwork, that is, $V^{\prime} \subset V$, $E^{\prime} \subset E$ and $r^{\prime}=r$ on $E^{\prime}$. Then

$$
R_{\Gamma}(x, y) \leq R_{\Gamma^{\prime}}(x, y) \quad \text { for all } x, y \in X^{\prime} .
$$

Remark 2.1 For a finite, connected, network $\Gamma=(V, E, r)$ of $N$ vertices, the identity (1.5) reads

$$
\frac{1}{2} \sum_{x \sim y} \frac{R_{\Gamma}(x, y)}{r(|x y|)}=N-1 .
$$

This is a classical result due to Foster (cf. [20], [37], [38]).

2.2 In this part, we consider a resistance form $\mathcal{E}$ on a set $X$, where [H-6] is assumed. To begin with, applying the results of the previous sections, we can deduce the following

Proposition 2.6 For a resistance form $\mathcal{E}$ on a set $X$, it holds that

$$
0 \leq g_{z}(x, y) \leq g_{z}(x, x), \quad x, y, z \in X
$$


Now the following is an immediate consequence from Lemma 2.1.

Theorem 2.7 Let $K$ be a closed subset of $X$. Let $H$ be a bounded function in $D[\mathcal{E}]$ that satisfies $\mathcal{E}(H, v)=0$ for all $v \in D[\mathcal{E} ; K]$. Then one has

$$
\inf _{K} H \leq H(x) \leq \sup _{K} H, \quad x \in X
$$

In view of Lemma 2.2, we can deduce the following

Theorem 2.8 Let $K$ be a closed subset of $X$. If a positive function $H \in D[\mathcal{E}]$ satisfies $\mathcal{E}(H, v)=0$ for all $v \in D[\mathcal{E} ; K]$, then for a compact subset $A$ of $X \backslash K$, one has

$$
H(x) \leq C(X \backslash K, A) H(y)
$$

for all $x, y \in A$, where

$$
C(X \backslash K, A)=\sup \left\{\frac{g_{K}(x, z)}{g_{K}(y, z)} \mid x, y, z \in A\right\} .
$$

In the following lemma, we prove a version of Caccioppoli inequality on $X$.

Theorem 2.9 Let $K$ be a closed subset of $X$. Let $H$ be a bounded function in $D[\mathcal{E}]$ such that $\mathcal{E}(H, v)=0$ for all $v \in D[\mathcal{E} ; K]$. Then one has

$$
\mathcal{E}(\xi H) \leq 10 \sup _{X}|H|^{2} \mathcal{E}(\xi)
$$

for all $\xi \in D[\mathcal{E} ; K]$.

Proof. We first take an increasing sequence of finite subsets $X_{n}$ of $X$ in such a way that $\cup_{n} X_{n}$ and $K \cap\left(\cup_{n} X_{n}\right)$ are dense in $X$ and $K$, respectively. Let $H_{n}$ be a unique function in $D[\mathcal{E}]$ such that $H_{n}=H$ on $K_{n}=K \cap X_{n}$ and $\mathcal{E}(H, v)=0$ for all $v \in D\left[\mathcal{E} ; K_{n}\right]$. Then as $n \rightarrow \infty, \mathcal{E}\left(H_{n}\right)$ tends to $\mathcal{E}(H)$ and $H_{n}$ converges to $H$ pointwise in $X$. Let $h_{n}$ (resp. $\xi_{n}$ ) denote the restriction of $H_{n}$ (resp. $\xi$ ) to $X_{n}$. Then $\mathcal{E}_{X_{n}}^{*}\left(h_{n}, v\right)=0$ for all $v \in D\left[\mathcal{E}_{X_{n}}^{*} ; K_{n}\right]$. Therefore we are allowed to apply Lemma 2.3 to $h_{n}$ and $\xi_{n}$, obtaining

$$
\mathcal{E}_{X_{n}}^{*}\left(\xi_{n} h_{n}\right) \leq 10 \sup _{X_{n}}\left|h_{n}\right|^{2} \mathcal{E}_{X_{n}}^{*}\left(\xi_{n}\right) .
$$

Let $F_{n}=H_{X_{n} ; \xi_{n} h_{n}}$ for simplicity. Then $F_{n}$ converges to $\xi H$ pointwise in $\cup_{n} X_{n}$ and hence in $X$, because $\mathcal{E}\left(F_{n}\right)\left(=\mathcal{E}_{X_{n}}^{*}\left(\xi_{n} H_{n}\right)\right)$ is bounded and $\cup_{n} X_{n}$ is dense in $X$. Therefore we have

$$
\mathcal{E}(\xi H) \leq \liminf _{n \rightarrow \infty} \mathcal{E}\left(F_{n}\right)=\liminf _{n \rightarrow \infty} \mathcal{E}_{X_{n}}^{*}\left(\xi_{n} h_{n}\right) \leq 10 \sup |H|^{2} \mathcal{E}(\xi) .
$$

This completes the proof of Theorem 2.9. 
In what follows, we assume further that the metric space $(X, d)$ is compact. Then $\mathcal{E}$ is a regular Dirichlet form on $X$ defined on the $L^{2}$-space relative to a positive Radon measure on $X$ with support $X$ (cf. e.g. [21]). Recall first that given a function $u \in D[\mathcal{E}]$, a Radon measure $\mu_{\langle u\rangle}$ on $X$, called the energy measure of $u$, is defined by $\int \phi d \mu_{\langle u\rangle}=\mathcal{E}(u, \phi u)-\frac{1}{2} \mathcal{E}\left(u^{2}, \phi\right)$ for $\phi \in D[\mathcal{E}]$. We note here that $D[\mathcal{E}]$ is dense in $C(X, d)$. Now given a proper open set $\Omega$ of $X$ and a closed subset $A$ of $\Omega$, we let

$$
\operatorname{Cap}_{\Omega}(A)=\inf \{\mathcal{E}(u) \mid u \in D[\mathcal{E} ; X \backslash \Omega], u \geq 1 \text { on } A\} .
$$

This is called the capacity of $A$ in $\Omega$. We recall that there exists a unique function $e_{\Omega, A} \in D[\mathcal{E} ; X \backslash \Omega]$ such that $e_{\Omega, A} \geq 1$ on $A$ and $\mathcal{E}\left(e_{\Omega, A}\right)=\operatorname{Cap}_{\Omega}(A) ;$ the function is called the equilibrium potential of the subset $A$ of $\Omega$ and characterized as a function such that $e_{\Omega, A}=1$ on $A$ and $\mathcal{E}\left(e_{\Omega, A}, v\right) \geq 0$ for all $v \in D[\mathcal{E} ; X \backslash \Omega]$ that are nonnegative on $A$ (cf. ibid. Chap.2). The equilibrium potential is expressed as $e_{\Omega, A}(x)=\int g_{X \backslash \Omega}(x, y) d \nu(y)$ for $x \in X$, where $\nu$ is a positive Radon measure on $X$ supported in $A$. Observing that $\mathcal{E}\left(g_{X \backslash \Omega}(z, *) / \min _{x, y \in A} g_{X \backslash \Omega}(x, y)\right)=g_{X \backslash \Omega}(z, z) /\left(\min _{x, y \in A} g_{X \backslash \Omega}(x, y)\right)^{2}$, we have the following estimate for the capacity of $A$ :

$$
\operatorname{Cap}_{\Omega}(A) \leq \frac{\min _{x \in A} g_{X \backslash \Omega}(x, x)}{\left(\min _{x, y \in A} g_{X \backslash \Omega}(x, y)\right)^{2}} .
$$

Now we assume further that $\mathcal{E}$ is local in the sense that $\mathcal{E}(u, v)=0$ if $\operatorname{supp} u \cap \operatorname{supp} v=\emptyset$. Let us denote by $D_{\Omega, l o c}[\mathcal{E}]$ the space of functions $u$ on $\Omega$ such that for any relatively compact open subset $\omega$ of $\Omega$, there exist functions $v \in D[\mathcal{E}]$ that coincide with $u$ on $\omega$. We say a function $H$ on $\Omega$ is $\mathcal{E}$-harmonic if $H \in D_{\Omega, l o c}[\mathcal{E}]$ and $\mathcal{E}(H, v)=0$ for all $v \in D[\mathcal{E}]$ supported in $\Omega$. Then Theorems 2.7 and 2.8 extend to the following

Theorem 2.10 Let $X, \Omega$ and $\mathcal{E}$ be as above. Let $H$ be an $\mathcal{E}$-harmonic in $\Omega$.

(i) When $H$ is bounded, one has

$$
\inf _{X \backslash \Omega} H \leq H(x) \leq \sup _{X \backslash \Omega} H, \quad x \in \Omega,
$$

where one set

$$
\begin{aligned}
& \inf _{X \backslash \Omega} H=\lim _{\delta \rightarrow 0} \inf \{H(x) \mid x \in \Omega, d(x, X \backslash \Omega)<\delta\} ; \\
& \sup _{X \backslash \Omega} H=\lim _{\delta \rightarrow 0} \sup \{H(x) \mid x \in \Omega, d(x, X \backslash \Omega)<\delta\} .
\end{aligned}
$$

(ii) When $H$ is positive, then given a closed subset $A$ of $\Omega$, one has

$$
H(x) \leq C(\Omega, A) H(y)
$$

for all $x, y \in A$. 
Now we prove the following

Theorem 2.11 Let $X, \Omega$ and $\mathcal{E}$ be as above. Then for any function $f \in$ $C(X \backslash \Omega)$, there exists uniquely a function $H \in C(X) \cap D_{\Omega, l o c}[\mathcal{E}]$ such that $H$ is equal to $f$ on $X \backslash \Omega$ and $\mathcal{E}$-harmonic in $\Omega$.

Proof. Let $K=X \backslash \Omega$ and note that $D\left[\mathcal{E}_{K}^{*}\right]$ is dense in $C(K)$. Let $\left\{f_{n}\right\}$ be a sequence in $D\left[\mathcal{E}_{K}^{*}\right]$ which uniformly converges to a given $f \in C(K)$, and let $H_{n}=H_{K ; f_{n}} \in D[\mathcal{E}]$. Then in view of Theorem 2.10, $\left\{H_{n}\right\}$ is a Cauchy sequence in $C(X)$ and we let $H=\lim _{n \rightarrow \infty} H_{n}$. Then it follows from Theorem 2.9 that $\mathcal{E}\left(e_{\Omega, A} H_{n}\right) \leq 10 \sup _{X}\left|H_{n}\right|^{2} \operatorname{Cap}_{\Omega}(A)$ for any closed subset $A$ of $\Omega$ and each $n$. Therefore we have

$$
\mathcal{E}\left(e_{\Omega, A} H\right) \leq \liminf _{n \rightarrow \infty} \mathcal{E}\left(e_{\Omega, A} H_{n}\right) \leq 10 \sup _{K}|f|^{2} \operatorname{Cap}_{\Omega}(A)<+\infty .
$$

Thus $H$ belongs to $D_{\Omega, l o c}[\mathcal{E}]$ and $\mathcal{E}\left(e_{\Omega, A} H, v\right)=\lim _{n \rightarrow \infty} \mathcal{E}\left(e_{\Omega, A} H_{n}, v\right)$ for any $v \in D[\mathcal{E}]$ supported in $\Omega$. Now choosing an open subset $\omega$ in such a way that supp $v \subset \omega \subset \bar{\omega} \subset \Omega$ and considering the locality of the form, we obtain $\mathcal{E}(H, v)=\mathcal{E}\left(e_{\Omega, \bar{\omega}} H, v\right)=\lim _{n \rightarrow \infty} \mathcal{E}\left(e_{\Omega, \bar{\omega}} H_{n}, v\right)=\lim _{n \rightarrow \infty} \mathcal{E}\left(H_{n}, v\right)=0$. This completes the proof of Theorem 2.11.

\section{Laplace operators}

In this section, we introduce a certain measure on a space endowed with a resistance form and carry out embedding the space into the Hilbert space of square summable sequences, using the eigenvalues and eigenfunctions of the self-adjoint operator to get a regular Dirichlet space containing the space as a dense subset.

3.1 We consider a quadratic form $\mathcal{E}$ on a set $X$ satisfying [H-1], [H-2], [H-4] and [H-6]. Let $\mu$ be a Borel measure on $X=(X, d)$ and $K$ the support of $\mu$, supp $\mu$, that is the complement of the union of open balls $B_{r}(p)$ with $\mu\left(B_{r}(p)\right)=0$. Note that continuous functions $f$ and $g$ coincide almost everywhere in $X$ if and only if $f=g$ on $K$. Suppose that

$$
\mu(X)<+\infty ; \quad \int_{X} R_{\mathcal{E}}(o, x) d \mu(x)<+\infty .
$$

Since any function $u \in D[\mathcal{E}]$ satisfies $|u(x)|^{2} \leq 2 \mathcal{E}(u) R_{\mathcal{E}}(x, y)+2 u(y)^{2}$ for all $x, y \in X$, it belongs to $L^{2}(K, \mu)$, and moreover, noting that $\sqrt{R_{\mathcal{E}}}$ satisfies the triangle inequality, we have

$$
\begin{aligned}
|u(x)|^{2} & \leq 2 \mathcal{E}(u) \int_{X} R_{\mathcal{E}}(x, y) d \mu(y)+2 \int_{X} u(y)^{2} d \mu(y) \\
& \leq 4 \mathcal{E}(u)\left(\int_{X} R_{\mathcal{E}}(o, y) d \mu(y)+R_{\mathcal{E}}(o, x) \mu(X)\right)+2 \int_{X} u(y)^{2} d \mu(y)
\end{aligned}
$$


for all $x \in X$. This shows that $u^{2}$ is bounded from above by an integrable function on $X$ depending only on $\mathcal{E}(u), \int_{X} u^{2} d \mu$ and $\mu(X)$. Therefore any sequence of functions $u_{n} \in D[\mathcal{E}]$ with $\sup _{n}\left(\mathcal{E}\left(u_{n}\right)+\int_{X} u_{n}^{2} d \mu\right)<\infty$ contains a subsequence that converges to a function pointwise in $X$ and hence in $L^{2}(K, \mu)$ because of Lebesgue's convergence theorem. Thus the embedding of $D\left[\mathcal{E}_{K}^{*}\right]$ into $L^{2}(K, \mu)$ is compact (cf. $\left.[32,2.4]\right)$.

Now we denote by $\mathcal{L}_{\mu}$ the self-adjoint operator associated with the closed form $\mathcal{E}_{K}^{*}$ on the $L^{2}$-closure of $D\left[\mathcal{E}_{K}^{*}\right]$; the domain $D\left[\mathcal{L}_{\mu}\right]$ of $\mathcal{L}_{\mu}$ consists of functions $u \in D\left[\mathcal{E}_{K}^{*}\right]$ such that the functional on $D\left[\mathcal{E}_{K}^{*}\right]$ defined by $v \rightarrow$ $\mathcal{E}_{K}^{*}(u, v)$ is continuous with respect to the $L^{2}$-norm, and for any $u \in D\left[\mathcal{L}_{\mu}\right]$, $\mathcal{L}_{\mu} u$ is the unique function (in the closure of $D\left[\mathcal{E}_{K}^{*}\right]$ in $L^{2}(K, \mu)$ ) satisfying

$$
\mathcal{E}_{K}^{*}(v, u)=\int_{K} v \mathcal{L}_{\mu} u d \mu, \quad v \in D\left[\mathcal{E}_{K}^{*}\right] .
$$

Thus we have the following

Lemma 3.1 Under condition (3.1), the embedding of $D\left[\mathcal{E}_{K}^{*}\right]$ into $L^{2}(K, \mu)$ $(K=\operatorname{supp} \mu)$ is compact, and the spectra of the operator $\mathcal{L}_{\mu}$ consists of a nondecreasing sequence of nonnegative numbers $\left\{\lambda_{i}(\mu)\right\}$ that diverges to infinity as $i \rightarrow \infty$ unless $D\left[\mathcal{E}_{K}^{*}\right]$ is of finite dimension.

Let $N=\operatorname{dim} D\left[\mathcal{E}_{K}^{*}\right] \leq+\infty$ and $\left\{\phi_{i}: 0 \leq i \leq N-1\right\}$ a complete orthonormal system of eigenfunctions $\phi_{i}$ with eigenvalues $\lambda_{i}\left(\mathcal{E}_{K}^{*} ; \mu\right)$ in $L^{2}(K, \mu)$, where $\phi_{0}=1 / \sqrt{\mu(K)}$. We write $\lambda_{i}(\mu)$ for $\lambda_{i}\left(\mathcal{E}_{K}^{*} ; \mu\right)$ if there is no confusion. In view of Lemma 1.11, $g_{z}(x, y)$ and $R_{\mathcal{E}}(x, y)$ can be expressed as follows:

$$
\begin{aligned}
g_{z}(x, y) & =\sum_{i=1}^{N-1} \frac{1}{\lambda_{i}(\mu)}\left(\phi_{i}(x)-\phi_{i}(z)\right)\left(\phi_{i}(y)-\phi_{i}(z)\right), \\
R_{\mathcal{E}}(x, y) & =\sum_{i=1}^{N-1} \frac{1}{\lambda_{i}(\mu)}\left(\phi_{i}(x)-\phi_{i}(y)\right)^{2} .
\end{aligned}
$$

Fix $x, y \in K$. Then the function $g_{z}(x, y)$ of $z \in X$ is summable over $K$, because of identity (1.2). We set

$$
g_{\mu}(x, y):=\frac{1}{\mu(K)} \int_{K} g_{z}(x, y) d \mu(z)
$$

Then in view of (3.2) and (3.3), the following identities hold:

$$
\begin{aligned}
g_{\mu}(x, y) & =\frac{1}{\mu(K)}\left(\sum_{i=1}^{N-1} \frac{1}{\lambda_{i}(\mu)}\right)+\sum_{i=1}^{N} \frac{\phi_{i}(x) \phi_{i}(y)}{\lambda_{i}(\mu)} \\
\int_{K} g_{\mu}(x, y) d \mu(y) & =\frac{1}{2 \mu(K)} \iint_{K \times K} R_{\mathcal{E}}(y, z) d \mu(y) d \mu(z)=\sum_{i=1}^{N-1} \frac{1}{\lambda_{i}(\mu)} .
\end{aligned}
$$


The last identity yields a lower bound for $\lambda_{i}(\mu)(i=1,2, \ldots, N-1)$ as follows:

$$
\lambda_{i}(\mu) \geq \frac{2 \mu(K) i}{\iint_{K \times K} R_{\mathcal{E}}(y, z) d \mu(y) d \mu(z)}
$$

We put

$$
g_{\mu}^{*}(x, y):=g_{\mu}(x, y)-\frac{1}{\mu(K)} \sum_{i=1}^{N-1} \frac{1}{\lambda_{i}(\mu)}=\sum_{i=1}^{N-1} \frac{\phi_{i}(x) \phi_{i}(y)}{\lambda_{i}(\mu)}, \quad x, y \in K
$$

Notice that

$$
\mathcal{E}\left(g_{\mu}^{*}(x, *), g_{\mu}^{*}(y, *)\right)=g_{\mu}^{*}(x, y)=\sum_{i=1}^{N-1} \frac{\phi_{i}(x) \phi_{i}(y)}{\lambda_{i}(\mu)}, \quad x, y \in K .
$$

Now we define an operator on $L^{2}(K, \mu)$ by

$$
\mathcal{G}_{\mu} u(x)=\int_{K} g_{\mu}^{*}(x, y) u(y) d \mu(y), \quad u \in L^{2}(K, \mu) .
$$

Then if we express a function $u \in \overline{D\left[\mathcal{E}_{K}^{*}\right]}\left(\subset L^{2}(K, \mu)\right)$ by the Fourier expansion with respect to the basis $\left\{\phi_{i}\right\}$ as

$$
u=\sum_{i=0}^{N-1} \int_{K} u \phi_{i} d \mu \phi_{i} \quad\left(\text { in } L^{2}(K, \mu)\right)
$$

then we have

$$
\mathcal{G}_{\mu} u=\sum_{i=1}^{N-1} \frac{1}{\lambda_{i}(\mu)} \int_{K} u \phi_{i} d \mu \phi_{i}
$$

and also

$$
\mathcal{L}_{\mu} u=\sum_{i=1}^{N-1} \lambda_{i}(\mu) \int_{K} u \phi_{i} d \mu \phi_{i}
$$

if $u \in D\left[\mathcal{L}_{\mu}\right]$. Thus we have the following

Proposition 3.2 Under condition (3.1), $\mathcal{G}_{\mu}$ is the Green operator of $\mathcal{L}_{\mu}$, that is, it satisfies

$$
\begin{aligned}
I & =\mathcal{H}_{\mu}+\mathcal{L}_{\mu} \mathcal{G}_{\mu} \quad \text { on } \overline{D\left[\mathcal{E}_{K}^{*}\right]}\left(\subset L^{2}(K, \mu)\right), \\
I & =\mathcal{H}_{\mu}+\mathcal{G}_{\mu} \mathcal{L}_{\mu} \quad \text { on } D\left[\mathcal{L}_{\mu}\right]\left(\subset D\left[\mathcal{E}_{K}^{*}\right]\right), \\
\mathcal{H}_{\mu} \mathcal{G}_{\mu} & =\mathcal{G}_{\mu} \mathcal{H}_{\mu}=0,
\end{aligned}
$$

where

$$
\mathcal{H}_{\mu} u=\frac{1}{\mu(K)} \int_{K} u d \mu
$$


Now we shall prove the following

Proposition 3.3 Let $\mu$ be a Borel measure supported on a subset $K$ of $X$. Suppose that $K$ is finite, and regard the measure $\mu$ as a positive function on $K$. Then one has

$$
R_{\mathcal{E}}(x, y) \leq \frac{2 \max \left\{\mu(x)^{-1}, \mu(y)^{-1}\right\}}{\lambda_{1}(\mu)} \quad \text { for all } x, y \in K .
$$

Proof. For a function $u \in D\left[\mathcal{E}_{K}^{*}\right]$ and points $x, y \in K$, we have

$$
\begin{aligned}
\mid u(x) & -\left.u(y)\right|^{2} \leq 2\left(\left|u(x)-\mathcal{H}_{\mu} u\right|^{2}+\left|u(y)-\mathcal{H}_{\mu} u\right|^{2}\right) \\
& \leq 2 \max \left\{\mu(x)^{-1}, \mu(y)^{-1}\right\}\left(\left|u(x)-\mathcal{H}_{\mu} u\right|^{2} \mu(x)+\left|u(y)-\mathcal{H}_{\mu} u\right|^{2} \mu(y)\right) \\
& \leq 2 \max \left\{\mu(x)^{-1}, \mu(y)^{-1}\right\} \int_{K}\left|u-\mathcal{H}_{\mu} u\right|^{2} d \mu \\
& \leq \frac{2 \max \left\{\mu(x)^{-1}, \mu(y)^{-1}\right\}}{\lambda_{1}(\mu)} \mathcal{E}_{K}^{*}(u) .
\end{aligned}
$$

This proves the inequality of the proposition.

The monotonicity of $\lambda_{1}\left(\mathcal{E}_{K}^{*} ; \mu\right)$ with respect to finitely supported measures $\mu$ is described in the following

Lemma 3.4 Let $\mu$ and $\nu$ be respectively measures supported on finite subsets $K$ and $L$ of $X$. Suppose that $K \subset L$ and $\mu \leq \nu$ on $K$. Then one has

$$
\frac{1}{\lambda_{1}\left(\mathcal{E}_{K}^{*} ; \mu\right)} \leq \frac{1}{\lambda_{1}\left(\mathcal{E}_{L}^{*} ; \nu\right)} .
$$

Proof. Given $u \in D\left[\mathcal{E}_{K}^{*}\right]$, we denote by $v$ the restriction of the minimizer $H_{K ; u}$ to $L$. Then we have

$$
\begin{aligned}
\lambda_{1}\left(\mathcal{E}_{L}^{*} ; \nu\right) & \int_{K}\left|u-\mathcal{H}_{\mu} u\right|^{2} d \mu \leq \lambda_{1}\left(\mathcal{E}_{L}^{*} ; \nu\right) \int_{K}\left|u-\mathcal{H}_{\nu} v\right|^{2} d \mu \\
& \leq \lambda_{1}\left(\mathcal{E}_{L}^{*} ; \nu\right) \int_{K}\left|u-\mathcal{H}_{\nu} v\right|^{2} d \nu \leq \lambda_{1}\left(\mathcal{E}_{L}^{*} ; \nu\right) \int_{L}\left|v-\mathcal{H}_{\nu} v\right|^{2} d \nu \\
& \leq \mathcal{E}_{L}^{*}(v)=\mathcal{E}\left(H_{K ; u}\right)=\mathcal{E}_{K}^{*}(u) .
\end{aligned}
$$

This holds for all $u \in D\left[\mathcal{E}_{K}^{*}\right]$, and hence we get $\lambda_{1}\left(\mathcal{E}_{K}^{*} ; \mu\right) \geq \lambda_{1}\left(\mathcal{E}_{L}^{*} ; \nu\right)$.

Definition 3.1 Let $\mu_{X}^{c}$ be the counting measure on $X$ and

$$
\lambda_{1}^{*}\left(\mathcal{E} ; \mu_{X}^{c}\right)=\inf _{K} \lambda_{1}\left(\mathcal{E}_{K}^{*} ; \mu_{K}^{c}\right),
$$

where $K$ ranges over all finite subsets of $X$. 
Theorem 3.5 Consider a quadratic form $\mathcal{E}$ on a set $X$ satisfying [H-1], $[\mathrm{H}-2]$, [H-4] and [H-6] and suppose that $\lambda_{1}^{*}\left(\mathcal{E} ; \mu_{X}^{c}\right)$ is positive. Then the following assertions hold.

(i) For all $x, y \in X$,

$$
R_{\mathcal{E}}(x, y) \leq \frac{2}{\lambda_{1}^{*}\left(\mathcal{E} ; \mu_{X}^{c}\right)}
$$

(ii) The set of accumulation points $X^{d}$ of the metric space $(X, d)$ is empty, or consists of a single point $p_{\infty}$.

(iii) For any $u \in D[\mathcal{E}]$, there exists a constant $a(u)$ such that $u\left(x_{n}\right)$ tends to $a(u)$ as $n \rightarrow \infty$ for any sequence $\left\{x_{n}\right\}$ in $X$ which diverges or converges to $p_{\infty}$ if $X^{d}$ is not empty (in this case, $a(u)=u\left(p_{\infty}\right)$ ), and moreover one has

$$
\lambda_{1}^{*}\left(\mathcal{E} ; \mu_{X}^{c}\right) \int_{X}|u-a(u)|^{2} d \mu_{X}^{c} \leq \mathcal{E}(u)
$$

Proof. The assertion (i) is an immediate consequence from Proposition 3.3 and Lemma 3.4.

Let us now prove the remaining assertions. Observe first that any $u \in$ $D[\mathcal{E}]$ is bounded on $X$, since $R_{\mathcal{E}}$ is bounded (see Remark 1.1 (iii)). Let us take an increasing sequence of finite subsets $W_{n}$ of $X$ in such a way that $W=\cup W_{n}$ is dense in $X$. We write $\mu_{n}$ for $\mu_{W_{n}}^{c}$, and given $u \in D[\mathcal{E}]$, we put $u_{n}=u_{\mid W_{n}}$ and further $a_{n}=\mathcal{H}_{\mu_{n}} u_{n}\left(=\sum_{x \in W_{n}} u(x) / \sharp W_{n}\right)$. Then we have

$$
\begin{aligned}
\mathcal{E}(u) & \geq \mathcal{E}_{W_{n}}^{*}\left(u_{n}\right) \\
& \geq \lambda_{1}\left(\mathcal{E}_{W_{n}}^{*} ; \mu_{n}^{c}\right) \sum_{x \in W_{n}}\left|u_{n}(x)-a_{n}\right|^{2} \\
& \geq \lambda_{1}^{*}\left(\mathcal{E} ; \mu_{X}^{c}\right) \sum_{x \in W_{n}}\left|u_{n}(x)-a_{n}\right|^{2} .
\end{aligned}
$$

Since $u$ is bounded, $a_{n}$ is also bounded as $n \rightarrow \infty$, so that we may assume that $a_{n}$ tends to a number $a$ as $n \rightarrow \infty$. Then for any finite subset $K$ of $W$, we get

$$
\mathcal{E}(u) \geq \lambda_{1}^{*}\left(\mathcal{E} ; \mu_{X}^{c}\right) \sum_{x \in K}|u(x)-a|^{2} .
$$

This holds for $W$. Moreover if we have an accumulation point $p \in X^{d}$, then $u\left(x_{k}\right)$ tends to $a$ for all $x_{k}$ in $W$ converging to $p$, and hence $a=u(p)$. This verifies that if we have $p, q \in X^{d}$, then $u(p)=u(q)$ for all $u \in D[\mathcal{E}]$, which implies that $R_{\mathcal{E}}(p, q)=0$ and hence $p=q$. this completes the proof of Theorem 3.5. 
In what follows, we assume that $\mathcal{E}$ is a resistance form. Let $K$ be a finite subset of $X$. Then the characteristic functions $\chi_{x}$ for all $x \in K$ belong to $D\left[\mathcal{E}_{K}^{*}\right]$. Set $c(x, y)=-\mathcal{E}_{K}^{*}\left(\chi_{x}, \chi_{y}\right)$ for $x, y \in K, x \neq y$, and $\pi_{K}(x)=\mathcal{E}_{K}^{*}\left(\chi_{x}, \chi_{x}\right)\left(=\sum_{y \in K, y \neq x} c(x, y)\right)$ for $x \in K$. Define a measure $\mu_{K}^{p}$ on $K$ by

$$
\int u d \mu_{K}^{p}=\sum_{x \in K} u(x) \pi_{K}(x), \quad u \in \ell(K) .
$$

Then the operator on $L^{2}\left(K, \mu_{K}^{p}\right)$, denoted by $\mathcal{L}_{\mu}^{p}$, is expressed as follows:

$$
\mathcal{L}_{K}^{p} u(x)=\frac{1}{\pi_{K}(x)} \mathcal{L}^{c} u(x)=u(x)-\mathcal{M}_{K} u(x),
$$

where we put

$$
\mathcal{M}_{K} u(x)=\frac{1}{\pi_{K}(x)} \sum_{y \in K, y \neq x} c(x, y) u(y) .
$$

We remark here that

$$
\min _{x \in K} \pi_{K}(x) \lambda_{1}\left(\mu_{K}^{p}\right) \leq \lambda_{1}\left(\mu_{K}^{c}\right) \leq \max _{x \in K} \pi_{K}(x) \lambda_{1}\left(\mu_{K}^{p}\right) .
$$

By the same arguments as in Lemma 3.4, we have the following

Lemma 3.6 Let $K$ and $L$ be finite subsets of $X$ such that $K \subset L$. Then $\pi_{K} \leq \pi_{L}$ on $K$ and $\lambda_{1}\left(\mathcal{E}_{K}^{*} ; \mu_{K}^{p}\right) \geq \lambda_{1}\left(\mathcal{E}_{L}^{*} ; \mu_{L}^{p}\right)$.

Definition 3.2 Let

$$
\pi_{X}(x):=\sup _{K} \pi_{K}(x), \quad x \in X ; \quad \lambda_{1}^{*}\left(\mathcal{E} ; \mu_{X}^{p}\right):=\inf _{K} \lambda_{1}\left(\mathcal{E}_{K}^{*} ; \mu_{K}^{p}\right),
$$

where $K$ ranges over all finite subsets of $X$.

Theorem 3.7 Let $\mathcal{E}$ be a resistance form on a set $X$ satisfying [H-6] and suppose that $\pi_{X}(x)$ is finite for all $x \in X$ and $\lambda_{1}^{*}\left(\mathcal{E} ; \mu_{X}^{p}\right)$ is positive. Then the following assertions hold.

(i) For all $x, y \in X$,

$$
R_{\mathcal{E}}(x, y) \leq \frac{2 \max \left\{\pi_{X}(x)^{-1}, \pi_{X}(y)^{-1}\right\}}{\lambda_{1}^{*}\left(\mathcal{E} ; \mu_{X}^{p}\right)} .
$$

(ii) The set of accumulation points $X^{d}$ of the metric space $(X, d)$ is empty, or consists of a single point $p_{\infty}$.

(iii) For any $u \in D[\mathcal{E}]$, there exists a constant $a(u)$ such that $u\left(x_{n}\right)$ tends to $a(u)$ as $n \rightarrow \infty$ for any sequence $\left\{x_{n}\right\}$ in $X$ which diverges or converges 
to $p_{\infty}$ if $X^{d}$ is not empty (in this case, $a(u)=u\left(p_{\infty}\right)$ ), and moreover one has

$$
\lambda_{1}^{*}\left(\mathcal{E} ; \mu_{X}^{p}\right) \int_{X}|u-a(u)|^{2} d \mu_{X}^{p} \leq \mathcal{E}(u) .
$$

Remark 3.1 (i) Let $\mathcal{E}$ be a resistance form on a finite or countably infinite set $X$ such that $\pi_{X}(x)$ is finite for any $x \in X$. A point of $X$ is called a vertex, and a vertex $x$ is, by definition, adjacent to another $y$ if $c(x, y):=$ $-\mathcal{E}\left(\chi_{x}, \chi_{y}\right)>0$. If this is the case, $x$ and $y$ are assumed to be joined by an edge of resistance $r(|x y|)=c(x, y)^{-1}$. In this way, we get a connected network $(X, E, r)$, where $E$ stands for the set of edges, each of which is assigned the positive number $r$.

(ii) Let $\mathcal{E}$ be a quadratic from on a finite or countably infinite set $X$ satisfying [H-2] and [H-5]. The spectral gap of $\mathcal{E}$ in $L^{2}\left(X, \mu^{c}\right)$ is given by the number $\lambda_{0}\left(\mathcal{E} ; \mu^{c}\right)=\inf \left\{\mathcal{E}(u) \mid u \in D[\mathcal{E}], \sum_{x \in X} u(x)^{2}=1\right\}$. When $\lambda_{0}\left(\mathcal{E} ; \mu^{c}\right)$ is positive, we have $\sup _{x \in X} g_{\mathcal{E}}(x, x) \leq 2 / \lambda_{0}\left(\mathcal{E} ; \mu^{c}\right)$.

3.2 In this part we investigate a resistance form $\mathcal{E}$ on a set $X$ satisfying condition $[\mathrm{H}-6]$.

Suppose $\mu$ is a Borel measure supported on a closed subset $K$ of $X$ such that

$$
\mu(K)<\infty ; \iint_{K \times K} R_{\mathcal{E}}(x, y)^{2} d \mu(x) d \mu(y)<+\infty .
$$

Then we introduce a nonnegative symmetric function on $K \times K$ by

$$
\mathcal{N}_{\mu}(x, y):=\left(\frac{1}{\mu(K)} \int_{K}\left(g_{\mu}(x, z)-g_{\mu}(y, z)\right)^{2} d \mu(z)\right)^{1 / 2}, \quad x, y \in K .
$$

It is evident that $\mathcal{N}_{\mu}$ satisfies the triangle inequality, and in fact it defines a distance on $K$ (see (3.8) below).

Let $\Phi=\left\{\phi_{i} \mid i=0,1, \ldots, N-1\right\}$ be a complete orthonormal system of eigenfunctions $\phi_{i}$ of $\mathcal{L}_{\mu}$ with the $i$-th eigenvalues $\lambda_{i}(\mu)$, where $N, 1 \leq$ $N \leq+\infty$, stands for the cardinality of $K$. In what follows, we understand $\phi_{i} / \sqrt{\lambda_{i}(\mu)}=0$ for $i \geq N$ if $N$ is finite, and for simplicity, we assume $N=\infty$. Recall that

$$
R_{\mathcal{E}}(x, y)=\sum_{i=1}^{\infty}\left(\frac{\phi_{i}(x)}{\sqrt{\lambda_{i}(\mu)}}-\frac{\phi_{i}(y)}{\sqrt{\lambda_{i}(\mu)}}\right)^{2}, \quad x, y \in K .
$$

Relevantly to this expression, we define a map $I_{\Phi}$ of $K$ into the Hilbert space of square summable sequences, $\ell^{2}=\left\{\left(a_{i}\right) \mid \sum_{i=1}^{\infty} a_{i}^{2}<+\infty\right\}$, by

$$
I_{\Phi}(x)=\left(\frac{\phi_{i}(x)}{\sqrt{\lambda_{i}(\mu)}}\right), \quad x \in K
$$


Then $I_{\Phi}$ gives rise to an isometric embedding of the metric subspace $\left(K, \sqrt{R_{\mathcal{E}}}\right)$ of $\left(X, \sqrt{R_{\mathcal{E}}}\right)$ into $\ell^{2}$; in fact, it satisfies

$$
R_{\mathcal{E}}(x, y)=\left\|I_{\Phi}(x)-I_{\Phi}(y)\right\|_{\ell^{2}}^{2} ; g_{\mu}^{*}(x, y)=\left(I_{\Phi}(x), I_{\Phi}(y)\right)_{\ell^{2}}, \quad x, y \in K
$$

Then the distance $\mathcal{N}_{\mu}$ can be expressed by

$$
\begin{aligned}
\mathcal{N}_{\mu}(x, y) & =\left(\frac{1}{\mu(K)} \int_{K}\left(\sum_{i=1}^{\infty} \frac{1}{\lambda_{i}(\mu)}\left(\phi_{i}(x)-\phi_{i}(y)\right) \phi_{i}(z)\right)^{2} d \mu(z)\right)^{1 / 2} \\
& =\left(\frac{1}{\mu(K)} \int_{K}\left(I_{\Phi}(x)-I_{\Phi}(y), I_{\Phi}(z)\right)_{\ell^{2}}^{2} d \mu(z)\right)^{1 / 2}
\end{aligned}
$$

Then by the Cauchy-Schwarz inequality, we get

$$
\begin{aligned}
\mathcal{N}_{\mu}(x, y) & \leq\left(\frac{1}{\mu(K)} \int_{K} g_{\mu}^{*}(z, z) d \mu(z)\right)^{1 / 2} R_{\mathcal{E}}(x, y)^{1 / 2} \\
& =\left(\frac{1}{\mu(K)} \sum_{i=1}^{N-1} \frac{1}{\lambda_{i}(\mu)}\right)^{1 / 2} R_{\mathcal{E}}(x, y)^{1 / 2}
\end{aligned}
$$

We observe here that a function $u \in D\left[\mathcal{L}_{\mu}\right]$ is Lipschitz continuous with respect to $\mathcal{N}_{\mu}$. In fact, we have by Proposition 3.2

$$
\begin{aligned}
& |u(x)-u(y)|=\left|\int_{K}\left(g_{\mu}^{*}(x, z)-g_{\mu}^{*}(y, z)\right) \mathcal{L}_{\mu} u(z) d \mu(z)\right| \\
& \quad \leq\left(\frac{1}{\mu(K)} \int_{K}\left(g_{\mu}(x, z)-g_{\mu}(y, z)\right)^{2} d \mu(z)\right)^{1 / 2}\left(\mu(K) \int_{K}\left|\mathcal{L}_{\mu} u\right|^{2} d \mu(z)\right)^{1 / 2},
\end{aligned}
$$

and hence

$$
|u(x)-u(y)| \leq\left(\mu(K) \int_{K}\left|\mathcal{L}_{\mu} u\right|^{2} d \mu\right)^{1 / 2} \mathcal{N}_{\mu}(x, y)
$$

for all $x, y \in K$. In particular, if $\phi$ is an eigenfunction of $\mathcal{L}_{\mu}$ with eigenvalue $\lambda \neq 0$, then

$$
|\phi(x)-\phi(y)| \leq \lambda\left(\mu(K) \int_{K} \phi^{2} d \mu\right)^{1 / 2} \mathcal{N}_{\mu}(x, y) .
$$

Let us now introduce another distance $\mathcal{S}^{\mu}$ on $K$ by

$$
\mathcal{S}_{\mu}(x, y)=\left(p_{\mu}(1, x, x)-2 p_{\mu}(1, x, y)+p_{\mu}(1, y, y)\right)^{1 / 2}, \quad x, y \in K,
$$


where $p_{\mu}(t, x, y)$ denotes the kernel function of the semigroup $\exp \left(-t \mathcal{L}_{\mu}\right)$ generated by $\mathcal{L}_{\mu}$. The distance $\mathcal{S}_{\mu}$ is written as

$$
\mathcal{S}_{\mu}(x, y)=\left(\sum_{i=1}^{N-1} e^{-\lambda_{i}(\mu)}\left(\phi_{i}(x)-\phi_{i}(y)\right)^{2}\right)^{1 / 2}, \quad x, y \in K .
$$

Therefore it follows from (3.9) that

$$
\begin{aligned}
\mathcal{S}_{\mu}(x, y) & \leq\left(\mu(K) \sum_{i=1}^{\infty} \lambda_{i}(\mu)^{2} e^{-\lambda_{i}(\mu)}\right)^{1 / 2} \mathcal{N}_{\mu}(x, y) \\
& \leq C_{0}\left(\mu(K) \sum_{i=1}^{\infty} \frac{1}{\lambda_{i}(\mu)}\right)^{1 / 2} \mathcal{N}_{\mu}(x, y),
\end{aligned}
$$

where $C_{0}$ is some absolute constant. Obviously an eigenfunction $\phi$ of $\mathcal{L}_{\mu}$ with eigenvalue $\lambda$ is Lipschitz continuous with respect to $\mathcal{S}_{\mu}$; in fact, we have

$$
|\phi(x)-\phi(y)| \leq e^{\lambda / 2}\left(\int_{K} \phi^{2} d \mu\right)^{1 / 2} \mathcal{S}_{\mu}(x, y) .
$$

Relevantly to the distance $\mathcal{S}^{\mu}$, we define a map $J_{\Phi}: K \rightarrow \ell^{2}$ by

$$
J_{\Phi}(x)=\left(e^{-\lambda_{i}(\mu) / 2} \phi_{i}(x)\right), \quad x \in K,
$$

that is an isometric embedding of the metric space $\left(K, \mathcal{S}^{\mu}\right)$ into $\ell^{2}$. We denote by $\bar{K}^{\mu}$ the completion of $\left(K, \mathcal{S}^{\mu}\right)$. We notice that

$$
0 \leq g_{\mu}(x, y) \leq g_{\mu}(x, x)=\frac{1}{\mu(K)} \int_{K} R_{\mathcal{E}}(x, z) d \mu(z)
$$

and

$$
\begin{aligned}
\int_{K} g_{\mu}(z, z)^{2} d \mu(z) & =\frac{1}{\mu(K)^{2}} \int_{K}\left(\int_{K} R_{\mathcal{E}}(z, w) d \mu(w)\right)^{2} d \mu(z) \\
& \leq \frac{1}{\mu(K)} \iint_{K \times K} R_{\mathcal{E}}(z, w)^{2} d \mu(z) d \mu(w) .
\end{aligned}
$$

Using these estimates, we get

$$
\begin{aligned}
\mathcal{N}_{\mu}(x, y)^{2} & \leq \frac{2}{\mu(K)} \int_{K} g_{\mu}(z, z)^{2} d \mu(z) \\
& \leq \frac{2}{\mu(K)^{2}} \iint_{K \times K} R_{\mathcal{E}}(z, w)^{2} d \mu(z) d \mu(w)
\end{aligned}
$$

for all $x, y \in K$. 
In this way, the distance $\mathcal{N}_{\mu}$ turns out to be bounded, and hence any function of $D\left[\mathcal{L}_{\mu}\right]$ is bounded. In particular, an eigenfunction $\phi$ with nontrivial eigenvalue $\lambda$ is bounded, and in view of (3.9), it satisfies

$$
|\phi(x)|^{2} \leq \frac{2 \lambda^{2}}{\mu(K)} \iint_{K \times K} R_{\mathcal{E}}(z, w)^{2} d \mu(z) d \mu(w) \int_{K} \phi^{2} d \mu, x \in K .
$$

Lemma 3.8 The completion $\bar{K}^{\mu}$ of $\left(K, \mathcal{S}_{\mu}\right)$ is compact and any function of $D\left[\mathcal{L}_{\mu}\right]$ extends continuously to $\bar{K}^{\mu}$.

Proof. We take a divergent sequence $\left\{s_{i}\right\}$ of positive numbers, which will be appropriately chosen later, and for $r>0$, we denote by $B^{1,2}(r)(r>0)$ the subset of $\ell^{2}$ consisting of elements $\left(a_{i}\right)$ with $\sum_{i=1}^{\infty}\left(1+s_{i}\right) a_{i}^{2} \leq r^{2}$, that is a compact subset of $\ell^{2}$. We take a positive number $M$ so that

$$
\frac{1}{2}\left(\iint_{K \times K} R_{\mathcal{E}}(z, w)^{2} d \mu(z) d \mu(w)\right)^{1 / 2} \leq M .
$$

Since $\sum_{i=1}^{\infty} 1 / \lambda_{i}(\mu) \leq \frac{1}{2}\left(\iint_{K \times K} R_{\mathcal{E}}(z, w)^{2} d \mu(z) d \mu(w)\right)^{1 / 2}$ by $(3.5)$, we see that $\lambda_{i}(\mu) \geq i / M$ for all $i=1,2, \ldots$. Therefore if we set $s_{i}=i / M$, then for any $x \in K$, we have

$$
\begin{aligned}
\sum_{i=1}^{\infty}\left(1+s_{i}\right) e^{-\lambda_{i}(\mu)} \phi_{i}(x)^{2} & \leq 4 M^{2} \mu(K)^{-1} \sum_{i=1}^{\infty}\left(1+s_{i}\right) \lambda_{i}(\mu)^{2} e^{-\lambda_{i}(\mu)} \\
& \leq C_{0} M^{2} \mu(K)^{-1} \sum_{i=1}^{\infty}\left(1+s_{i}\right) e^{-\lambda_{i}(\mu) / 2} \\
& \leq C_{0} M^{2} \mu(K)^{-1} \sum_{i=1}^{\infty}\left(1+s_{i}\right) e^{-s_{i} / 2}
\end{aligned}
$$

where $C_{0}$ is some absolute constant. Letting $r(M)^{2}=C_{0} M^{2} \mu(K)^{-1} \sum_{i=1}^{\infty}(1+$ $\left.s_{i}\right) e^{-s_{i}}$, we conclude that $J_{\Phi}(K)$ is included in $B^{1,2}(r(M))$ : in particular, $\left(\bar{K}^{\mu}, \mathcal{S}^{\mu}\right)$ turns out to be compact. Moreover given $u \in D\left[\mathcal{L}_{\mu}\right]$, we put $u_{n}=\sum_{i=0}^{n} \int_{K} u \phi_{i} d \mu \phi_{i}$. Taking a point $y \in K$ in such a way that $u(y)-u_{n}(y)=0$, applying (3.8) and using (3.11), we obtain

$$
\begin{gathered}
\sup _{x \in K}\left|u(x)-u_{n}(x)\right|^{2} \leq \mu(K)\left(\int_{K}\left(\sum_{i=n+1}^{\infty} \lambda_{i}(\mu) \int_{K} u \phi_{i} d \mu \phi_{i}\right)^{2} d \mu\right) \sup _{x \in K} \mathcal{N}_{\mu}(x, y)^{2} \\
=\frac{2}{\mu(K)} \iint_{K \times K} R_{\mathcal{E}}(z, w)^{2} d \mu(z) d \mu(w)\left(\sum_{i=n+1}^{\infty} \lambda_{i}(\mu)^{2}\left(\int_{K} u \phi_{i} d \mu\right)^{2}\right) .
\end{gathered}
$$

Since the right side tends to zero as $n$ goes to infinity, we see that every $u \in$ $D\left[\mathcal{L}_{\mu}\right]$ is continuous relative to the distance $\mathcal{S}_{\mu}$ and extends to a continuous function on $\bar{K}^{\mu}$. 
The measure $\mu$ can be taken to be a Radon measure on the compact metric space $\bar{K}^{\mu}$, since the inclusion map from $\left(K, d_{\mid K}\right)$ to $\left(\bar{K}^{\mu}, \mathcal{S}_{\mu}\right)$ is continuous, and the form $\left(\mathcal{E}_{K}^{*}, D\left[\mathcal{E}_{K}^{*}\right]\right)$ also can be considered as a Dirichlet form on $L^{2}\left(\bar{K}^{\mu}, \mu\right)$. Moreover $D\left[\mathcal{E}_{K}^{*}\right] \cap C\left(\bar{K}^{\mu}\right)$ is an algebra containing the unit element 1 and $D\left[\mathcal{L}_{\mu}\right]$ that separates points of $\bar{K}^{\mu}$, and hence $D\left[\mathcal{E}_{K}^{*}\right] \cap C\left(\bar{K}^{\mu}\right)$ is dense in $C\left(\bar{K}^{\mu}\right)$ with uniform norm, and it is clearly dense in $D\left[\mathcal{E}_{K}^{*}\right]$ with norm $\mathcal{E}_{K}^{* 1 / 2}+\|\cdot\|_{L^{2}}$. Thus we have the following

Theorem 3.9 The Dirichlet form $\left(\mathcal{E}_{K}^{*}, D\left[\mathcal{E}_{K}^{*}\right]\right)$ on $L^{2}\left(\bar{K}^{\mu}, \mu\right)$ is regular.

We refer the reader to e.g. [21] for the theory on Dirichlet spaces.

Let

$Q\left(K, \mathcal{E}_{K}^{*}\right)=$ the space of functions on $K$ spanned by $\left\{g_{z}(x, *) \mid x, z \in K\right\}$.

We observe that $u \in D\left[\mathcal{E}_{K}^{*}\right]$ belongs to $Q\left(K, \mathcal{E}_{K}^{*}\right)$ if and only if there exists a finite subset $A$ of $K$ such that $\mathcal{E}_{K}^{*}(u, v)=0$ for every $v \in D\left[\mathcal{E}_{K}^{*}\right]$ that vanishes on $A$. We note also that $Q\left(K, \mathcal{E}_{K}^{*}\right)$ is included in the family of functions spanned by $\left\{g_{\mu}(x, *) \mid x \in K\right\}$, because we have

$$
\begin{aligned}
g_{z}(x, y) & =\frac{1}{\mu(K)} \int_{K} g_{z}(x, w) d \mu(w)+\mathcal{E}\left(g_{\mu}(y, *), g_{z}(x, *)\right) \\
& =\frac{1}{\mu(K)} \int_{K} g_{z}(x, w) d \mu(w)+g_{\mu}(y, x)-g_{\mu}(y, z) .
\end{aligned}
$$

Now we suppose that for each $x \in K$, there exists a positive constant $c_{x}$ such that

$$
|u(x)| \leq c_{x}\left(\int_{K} u^{2} d \mu\right)^{1 / 2}, \quad \forall u \in D\left[\mathcal{E}_{K}^{*}\right] .
$$

Since $u(x)=\mathcal{H}_{\mu} u+\mathcal{E}\left(u, g_{\mu}(x, *)\right)$, this is equivalent to the condition that $g_{\mu}(x, *) \in D\left[\mathcal{L}_{\mu}\right]$ for all $x \in K$, that is, $\sum_{i=1}^{\infty} \phi_{i}(x)$ is finite for all $x \in K$. In particular, for any fixed $x \in K$, the function $g_{\mu}(x, y)$ of $y \in K$ continuously extends to $\bar{K}^{\mu}$ and $g_{\mu}(x, y)$ is bounded from above by $g_{\mu}(y, y)$ that is square summable, since we have

$$
\begin{aligned}
g_{\mu}(y, y) & =\int_{K} g_{z}(y, y) d \mu(z)=\int_{K} R_{\mathcal{E}}(z, y) d \mu(z) \\
& \leq \int_{K} R_{\mathcal{E}}(z, o) d \mu(z)+R_{\mathcal{E}}(0, y) \mu(K),
\end{aligned}
$$

where $o$ is a fixed point of $K$. This, together with Lebesgue's convergence theorem, shows that the distance $\mathcal{N}_{\mu}$ also extends continuously to $\bar{K}^{\mu}$. In this way, under the assumption (3.12), we have verified that the completion $\bar{K}^{N}$ of $K$ with respect to the distance $\mathcal{N}_{\mu}$ topologically coincides with $\bar{K}^{\mu}$. 
Moreover $Q\left(K, \mathcal{E}_{K}^{*}\right)$ is taken to belong to $C\left(\bar{K}^{\mu}\right)$ and $Q\left(K, \mathcal{E}_{K}^{*}\right)$ separates points of $\bar{K}^{N}$, so that the algebra generated by $Q\left(K, \mathcal{E}_{K}^{*}\right)$ is dense in $C\left(\bar{K}^{\mu}\right)$. Thus we have the following

Theorem 3.10 Under the assumption (3.12), the completion $\bar{K}^{N}$ is compact and determined topologically by $Q\left(K, \mathcal{E}_{K}^{*}\right)$; moreover $\left(\mathcal{E}_{K}^{*}, D\left[\mathcal{E}_{K}^{*}\right]\right)$ is a regular Dirichlet form on $L^{2}\left(\bar{K}^{N}, \mu\right)$.

Now we suppose, instead of (3.12), that the effective resistance $R_{\mathcal{E}}$ of $\mathcal{E}$ is bounded on $K$, that is $R_{\mathcal{E}}(x, y) \leq D$ for some positive constant $D$ and all $x, y \in K$. In this case, we have

$$
|u(x)-u(y)|^{2} \leq D \mathcal{E}_{K}^{*}(u)
$$

for all $u \in D\left[\mathcal{E}_{K}^{*}\right]$ and $x, y \in K$. Therefore given $u \in D\left[\mathcal{E}_{K}^{*}\right]$ and $u_{n}=$ $\sum_{i=0}^{n} \int_{K} u \phi_{i} d \mu \phi_{i}$, we take a point $y \in K$ in such a way that $u(y)-u_{n}(y)=0$ and get

$$
\sup _{x \in K}\left|u(x)-u_{n}(x)\right|^{2} \leq D\left(\sum_{i=n+1}^{\infty} \lambda_{i}(\mu)\left(\int_{K} u \phi_{i} d \mu\right)^{2}\right) .
$$

The right side tends to zero as $n \rightarrow \infty$. Thus $u_{n}$ uniformly converges to $u$ on $K$ and any $u_{n}$ extend to a continuous function on $\bar{K}^{\mu}$, and so does $u$ itself. Thus $D\left[\mathcal{E}_{K}^{*}\right]$ may be regarded as a subspace of the Banach space $C\left(\bar{K}^{\mu}\right)$ and the distance $\mathcal{N}_{\mu}$ also yields the same topology on $\bar{K}^{\mu}$ as $\mathcal{S}_{\mu}$. Moreover for any $x, y \in \bar{K}^{N},|u(x)-u(y)|^{2} \leq D^{2} \mathcal{E}_{K}^{*}(u)$ for all $u \in D\left[\mathcal{E}_{K}^{*}\right]$. This implies that $\overline{\mathcal{E}}_{K}^{*}$ satisfies $[\mathrm{H}-1]$ :

$$
0<R_{\mathcal{E}_{K}^{*}}(x, y)=\sup \left\{\frac{|\hat{u}(x)-\hat{u}(y)|^{2}}{\overline{\mathcal{E}}_{K}^{*}(\hat{u})} \mid \overline{\mathcal{E}}_{K}^{*}(\hat{u})>0\right\} \leq D^{2}<+\infty
$$

for all $x, y \in \bar{K}^{N}, x \neq y$, and moreover it inherits the properties [H-2] through $[\mathrm{H}-7]$ except $[\mathrm{H}-5]$. We note here that $\mathcal{N}_{\mu}(\bar{x}, \bar{y}) \leq C R_{\mathcal{E}_{K}^{*}}(\bar{x}, \bar{y})^{1 / 2}$ for all $\bar{x}, \bar{y} \in \bar{K}^{N}$ and some constant $C$, so that the identity map $i$ : $\left(\bar{K}^{N}, R_{\mathcal{E}_{K}^{*}}\right) \rightarrow\left(\bar{K}^{N}, \mathcal{N}_{\mu}\right)$ is continuous; however it is not homeomorphic in general (see Examples 5.3).

Thus we have shown the following

Theorem 3.11 Let $(X, \mathcal{E})$ be a resistance form $\mathcal{E}$ on a set $X$ satisfying $[\mathrm{H}-6]$ and $K$ a closed subset of $X$. Suppose that the effective resistance of $\mathcal{E}_{K}^{*}$ is bounded. For a finite Borel measure $\mu$ supported on $K,\left(\bar{K}^{N}, \mathcal{N}_{\mu}\right)$ is the compact metric space to which every function of $D\left[\mathcal{E}_{K}^{*}\right]$ extends continuously and whose points are separated by $D\left[\mathcal{E}_{K}^{*}\right]$. Moreover $\left(\mathcal{E}_{K}^{*}, D\left[\mathcal{E}_{K}^{*}\right]\right)$ is a resistance form on $\bar{K}^{N}$. 
Remark 3.1 (i) The distance introduced in 3.2 can be also written as follows: for $x, y \in K$,

$$
\mathcal{N}_{\mu}(x, y)^{2}=\frac{1}{\mu(K)} \int_{K}\left(g_{y}(x, z)-\frac{1}{\mu(K)} \int_{K} g_{y}(x, w) d \mu(w)\right)^{2} d \mu(z),
$$

or

$$
\begin{aligned}
\mathcal{N}_{\mu}(x, y)^{2}=\frac{1}{\mu(K)} \int_{K} & \left(R_{\mathcal{E}}(x, z)-R_{\mathcal{E}}(y, z)\right. \\
& \left.-\frac{1}{\mu(K)} \int_{K} R_{\mathcal{E}}(x, w)-R_{\mathcal{E}}(y, w) d \mu(w)\right)^{2} d \mu(z) .
\end{aligned}
$$

(ii) The distance $\mathcal{N}_{\mu}$ on $K$ extends to $X$ and gives a pseudo distance on $X$. In addition, using the minimizers $H_{K ; \phi_{i}}$ for $\phi_{i} \in D\left[\mathcal{E}_{K}^{*}\right]$ $(i=0,1,2, \ldots)$, and letting $\hat{p}^{\mu}(t, x, y)=\sum_{i=0}^{\infty} e^{-t \lambda_{i}} H_{K ; \phi_{i}}(x) H_{K ; \phi_{i}}(y)$ for $x, y \in X$, we obtain a pseudo distance $\hat{S}^{\mu}$ on $X$ defined by $\hat{S}^{\mu}(x, y)=$ $\left(\hat{p}^{\mu}(1, x, x)-2 \hat{p}^{\mu}(1, x, y)+\hat{p}^{\mu}(1, y, y)\right)^{1 / 2}$ for $x, y \in X$. Then $\hat{S}^{\mu}$ is also bounded by $\mathcal{N}_{\mu}$ on $X \times X$ as in (3.10).

(iii) In Theorem 3.10, condition (3.12) is always satisfied if the closed subset $K$ under consideration is discrete in $(X, d)$.

(iv) In Theorem 3.11, the semigroup $P_{t}=\exp \left(-t \mathcal{L}_{\mu}\right)$ induces a contraction semigroup of class $\left(C_{0}\right)$ on the Banach space $C\left(\bar{K}^{N}\right)$, i.e., $P_{t}\left(C\left(\bar{K}^{N}\right) \subset\right.$ $C\left(\bar{K}^{N}\right)$ and for $f \in C\left(\bar{K}^{N}\right), \lim _{t \rightarrow 0} P_{t} f=f$ in $C\left(\bar{K}^{N}\right)$.

\section{Infinite networks}

In this section, we introduce the Royden and the Kuramochi compactifications of infinite networks and illustrate some properties of these compactifications.

A compactification of any (discrete) set $X$ is a compact Hausdorff space which contains $X$ as a dense subset and which induces the discrete topology on $X$. It is known that given a family $\Phi$ of bounded functions on $X$, there exists an (up to canonical homeomorphism) unique compactification $\mathcal{C}(X, \Phi)$ of $X$ with the following properties (cf. e.g. [12]): (i) every function of $\Phi$ extends to a continuous function on $\mathcal{C}(X, \Phi)$ and (ii) the extended functions separate the points of the boundary $\partial \mathcal{C}(X, \Phi)=\mathcal{C}(X, \Phi) \backslash V$. We remark that if $\Psi$ is a subfamily of $\Phi$, then the identity map extends to a continuous map from $C(X, \Phi)$ onto $\mathcal{C}(X, \Psi)$, and if $\Phi_{0}$ is a subfamily of $\Phi$ and each function of $\Phi$ is a finite linear combination of functions in $\Phi_{0}$, then $C(X, \Phi)$ and $C\left(X, \Phi_{0}\right)$ are canonically homeomorphic; in particular, if in addition, $X$ and $\Phi_{0}$ is countable, then $C(X, \Phi)$ is metrizable. 
Now we consider a connected, infinite graph $G=(V, E)$. The compactification relative to the space of bounded, locally constant functions is called the end compactification of the graph. When a positive function $r$ on $E$ is given, we have a distance $d_{r}$ on $V$. The compactification with respect to the space of bounded Lipschitz functions on $\left(V, d_{r}\right)$ is denoted by $\mathcal{L}_{b}\left(V, d_{r}\right)$. We have the compactification relative to $Q\left(V, \mathcal{E}_{\Gamma}\right)$, called the Kuramochi compactification of the network $\Gamma=(V, E, r)$ in [35] and [36]. This can be topologically identified with the compact metric space $\left(\bar{V}^{N}, \mathcal{N}_{\mu}\right)$ as shown in Theorem 3.10. We denote by $\bar{V}^{N}\left(\mathcal{E}_{\Gamma}\right)$ and $\partial \bar{V}^{N}\left(\mathcal{E}_{\Gamma}\right)$ respectively the Kuramochi compactification and the Kuramochi boundary of the network $\Gamma$. Some important properties of this compactification will be discussed in 7.3.

The compactification relative to the space of bounded functions in $D\left[\mathcal{E}_{\Gamma}\right]$, $B D\left[\mathcal{E}_{\Gamma}\right]$, is called the Royden compactification of the network $\Gamma$ and denoted by $\mathcal{R}\left(V, \mathcal{E}_{\Gamma}\right)$. The boundary $\partial \mathcal{R}\left(V, \mathcal{E}_{\Gamma}\right)$ is called the Royden boundary of $\Gamma$. There is an important part of the Royden boundary referred to as the harmonic boundary of $\Gamma$ which is defined by

$$
\Delta\left(\mathcal{E}_{\Gamma}\right)=\left\{x \in \partial \mathcal{R}\left(V, \mathcal{E}_{\Gamma}\right) \mid g(x)=0 \quad \text { for all } g \in B D_{0}\left[\mathcal{E}_{\Gamma}\right]\right\} .
$$

The following duality holds for the harmonic boundary (cf. [40] and [37, Chap. VI]):

$$
B D_{0}\left[\mathcal{E}_{\Gamma}\right]=\left\{g \in B D\left[\mathcal{E}_{\Gamma}\right] \mid g(x)=0 \quad \text { for all } x \in \Delta\left(\mathcal{E}_{\Gamma}\right)\right\} .
$$

It is known (cf. [40], [31], [37, Chap. VI]) that $\Gamma$ is nonparabolic, i.e., $\left(\mathcal{E}_{\Gamma}^{0}, D_{0}\left[\mathcal{E}_{\Gamma}\right]\right)$ satisfies $[\mathrm{H}-5]$ if and only if the harmonic boundary is not empty, and also that if $\partial \mathcal{R}\left(V, \mathcal{E}_{\Gamma}\right) \backslash \Delta\left(\mathcal{E}_{\Gamma}\right)$ is nonempty, then any set of a single point there is not a $G_{\delta}$ set and for a nonempty closed subset $F$ in $\partial \mathcal{R}\left(V, \mathcal{E}_{\Gamma}\right) \backslash \Delta\left(\mathcal{E}_{\Gamma}\right)$, there exists a function $g \in D_{0}\left[\mathcal{E}_{\Gamma}\right]$ such that $g(x)$ tends to infinity as $x \in V \rightarrow F$. In view of this property, we have the following

Proposition 4.1 The following conditions are mutually equivalent:

(i) $\sup _{x \in V} g_{\Gamma}^{0}(x, x)$ is finite.

(ii) $D_{0}\left[\mathcal{E}_{\Gamma}\right]=B D_{0}\left[\mathcal{E}_{\Gamma}\right]$, that is, any $g \in D_{0}\left[\mathcal{E}_{\Gamma}\right]$ is bounded.

(iii) $\partial \mathcal{R}\left(V, \mathcal{E}_{\Gamma}\right)=\Delta\left(\mathcal{E}_{\Gamma}\right)$, that is, for any $g \in B D_{0}\left[\mathcal{E}_{\Gamma}\right], g(x)$ tends to zero as $x \in V \rightarrow \infty$.

(iv) For any $g \in D_{0}\left[\mathcal{E}_{\Gamma}\right], g(x)$ tends to zero as $x \in V \rightarrow \infty$.

Proof. Obviously (i) (resp. (iv)) implies (ii) (resp. (ii)). Suppose that (ii) holds. Then the bounded inverse theorem shows that the two norms $\mathcal{E}_{\Gamma}(u)^{1 / 2}$ $+|u(o)|$ and $\mathcal{E}_{\Gamma}(u)^{1 / 2}+\|u\|_{\infty}$ for $u \in D_{0}\left[\mathcal{E}_{\Gamma}\right]$ are equivalent, so that $\|u\|_{\infty} \leq$ 
$M \mathcal{E}_{\Gamma}(u)^{1 / 2}$ for some positive constant $M$ and all $u \in D_{0}\left[\mathcal{E}_{\Gamma}\right]$. This shows that $\sup _{x \in V} g_{\Gamma}(x, x) \leq M$, and thus (ii) implies (i). It follows now from the fact mentioned above that (ii) implies (iii). Finally we get (iv) from (iii), since $g_{n}=\min \{\max \{g,-n\}, n\} \in B D_{0}\left[\mathcal{E}_{\Gamma}\right]$ for any $g \in D_{0}\left[\mathcal{E}_{\Gamma}\right]$ and a positive constant $n>0$. This completes the proof of Proposition 4.1.

We remark that the identity map of $V$ induces a surjective continuous map from $\mathcal{L}_{b}\left(V, d_{r}^{1 / 2}\right)$ onto the Royden boundary, since $B D\left[\mathcal{E}_{\Gamma}\right]$ belongs to the space of bounded Lipschitz continuous functions on $\left(V, d_{r}^{1 / 2}\right)$, and moreover that if the diameter of $\left(V, d_{r}^{1 / 2}\right)$ is bounded, then by (1.6), the effective resistance of $\Gamma$ is also bounded. This is equivalent to the condition that $D\left[\mathcal{E}_{\Gamma}\right]=B D\left[\mathcal{E}_{\Gamma}\right]$, as we have seen in the proof of Proposition 4.1. Thus applying Theorem 3.11 to $\Gamma$, we have

Theorem 4.2 For a connected, infinite network $\Gamma=(V, E, r)$, the following conditions are equivalent:

(i) the effective resistance $R_{\Gamma}\left(=R_{\mathcal{E}_{\Gamma}}\right)$ is bounded,

(ii) $D\left[\mathcal{E}_{\Gamma}\right]=B D\left[\mathcal{E}_{\Gamma}\right]$, that is, any $u \in D\left[\mathcal{E}_{\Gamma}\right]$ is bounded.

Moreover if these are the cases, then the canonical map from the Royden boundary $\partial \mathcal{R}\left(V, \mathcal{E}_{\Gamma}\right)$ onto the Kuramochi boundary $\partial \bar{V}^{N}\left(\mathcal{E}_{\Gamma}\right)$ is homeomorphic.

Now we shall consider two conditions under which the Royden boundary of a connected, infinite network reduces to a single point.

Proposition 4.3 Let $\Gamma=\Gamma_{1} \times \Gamma_{2}$ be the cartesian product of connected, infinite networks $\Gamma_{1}=\left(V_{1}, E_{1}, r_{1}\right)$ and $\Gamma_{2}=\left(V_{2}, E_{2}, r_{2}\right)$. If $\Gamma_{2}$ satisfies the conditions in Proposition 4.1, then the Royden boundary of $\Gamma$ consists of a single point.

Proof. Let $f$ be a function in $B D\left[\mathcal{E}_{\Gamma}\right]$. We would like to show that $f(x, y)$ tends to a constant as $(x, y) \in V=V_{1} \times V_{2} \rightarrow \infty$. For a fixed $x \in V_{1}$, we have a function $f_{x}$ on $V_{2}$ defined by $f_{x}(y)=f(x, y)\left(y \in V_{2}\right)$. We decompose $f_{x}$ as $f_{x}=h_{x}+g_{x}$, where $h_{x} \in B H D\left[\mathcal{E}_{\Gamma_{2}}\right]$ and $g_{x} \in B D_{0}\left[\mathcal{E}_{\Gamma_{2}}\right]$. By the assumption, every $g_{x}$ vanishes at infinity.

Now for any pair of points $x, x^{\prime} \in V_{1}$, we take a path $\left\{x_{i}, i=0,1, \ldots, k\right\}$ in $\Gamma_{1}$ connecting $x\left(=x_{0}\right)$ and $x^{\prime}\left(=x_{k}\right)$, and then we have

$$
\begin{aligned}
\left|f_{x}(y)-f_{x^{\prime}}(y)\right| & \leq \sum_{i=0}^{k-1}\left|f\left(x_{i}, y\right)-f\left(x_{i+1}, y\right)\right| \\
& \leq\left(\sum_{i=0}^{k-1} \frac{\left|f\left(x_{i}, y\right)-f\left(x_{i+1}, y\right)\right|^{2}}{r_{1}\left(\left|x_{i} x_{i+1}\right|\right)}\right)^{1 / 2}\left(\sum_{i=0}^{k-1} r_{1}\left(\left|x_{i} x_{i+1}\right|\right)\right)^{1 / 2} .
\end{aligned}
$$


This shows that $\left|f_{x}(y)-f_{x^{\prime}}(y)\right|$ goes to zero as $y \in V_{2} \rightarrow \infty$ and hence so does $\left|h_{x}(y)-h_{x^{\prime}}(y)\right|$. Therefore we get $h_{x}=h_{x^{\prime}}$ for all $x, x^{\prime} \in V_{1}$. Moreover we see that $\mathcal{E}_{\Gamma_{2}}\left(h_{x}\right)=0$, that is, $h_{x}$ is equal to a constant $c$, because we have

$$
\sum_{x^{\prime} \in V_{1}} \mathcal{E}_{\Gamma_{2}}\left(h_{x}\right)=\sum_{x^{\prime} \in V_{1}} \mathcal{E}_{\Gamma_{2}}\left(h_{x^{\prime}}\right) \leq \sum_{x^{\prime} \in V_{1}} \mathcal{E}_{\Gamma_{2}}\left(f_{x^{\prime}}\right) \leq \mathcal{E}_{\Gamma}(f)<+\infty .
$$

Since $\left|g_{x}(y)\right| \leq M \mathcal{E}_{\Gamma_{2}}\left(g_{x}\right)=M \mathcal{E}_{\Gamma_{2}}\left(f_{x}\right)$ for some constant $M$ and all $y \in V_{2}$, and $\mathcal{E}_{\Gamma_{2}}\left(f_{x}\right)$ tends to zero as $x \in V_{1} \rightarrow \infty$, it follows that $f-c$ vanishes at infinity. This completes the proof of Proposition 4.3.

Proposition 4.4 Let $\Gamma=(V, E, r)$ be a connected, infinite network. Let $\left\{U_{n}\right\}$ and $\left\{V_{n}\right\}$ increasing sequences of finite subsets of $V$ such that $V_{n} \subset U_{n}$ and $\cup_{n} V_{n}=V$. Suppose that $\Gamma$ satisfies the conditions in Proposition 4.1 and that the finite subnetwork $\Gamma_{n}$ of $\Gamma$ generated by $A_{n}=U_{n} \backslash V_{n}$ is connected, and for infinitely many $n$, the effective resistance of $\Gamma_{n}$ is bounded by a constant independent of $n$. Then $\partial \mathcal{R}\left(V, \mathcal{E}_{\Gamma}\right)=\{\infty\}$. In particular, the effective resistance of $\Gamma$ is also bounded.

Proof. Since any function in $D_{0}\left[\mathcal{E}_{\Gamma}\right]$ vanishes at infinity, it is enough to show that a bounded harmonic function $h$ in $D\left[\mathcal{E}_{\Gamma}\right]$ is constant. Let $h_{n}$ be the restriction of $h$ to $A_{n}$. Then by the assumption,

$$
\sup _{x, y \in A_{n}}|h(x)-h(y)|^{2}=\sup _{x, y \in A_{n}}\left|h_{n}(x)-h_{n}(y)\right|^{2} \leq C \mathcal{E}_{\Gamma_{n}}\left(h_{n}\right)
$$

for some constant $C$ and infinitely many $n$. Since $\mathcal{E}_{\Gamma_{n}}\left(h_{n}\right)$ goes to zero as $n \rightarrow \infty$, it follows from the maximum principle that $h(x)$ tends to a constant as $x \in V \rightarrow \infty$, and indeed it is constant. This completes the proof of Proposition 4.4.

Vanishing theorems on $L^{2}$-harmonic forms on complete Riemannian manifolds have been investigated by many authors. As mentioned in Introduction, a connected, locally finite, infinite graph has no nonconstant harmonic functions of finite Dirichlet sums if it is quasi-isometric to a complete, noncompact Riemannian manifold such that the Ricci curvature is bounded from below, the volume of every unit ball is bounded away from zero, and it possesses no nontrivial $L^{2}$-harmonic one forms.

Now we consider a connected, locally finite, infinite graph $G=(V, E)$. There are certain cases where the compactifications $\mathcal{L}_{b}\left(V, d_{r}\right)$ relative to weight functions $r: E \rightarrow[0,+\infty)$ play important roles in geometries of $G$. Let $d_{G}$ the graph distance on $V$. Fix a vertex $o$ of $V$ and set $S(n)=\{x \in$ $\left.V \mid d_{G}(X, o)=n\right\}$ and $E(n)=\{|x y| \in E \mid x, y \in S(n)$ or $x \in S(n), y \in$ $S(n-1)\}$. 
An extended real number $e(G)$ is assigned to $G$ as follows:

$$
e(G)=\limsup _{n \rightarrow \infty} \frac{1}{n} \log \sharp E(n) \in[0,+\infty] .
$$

Let us consider a special family of weights on $E,\left\{r_{\delta} \mid \delta>0\right\}$, defined by $r_{\delta}(x, y)=e^{-\delta n}$ for $|x y| \in E(n)(n=1,2,3, \ldots)$. In what follows, $d_{\delta}, \Gamma_{\delta}$ and $\mathcal{E}_{\delta}$ stand for the distance $d_{r_{\delta}}$, the network $\left(V, E, r_{\delta}\right)$ and the Dirichlet sum $\mathcal{E}_{\Gamma_{\delta}}$, respectively. Since the diameter of the metric space $\left(V, d_{\delta}\right)$ is bounded and the effective resistance of $\mathcal{E}_{\delta}$ is also bounded, the identity map of $V$ induces a homeomorphism between the Royden boundary $\partial \mathcal{R}\left(V, \mathcal{E}_{\delta}\right)$ and the Kuramochi boundary $\partial \bar{V}^{N}\left(\mathcal{E}_{\delta}\right)$ of $\Gamma_{\delta}$.

Lemma 4.5 (i) Any function in $D\left[\mathcal{E}_{\delta}\right]$ is Lipschitz continuous with respect to the distance $d_{\delta / 2}$, so that the identity map of $V$ induces a continuous map from $\partial \mathcal{C}\left(V, d_{\delta / 2}\right)$ onto $\partial \mathcal{R}\left(V, \mathcal{E}_{\delta}\right)$.

(ii) If $e(G)$ is finite, then for positive constants $\delta$ and $\eta$ with $\eta<2 \delta-$ $e(G)$, any Lipschitz continuous function with respect to the distance $d_{\delta}$ belongs to $D\left[\mathcal{E}_{\eta}\right]$, so that the identity map of $V$ induces a continuous map from $\partial \mathcal{R}\left(V, \mathcal{E}_{\eta}\right)$ onto $\partial \mathcal{L}_{b}\left(V, d_{\delta}\right)$.

Proof. For $u \in D\left[\mathcal{E}_{\delta}\right], \mathcal{E}_{\delta}(u)=\sum_{n=1}^{\infty} e^{\delta n}\left(\sum_{e \in E(n)}|d u(e)|^{2}\right)$ is finite, and hence $\sup _{e \in E(n)}|d u(e)|^{2}$ is bounded by $b^{2} e^{-\delta n}$, where $b$ is a positive constant. Given any pair of points $x, y \in V$, let $C=\left\{x=x_{0}, x_{1}, \ldots, x_{k}=y\right\}$ be a path connecting $x$ to $y$. Then we have

$$
|u(x)-u(y)| \leq \sum_{i=0}^{k-1}\left|u\left(x_{i}\right)-u\left(x_{i+1}\right)\right| \leq b L_{r_{\delta / 2}}(C),
$$

and hence $|u(x)-u(y)| \leq b d_{\delta / 2}(x, y)$. In this way, the first assertion is verified.

Let $v$ be a function satisfying $|v(x)-v(y)| \leq b d_{\delta}(x, y)$ for some positive constant $b$ and all $x, y \in V$. Then, since $|d v(e)|^{2} \leq b^{2} e^{2 \delta n}$ for $e \in E(n)$, we get

$$
\mathcal{E}_{\eta}(v)=\sum_{n=1}^{\infty} e^{\eta n} \sum_{e \in E(n)}|d v(e)|^{2} \leq b^{2} \sum_{n=1}^{\infty} e^{(\eta-2 \delta) n} \sharp E(n),
$$

which is finite if $0<\eta<2 \delta-e(G)$. This proves the second assertion.

Now we assume that a graph $G=(V, E)$ under consideration is hyperbolic in the sense of Gromov, that is, the geodesic graph $|G|$ is hyperbolic in the sense of Gromov. Then it is known (cf. [23], [15]) that there exists $\delta_{0}(G) \in(0,+\infty]$ such that for any positive constant $\delta<\delta_{0}(G)$, the 
completion of $d_{\delta}$ coincides with the compactification $\mathcal{L}_{b}\left(V, d_{\delta}\right)$ and moreover $\partial \mathcal{L}_{b}\left(V, d_{\delta}\right)$ is homeomorphic to the Gromov boundary $\partial_{H} G$ of $G$. Then the following can be deduced from Lemma 4.5.

Proposition 4.6 Let $G=(V, E)$ be a connected, locally finite, infinite graph which is hyperbolic in the sense of Gromov. Suppose that $e(G)<$ $2 \delta_{0}(G)$. Then for any $0<\eta<2 \delta_{0}(G)-e(G), \partial \mathcal{R}\left(V, \mathcal{E}_{\eta}\right)$ is homeomorphic to $\partial_{H} G$; in particular, the identity map of $V$ extends continuously from the Royden boundary of $G$ onto the Gromov boundary of $G$.

Now we are given a metric space $\left(Y, d_{Y}\right)$ and a compactification $\tilde{Y}$ of $Y$ which is separated by the distance $d_{Y}$, i.e., $\liminf _{x \rightarrow p, y \rightarrow q} d_{Y}(x, y)>0$ for all $x \in Y \rightarrow p \in \partial Y$ and $y \in Y \rightarrow q \in \partial Y$ if $p \neq q$. We define the Dirichlet sum of a map $\phi$ from $\Gamma=(V, E, r)$ to $Y$ by

$$
\mathcal{E}_{\Gamma ; Y}(\phi)=\frac{1}{2} \sum_{x \sim y} \frac{d_{X}(\phi(x), \phi(y))^{2}}{r(|x y|)} .
$$

If $\phi$ possesses finite Dirichlet sum, then it extends to a continuous map from the Royden compactification of $\Gamma$ onto the closure of the image $\phi(V)$ in $\tilde{Y}$. Indeed, for any bounded Lipschitz function $f$ on $Y$, the composition $f \circ \phi$ belongs to $B D\left[\mathcal{E}_{\Gamma}\right]$.

For instance, let $r$ be a weight on $E$ that is square integrable, i.e., $\sum_{e \in E} r(e)^{2}$ is finite. Then considering the identity map $I$ of $V$ as a map from the graph $G=(V, E)$ onto the metric space $\left(V, d_{r}\right)$, the Dirichlet sum of $I$ is just equal to $\sum_{e \in E} r(e)^{2}$, so that $I$ extends to a continuous map from the Royden compactification of $G$ onto $\mathcal{L}_{b}\left(V, d_{r}\right)$ which sends the Royden boundary onto $\partial \mathcal{L}_{b}\left(V, d_{r}\right)$.

Here we refer to a result due to Cartwright and Woess [11] (see also [37, Chap. IV $\S 6])$. Let $G=(V, E)$ be, as in Theorem 1.15, a connected, locally finite, infinite graph uniformly embedded in the unit disk $\Omega$ of the complex plane. We assume that $\Omega$ is endowed with the Euclidean distance $d_{E}$. Let $\iota$ be the inclusion of $V$ into the metric space $\left(\Omega, d_{E}\right)$ and $V^{\prime}$ the accumulation points of $V$ on the unit circle. Then it is proved in [11] that the Dirichlet sum of $\iota$ is finite if $G$ satisfies a strong isoperimetric inequality, and as a consequence, for every Lipschitz continuous function $\phi$ on the Euclidean closure $\bar{\Omega}$ of $\Omega$, there exists a unique function $h$ on $V \cup V^{\prime}$ such that $h$ coincides with $\phi$ on $V^{\prime}$ and the restriction of $h$ to $V$ is in $H D\left[\mathcal{E}_{G}\right]$. We recall that $G$ satisfies a strong isoperimetric inequality if and only if $\mathcal{E}_{G}^{0}$ has a positive spectral gap $\lambda_{0}$ in $L^{2}\left(V, \mu^{c}\right)$, that is, $\sum_{x \in V} u(x)^{2} \leq \lambda_{0}{ }^{-1} \mathcal{E}_{G}^{0}(u)$ for all $u \in D_{0}\left[\mathcal{E}_{G}\right]$ (cf. e.g., [37, Theorem (4.27)]). If these are the cases, the minimal Green 
function $g_{G}^{0}$ of $G$ is bounded from above by $2 / \lambda_{0}$ (cf. Remark 3.1 (ii)), so that the conditions of Proposition 4.1 are satisfied. Therefore $\iota$ as above extends to a continuous map of the harmonic boundary $\Delta(G)$ of $G$ onto $V^{\prime}$, and thus if $V^{\prime}$ has more than one point, then $G$ has nonconstant harmonic functions of finite Dirichlet sums. We refer the reader to [3] and [4] for related results.

Now we consider a finitely generated, infinite, properly discontinuous subgroup $\Pi$ of isometries of a proper geodesic space $\left(Y, d_{Y}\right)$. Fix a point $o$ of $Y$ and let $\psi: \Pi \rightarrow Y$ be a map from $\Pi$ into $Y$ defined by $\psi(g)=$ $g^{-1}(o), g \in \Pi$. We take a finite generating set $S$ of $\Pi$ with $S=S^{-1}$ and consider the Cayley graph $G_{\Pi}=\left(\Pi, E_{S}\right)$. First we carry out "conformal" changes of the metric of $Y$ as in the case of graphs. For a positive continuous function $w$ on $Y$, the $w$-length $L_{w}(c)$ of an arc-length parametrized curve $c:[a, b] \rightarrow\left(Y, d_{Y}\right)$ is given by $L_{w}(c)=\int_{a}^{b} w(c(t)) d t$. Then we define a distance $d_{w}(x, y)$ between two points $x$ and $y$ of $Y$ by the infimum of $L_{w}(c)$ where $c$ ranges over all arc-length parametrized curves joining $x$ and $y$. As in the case of graphs, we are interested in the case when $w_{\varepsilon}=\exp \left(-\varepsilon d_{Y}(*, o)\right)$, where $\varepsilon$ is a positive constant. Then the Dirichlet sum of the map $\psi$ from $G_{\Pi}$ into the metric space $Y_{\varepsilon}=\left(Y, d_{w_{\varepsilon}}\right)$ is given by

$$
\mathcal{E}_{G_{\Pi} ; Y_{\varepsilon}}(\psi)=\frac{1}{2} \sum_{g \in \Pi, a \in S} d_{w_{\varepsilon}}(\psi(g), \psi(a g))^{2} .
$$

Proposition 4.7 Suppose that the completion $\bar{Y}^{\varepsilon}$ of the metric space $\left(Y, d_{w_{\varepsilon}}\right)$ is compact, and suppose that the critical exponent of $\Pi$ defined by

$$
\bar{e}(\Pi)=\inf \left\{s>0 \mid \sum_{g \in \Pi} \exp \left(-s d_{Y}(o, g(o))\right)<+\infty\right\}
$$

is finite and $2 \varepsilon>\bar{e}(\Pi)$, then $\psi$ extends to a continuous map of the Royden boundary of the Cayley graph $G_{\Pi}$ onto the intersection of the closure of the orbit $\Pi(o)$ in $\bar{Y}^{\varepsilon}$ and the boundary of $\bar{Y}^{\varepsilon}$.

This is an extension of Theorem 2 with $p=2$ in [8], where the space of functions of finite Dirichlet sums of order $p(>1)$ are studied and the theorem is concerning isometric actions on a proper CAT $(-1)$ space. The arguments there are valid for a proof of Proposition 4.7 and further it is possible to show the proposition for functions of finite Dirichlet sums of order $p>1$. We refer the reader to [2] for some related results to Proposition 4.3, [7], [19] for those to Proposition 4.6, and [25] for some extensions of Proposition 4.1, Theorem 4.2 and Proposition 4.7 to the case of Dirichlet sums of order $p>1$. 


\section{Gromov-Hausdorff and variational convergence of networks}

In this section, we study Gromov-Hausdorff and variational convergence of networks and exhibit some examples.

5.1 We begin with verifying that a connected, infinite network $\Gamma=(V, E, r)$ is the limit of an exhausting family of connected subnetworks of $\Gamma$. Given a subset $W$ of $V$, we denote by $\Gamma_{W}=\left(W, E_{W}, r_{W}\right)$ the subnetwork generated by $W$ and write $\mathcal{E}_{W}$ and $R_{W}$ for $\mathcal{E}_{\Gamma_{W}}$ and $R_{\mathcal{E}_{W}}$ respectively. It follows from Lemma 2.5 that for subsets $W$ and $U$ with $W \subset U, R_{\mathcal{E}_{\Gamma}}(x, y) \leq R_{U}(x, y) \leq$ $R_{W}(x, y)$ for all $x, y \in W$. Let $W$ be a finite subset of $V$ and take an increasing sequences of finite subsets $V_{i}$ of $V$ such that $W \subset V_{i}, V=\cup_{i} V_{i}$ and $\Gamma_{V_{i}}$ is connected. The family of the traces $\mathcal{E}_{V_{i}: W}^{*}$ of $\mathcal{E}_{V_{i}}$ on $W$ satisfies $\mathcal{E}_{V_{i} ; W}^{*}(u) \leq \mathcal{E}_{V_{j} ; W}^{*}(u) \leq \mathcal{E}_{\Gamma ; W}^{*}(u)$ for $i<j$ and $u \in \ell(W)$. Given $u \in \ell(W)$, let $u_{i}$ be a unique function on $V_{i}$ such that $u_{i}=u$ on $W, \mathcal{E}_{V_{i}}\left(u_{i}, v\right)=0$ for all $v \in \ell\left(V_{i}\right)$ vanishing on $W$, and $\mathcal{E}_{V_{i}}\left(u_{i}\right)=\mathcal{E}_{V_{i} ; W}^{*}(u)$ (cf. Lemma 1.6). Then the maximum principle implies that $\min _{W} u \leq u_{i} \leq \max _{W} u$ on $V_{i}$. Then passing to a subsequence, we assume that $u_{i}$ converges pointwise to a function $u_{\infty}$ on $V$ as $i \rightarrow \infty$. For a fixed $j$, we have

$$
\mathcal{E}_{V_{j}}\left(u_{\infty \mid V_{j}}\right)=\lim _{i \rightarrow \infty} \mathcal{E}_{V_{j}}\left(u_{i \mid V_{j}}\right) \leq \lim _{i \rightarrow \infty} \mathcal{E}_{V_{i}}\left(u_{i}\right) \leq \mathcal{E}_{\Gamma ; W}^{*}(u) .
$$

Therefore letting $j$ go to infinity, we get $\mathcal{E}_{\Gamma}\left(u_{\infty}\right) \leq \mathcal{E}_{\Gamma ; W}^{*}(u)$. Since $u_{\infty}=u$ on $W$, we see that $\mathcal{E}_{\Gamma}\left(u_{\infty}\right)=\mathcal{E}_{\Gamma ; W}^{*}(u)$, that is, $u_{\infty}$ is a unique minimizer of $\mathcal{E}_{\Gamma}$ in the family $\left\{f \in D\left[\mathcal{E}_{\Gamma}\right] \mid f(x)=u(x), x \in W\right\}$. Moreover it follows from the above argument that $\lim _{i \rightarrow \infty} \mathcal{E}_{V_{i} ; W}^{*}(u)=\lim _{i \rightarrow \infty} \mathcal{E}_{V_{i}}\left(u_{i}\right)=\mathcal{E}_{\Gamma ; W}^{*}(u)$. This holds for all $u \in \ell(W)$, which shows that $\lim _{i \rightarrow \infty} R_{V_{i}}(x, y)=R_{\Gamma}(x, y)$ for all $x, y$ of $W$, and hence $V$.

Now we prove the following

Theorem 5.1 Let $(X, R)$ and $\left\{\Gamma_{n}=\left(V_{n}, E_{n}, r_{n}, R_{n}\right)\right\}$ be a separable metric space and a sequence of connected, finite networks with the resistance metrics $R_{n}$. Let $p$ and $p_{n}$ be points of $X$ and $V_{n}$ respectively. Suppose that there exist sequences of positive numbers $\left\{\rho_{n}\right\}$ and $\left\{\varepsilon_{n}\right\}$ with $\lim _{n \rightarrow \infty} \rho_{n}=+\infty$ and $\lim _{n \rightarrow \infty} \varepsilon_{n}=0$, and a sequence of maps $f_{n}: B_{\rho_{n}}\left(p_{n}\right) \rightarrow X$ from the metric ball $B_{\rho_{n}}\left(p_{n}\right)$ of $V_{n}$ around $p_{n}$ of radius $\rho_{n}$ to $X$ such that $f_{n}\left(p_{n}\right)=p$, $\left|R_{n}(x, y)-R\left(f_{n}(x), f_{n}(y)\right)\right| \leq \varepsilon_{n}$ for all $x, y \in B_{\rho_{n}}\left(p_{n}\right)$, and furthermore any finite subset $W$ of $X$ is included in the $\varepsilon_{n}$-neighborhood of the image $f_{n}\left(B_{\rho_{n}}\left(p_{n}\right)\right)$ for all $n$ large enough. Then the distance $R$ of $X$ is the resistance metric on $X$ associated to a resistance form $(\mathcal{E}, D[\mathcal{E}])$. 
Proof. Given a finite subset $W=\left\{x_{1}, \ldots, x_{k}\right\}$ of $X$, we take a finite subset $W_{n}=\left\{x_{1 ; n}, \ldots, x_{k ; n}\right\}$ of $V_{n}$ in such a way that $R\left(f_{n}\left(x_{i ; n}\right), x_{i}\right)$ tends to 0 as $n \rightarrow \infty$ for all $i=1, \ldots, k$. Then from (1.1), (1.2), (1.3) and (1.5) in section 1 , we can deduce that there exists a unique resistance form $\mathcal{E}_{W}^{*}$ on $W$, the resistance metric associated to which is just the metric $R$ restricted to $W$. Moreover we observe that for any finite subsets $W_{1}, W_{2}$ of $X, \mathcal{E}_{W_{1}}^{*}$ is the trace of $\mathcal{E}_{W_{2}}^{*}$ on $W_{1}$ if $W_{1} \subset W_{2}$; in particular, $\mathcal{E}_{W_{1}}^{*}\left(u_{\mid W_{1}}\right) \leq \mathcal{E}_{W_{2}}^{*}(u)$ for any $u \in \ell\left(W_{2}\right)$. Let us now choose an increasing sequence of finite subsets $W_{i}$ of $X$ in such a way that $W_{*}=\cup_{i} W_{i}$ is dense in $X$, and define a form $\mathcal{E}$ on a subspace $D[\mathcal{E}]$ of $\ell\left(W_{*}\right)$ by

$$
D[\mathcal{E}]=\left\{u \in \ell\left(W_{*}\right) \mid \lim _{i \rightarrow \infty} \mathcal{E}_{W_{i}}^{*}\left(u_{\mid W_{i}}\right)<+\infty\right\} ; \mathcal{E}(u)=\lim _{i \rightarrow \infty} \mathcal{E}_{W_{i}}^{*}\left(u_{\mid W_{i}}\right) .
$$

Then it is easy to see that the effective resistance $R_{\mathcal{E}}(x, y)$ between two points $x$ and $y$ of $W_{*}$, that is, $R_{\mathcal{E}}(x, y)=\sup \left\{|u(x)-u(y)|^{2} / \mathcal{E}(u) \mid u \in\right.$ $D[\mathcal{E}], \mathcal{E}(u)>0\}$ is equal to $R(x, y)$. Thus every function $u \in D[\mathcal{E}]$ uniquely extends to a continuous function on $X$ and the form is considered to be defined on a subspace of $C(X, R)$. It is evident that $\mathcal{E}$ verifies the conditions [H-2], [H-4] and [H-7]. This completes the proof of Theorem 5.1.

Corollary 5.2 Let $(X, \mathcal{E})$ and $\Gamma_{n}=\left(V_{n}, E_{n}, r_{n}\right)$ be as in Theorem 5.1. Then one has

$$
\limsup _{n \rightarrow \infty} \lambda_{1}\left(\mathcal{E}_{\Gamma_{n}} ; \mu_{V_{n}}^{c}\right) \leq \lambda_{1}^{*}\left(\mathcal{E} ; \mu_{X}^{c}\right) \text {. }
$$

Proof. For a finite subset $W$ of $X$, let $W_{n}$ be a finite subset of $V_{n}$ as in the proof of Theorem 5.1. Then as $n \rightarrow \infty$, the first nonzero eigenvalue of $\mathcal{E}_{W_{n}}^{*}$, $\lambda_{1}\left(\mu_{W_{n}}^{c}\right)$, converges to that of $\mathcal{E}_{W}^{*}, \lambda_{1}\left(\mu_{W}^{c}\right)$. Therefore we have

$$
\limsup _{n \rightarrow \infty} \lambda_{1}\left(\mu_{V_{n}}^{c}\right) \leq \limsup _{n \rightarrow \infty} \lambda_{1}\left(\mu_{W_{n}}^{c}\right)=\lambda_{1}\left(\mu_{W}^{c}\right) .
$$

This holds for all finite subsets $W$ of $X$, and so does for $\lambda_{1}^{*}\left(\mu_{X}^{c}\right)$. This completes the proof of the corollary.

Definition 5.1 Given two metric spaces $X, Y$ and a positive number $\varepsilon$, a (not necessarily continuous) map $f: X \rightarrow Y$ is called an $\varepsilon$-Hausdorff approximation if

$$
\sup _{x_{1}, x_{2} \in X}\left|d_{Y}\left(f\left(x_{1}\right), f\left(x_{2}\right)\right)-d_{X}\left(x_{1}, x_{2}\right)\right|<\varepsilon
$$

and the image $f(X)$ of $f$ is an $\varepsilon$-net in $Y$, i.e., $d_{Y}(y, f(X))<\varepsilon$ for every $y \in Y$. We say that a sequence of compact metric spaces $\left\{X_{n}\right\}$ converges to a compact metric space $X$ in the Gromov-Hausdorff sense if there exist $\varepsilon_{n}$-Hausdorff approximations $f_{n}: X_{n} \rightarrow X$ with $\lim _{n \rightarrow \infty} \varepsilon_{n}=0$. 
We remark that every compact geodesic space can be obtained as a Gromov-Hausdorff limit of compact geodesic graphs endowed with their geodesic distances (cf. [9, Proposition 7.5.5]).

Definition 5.2 A pointed metric space $(X, p)$ is a pair of a metric space $X$ and a point $p \in X$. We say that a sequence $\left\{\left(X_{n}, p_{n}\right)\right\}$ of pointed metric spaces converges to a pointed metric space $(X, p)$ in the Gromov-Hausdorff sense if the following holds. For every $\rho>0$ and $\varepsilon>0$, there exists a positive integer $n_{0}$ such that for any $n>n_{0}$, there is a map $f$ from the ball $B_{\rho}\left(p_{n}\right)$ around $p_{n}$ with radius $\rho$ in $X_{n}$ to $X$ satisfying the following properties:

(i) $f\left(p_{n}\right)=p$;

(ii) $\sup \left\{\left|d_{X}\left(f\left(x_{1}\right), f\left(x_{2}\right)\right)-d_{X_{n}}\left(x_{1}, x_{2}\right)\right| \mid x_{1}, x_{2} \in B_{\rho}\left(p_{n}\right)\right\}<\varepsilon$;

(iii) the $\varepsilon$-neighborhood of the set $f\left(B_{\rho}\left(p_{n}\right)\right)$ includes the ball $B_{\rho-\varepsilon}(p)$ centered at $p$ of radius $\rho-\varepsilon$.

Now we prove the following

Theorem 5.3 Let $\left\{\Gamma_{n}=\left(V_{n}, E_{n}, r_{n}\right)\right\}$ be a sequence of connected, locally finite networks endowed with the geodesic distance $d_{r_{n}}$, and let $\Gamma=(V, E, r)$ be a connected, locally finite, infinite network with the geodesic distance $d_{r}$ such that for each vertex $p$ and each positive number $\rho$, the metric ball $B_{\rho}(p)$ in $\left(V, d_{r}\right)$ consists of finite vertices. Suppose that the pointed metric space $\left(V_{n}, p_{n}, d_{r_{n}}\right)$ converges, as $n \rightarrow \infty$, to the pointed metric space $\left(V, p, d_{r}\right)$ in the Gromov-Hausdorff sense, and that for any large $\rho$, there exist positive constants $a_{\rho}, b_{\rho}$ and a positive integer $n_{\rho}$ such that $a_{\rho} \leq r_{n} \leq b_{\rho}$ on the geodesic ball $B_{\rho}\left(p_{n}\right)$ for all $n \geq n_{\rho}$. Then there exist a subsequence $\left\{\Gamma_{m}\right\}$ and a resistance form $\mathcal{E}$ on $V$ such that the pointed metric space $\left(V_{m}, p_{m}, R_{\Gamma_{m}}\right)$ with the effective resistance $R_{\Gamma_{m}}$ relative to $\left(\mathcal{E}_{\Gamma_{m}}, D\left[\mathcal{E}_{\Gamma_{m}}\right]\right)$ converges, as $m \rightarrow \infty$, to the pointed metric space $\left(V, p, R_{\mathcal{E}}\right)$ with the resistance metric $R_{\mathcal{E}}$ relative to the resistance form $\mathcal{E}$ in the sense of Theorem 5.1. Moreover the from $\mathcal{E}$ satisfies that $D_{0}\left[\mathcal{E}_{\Gamma}^{0}\right] \subset D[\mathcal{E}] \subset D\left[\mathcal{E}_{\Gamma}\right], \mathcal{E}(u) \geq \mathcal{E}_{\Gamma}(u)$ for all $u \in D[\mathcal{E}]$, and $\mathcal{E}(u, v)=\mathcal{E}_{\Gamma}(u, v)$ for all $u \in D[\mathcal{E}]$ and $v \in D_{0}\left[\mathcal{E}_{\Gamma}^{0}\right]$.

Proof. We take sequences of positive numbers $\left\{\rho_{n}\right\}$ and $\left\{\varepsilon_{n}\right\}$ respectively with $\lim _{n \rightarrow \infty} \rho_{n}=+\infty$ and $\lim _{n \rightarrow \infty} \varepsilon_{n}=0$ in such a way that there exists a sequence of approximating maps $f_{n}$ of the metric balls $B_{\rho_{n}}\left(p_{n}\right)$ of $\left(V_{n}, d_{r_{n}}\right)$ to $V$ satisfying $f_{n}\left(p_{n}\right)=p, \sup \left\{\left|d_{r}\left(f_{n}\left(x_{1}\right), f_{n}\left(x_{2}\right)\right)-d_{r_{n}}\left(x_{1}, x_{2}\right)\right| \mid x_{1}, x_{2} \in\right.$ $\left.B_{\rho_{n}}\left(p_{n}\right)\right\}<\varepsilon_{n}$, and the $\varepsilon_{n}$-neighborhood of the set $f_{n}\left(B_{\rho_{n}}\left(p_{n}\right)\right)$ includes the ball $B_{\rho_{n}-\varepsilon_{n}}(p)$ centered at $p$ of radius $\rho_{n}-\varepsilon_{n}$ in $\left(V, d_{r}\right)$. In addition, we choose approximating maps $h_{n}: B_{\rho_{n} / 2}(p) \rightarrow B_{\rho_{n}}\left(p_{n}\right)$ such that $d_{r}\left(f_{n}\left(h_{n}(x)\right), x\right) \leq$ $2 \varepsilon_{n}$ for all $x \in B_{\rho_{n} / 2}(p)$. Then in view of (1.6), we are able to choose a subsequence $\left\{\Gamma_{m}\right\}$ in such a way that for any $x, y \in V$ with $x \neq y$, 
the effective resistance $R_{\Gamma_{m}}\left(h_{m}(x), h_{m}(y)\right)$ between $h_{m}(x)$ and $h_{m}(y)$ in $V_{m}$ converges to a positive number $R(x, y)$ as $m \rightarrow \infty$. In fact, as we have seen in Theorem 5.1, the limit $R(x, y)$ is equal to the effective resistance $R_{\mathcal{E}}(x, y)$ between $x$ and $y$ in $V$ with respect to a resistance form $\mathcal{E}$ on $V$; moreover if we denote by $\mathcal{E}_{m ; \rho}^{*}\left(\right.$ resp. $\left.\mathcal{E}_{\rho}^{*}\right)$ the trace of $\mathcal{E}_{\Gamma_{m}}$ on the metric ball $B_{\rho}\left(p_{m}\right)$ of $\left(V_{m}, d_{r_{m}}\right)$ (resp. the trace of $\mathcal{E}$ on the metric ball $B_{\rho}(p)$ of $\left(V, d_{r}\right)$ ), then we have

$$
\mathcal{E}_{\rho}^{*}\left(\chi_{x}, \chi_{y}\right)=\lim _{m \rightarrow \infty} \mathcal{E}_{m ; \rho}^{*}\left(\chi_{h_{m}(x)}, \chi_{h_{m}(y)}\right)
$$

for all $x, y \in B_{\rho}(p)$. Since $0<a_{\rho} \leq r_{m} \leq b_{\rho}$, we see that for any $x, y \in$ $V$ with $x \neq y$ and for all $\rho$ and $m$ large enough, $\mathcal{E}_{m ; \rho}^{*}\left(\chi_{h_{m}(x)}, \chi_{h_{m}(y)}\right)=$ $\mathcal{E}_{\Gamma_{m}}\left(\chi_{h_{m}(x)}, \chi_{h_{m}(y)}\right)$, which is equal to $-r_{m}\left(\left|h_{m}(x) h_{m}(y)\right|\right)^{-1}$ if $x \sim y$, and equal to 0 otherwise; thus $\mathcal{E}_{\rho}^{*}\left(\chi_{x}, \chi_{y}\right)=\lim _{m \rightarrow \infty}-r_{m}\left(\left|h_{m}(x) h_{m}(y)\right|\right)^{-1}=$ $-r(|x y|)^{-1}$ if $x \sim y$ and $\mathcal{E}_{\rho}^{*}\left(\chi_{x}, \chi_{y}\right)=0$ otherwise. In the same way, we get $\mathcal{E}_{\rho}^{*}\left(\chi_{x}\right)=\sum_{z \sim x} r(|x z|)^{-1}$. Since $\mathcal{E}\left(\chi_{x}\right)=\lim _{\rho \rightarrow \infty} \mathcal{E}_{\rho}^{*}\left(\chi_{x}\right)$, it follows that $\chi_{x} \in D[\mathcal{E}]$ and for all $x, y \in V$,

$$
\mathcal{E}\left(\chi_{x}, \chi_{y}\right)=\left\{\begin{array}{cl}
\sum_{z \sim x} r(|x z|)^{-1} & \text { for } x=y \\
-r(|x y|)^{-1} & \text { for } x \sim y \\
0 & \text { otherwise }
\end{array}\right.
$$

As a consequence, for any finitely supported function $u$ on $V$, we have $\mathcal{E}(u)=$ $\mathcal{E}_{\Gamma}(u)=\frac{1}{2} \sum_{x \sim y}|u(x)-u(y)|^{2} / r(|x y|)$. Moreover for any function $v \in \ell(V)$, we have

$$
\begin{aligned}
\mathcal{E}_{\rho}^{*}\left(v_{\mid B_{\rho}(p)}\right) & =\lim _{m \rightarrow \infty} \mathcal{E}_{m ; \rho}^{*}\left(\left(v \circ f_{m}\right)_{\mid B_{\rho}\left(p_{m}\right)}\right) \\
\sum_{x, y \in B_{\rho}(p): x \sim y} \frac{|v(x)-v(y)|^{2}}{r(|x y|)} & =\lim _{m \rightarrow \infty} \sum_{x, y \in B_{\rho}\left(p_{m}\right): x \sim y} \frac{\left|v \circ f_{m}(x)-v \circ f_{m}(y)\right|^{2}}{r_{m}(|x y|)}
\end{aligned}
$$

and

$$
\frac{1}{2} \sum_{x, y \in B_{\rho}\left(p_{m}\right): x \sim y} \frac{\left|v \circ f_{m}(x)-v \circ f_{m}(y)\right|^{2}}{r_{m}(|x y|)} \leq \mathcal{E}_{m ; \rho}^{*}\left(\left(v \circ f_{m}\right)_{\mid B_{\rho}\left(p_{m}\right)}\right),
$$

and hence we get

$$
\frac{1}{2} \sum_{x, y \in B_{\rho}(p): x \sim y} \frac{|v(x)-v(y)|^{2}}{r(|x y|)} \leq \mathcal{E}_{\rho}^{*}\left(v_{\mid B_{\rho}(p)}\right) .
$$

Letting $\rho \rightarrow \infty$, we arrive at

$$
\mathcal{E}_{\Gamma}(v) \leq \mathcal{E}(v) \leq+\infty, \quad v \in \ell(V) .
$$

In the same way, we can show that for $u \in D[\mathcal{E}]$ and finitely supported functions $v$ on $V, \mathcal{E}(u, v)=\mathcal{E}_{\Gamma}(u, v)$. This completes the proof of Theorem 5.3. 
Remark 5.1 (i) The limit form $\mathcal{E}$ of Theorem 5.3 does not in general coincide with the Dirichlet sum $\mathcal{E}_{\Gamma}$ of the network $\Gamma$. See Remark 7.1 (i).

(ii) Given positive numbers $a, b$ and $c$, we consider a set of locally finite, connected networks $\Gamma=(V, E, r)$ such that $a \leq r \leq b$ on $E$ and the degree of any point of $V$ is bounded from above by $c$. Then the set of pointed metric spaces $\left(V, p, d_{r}\right)$ is compact with respect to the topology of the Gromov-Hausdorff convergence.

\subsection{We begin with}

Definition 5.3 Given a compact separable Hausdorff space $Y$, a sequence of such spaces $X_{n}$, and a sequence of maps $f_{n}: X_{n} \rightarrow Y$, we say that a sequence of functions $u_{n} \in C\left(X_{n}\right)$ uniformly converges to a function $u \in$ $C(Y)\left(\right.$ via $\left.f_{n}\right)$ if $\lim _{n \rightarrow \infty} \sup _{X_{n}}\left|u \circ f_{n}-u_{n}\right|=0$. Let $\mathcal{F}: C(Y) \rightarrow[0,+\infty]$ and $\mathcal{F}_{n}: C\left(X_{n}\right) \rightarrow[0,+\infty]$ be lower semi-continuous functionals on $C(Y)$ and $C\left(X_{n}\right)$ respectively. We say that $\mathcal{F}_{n} \Gamma$-converges to $\mathcal{F}$ if the following conditions are satisfied: (i) if a sequence of functions $u_{n} \in C\left(X_{n}\right)$ uniformly converges to a function $u \in C(Y)$, then we have

$$
\mathcal{F}(u) \leq \liminf _{n \rightarrow \infty} \mathcal{F}_{n}\left(u_{n}\right)(\leq+\infty)
$$

(ii) for any $u \in C(Y)$, there exists a sequence of functions $u_{n} \in C\left(X_{n}\right)$ such that $u_{n}$ uniformly converges to $u$ and

$$
\limsup _{n \rightarrow \infty} \mathcal{F}_{n}\left(u_{n}\right) \leq \mathcal{F}(u)(\leq+\infty)
$$

The following is a basic fact on this variational convergence.

Theorem 5.4 Let $Y$ and $\left\{X_{n}\right\}$ be respectively a compact separable Hausdorff space and a sequence of such spaces. Given a sequence of maps $f_{n}$ : $X_{n} \rightarrow Y$ and a sequence of lower semi-continuous functionals $\mathcal{F}_{n}: C\left(X_{n}\right) \rightarrow$ $[0,+\infty]$, there exists a subsequence, $\left\{X_{m}\right\}$, and a lower semi-continuous functional $\mathcal{F}: C(Y) \rightarrow[0,+\infty]$ such that $\mathcal{F}_{m} \Gamma$-converges to $\mathcal{F}$ as $m \rightarrow \infty$.

Proof. Using the idea of De Giorgi's $\Gamma$-convergence (cf. e.g., [16]), we introduce a functional on $C(Y)$ as follows: Let $\mathcal{B}=\left\{O_{i}\right\}$ be a countable basis of $C(Y)$ such that $O_{i}$ is totally bounded. Given $O_{i}$ and a positive integer $k$, let

$$
O_{i, k ; n}=\left\{v \in C\left(X_{n}\right)\left|\sup _{X_{n}}\right| u \circ f_{n}-v \mid<1 / k \text { for some } u \in O_{i}\right\},
$$

and

$$
E_{i, k ; n}=\inf \left\{\mathcal{F}_{n}(v) \mid v \in O_{i, k ; n}\right\}(\leq+\infty)
$$


Then passing to a subsequence, $\left\{X_{m}\right\}$, we may assume that for any $O_{i}$ and every $k, E_{i, k ; m}$ tends to an extended number $E_{i, k} \in[0,+\infty]$ as $m \rightarrow \infty$, and thus we are able to obtain a lower semi-continuous functional $\mathcal{F}: C(Y) \rightarrow$ $[0,+\infty]$ defined by

$$
\mathcal{F}(u)=\sup \left\{E_{i, k} \mid u \in O_{i}, k>0\right\}, \quad u \in C(Y),
$$

to which $\mathcal{F}_{m} \Gamma$-converges as $m \rightarrow \infty$.

Let $\mathcal{F}_{n}: C\left(X_{n}\right) \rightarrow[0,+\infty]$ and $\mathcal{F}: C(Y) \rightarrow[0,+\infty]$ be as in Theorem 5.4. If $\mathcal{F}_{n}$ is induced from a quadratic form $\mathcal{E}_{n}$ on a subspace $D\left[\mathcal{E}_{n}\right]$ of $C\left(X_{n}\right)$, that is, $\mathcal{F}_{n}(u)=\mathcal{E}_{n}(u, u)$ for $u \in D\left[\mathcal{E}_{n}\right]$ and $\mathcal{F}_{n}(u)=+\infty$ for $u \in C\left(X_{n}\right) \backslash D\left[\mathcal{E}_{n}\right]$, then the $\Gamma$-limit $\mathcal{F}: C(Y) \rightarrow[0, \infty)$ is also induced from a quadratic form $\mathcal{E}$ on a subspace $D[\mathcal{E}]$ of $C(Y)$. For the functional induced from a quadratic form $\mathcal{E}: D[\mathcal{E}] \times D[\mathcal{E}] \rightarrow \mathbb{R}$, we do not distinguish between the functional and the form $\mathcal{E}$. If all $\mathcal{E}_{n}$ satisfy [H-4] and/or [H-7], then so does the $\Gamma$-limit $\mathcal{E}$.

Now we consider a sequence $\left\{\left(X_{n}, R_{n}\right)\right\}$ of metric spaces associated to resistance forms $\mathcal{E}_{n}$ on sets $X_{n}$. We assume that the following conditions are satisfied:

(i) Each $\left(X_{n}, R_{n}\right)$ is compact.

(ii) There exist a compact, separable Hausdorff space $Y$, a sequence of maps $f_{n}: X_{n} \rightarrow Y$ and also a sequence of maps $h_{n}: Y \rightarrow X_{n}$ such that $f_{n} \circ h_{n}$ uniformly converges to the identity map of $Y$ as $n \rightarrow \infty$.

(iii) The functional $\mathcal{E}_{n} \Gamma$-converges (via $f_{n}$ ) to a functional $\mathcal{E}: C(Y) \rightarrow$ $[0,+\infty]$ as $n \rightarrow \infty$.

(iv) For any sequence of functions $u_{n} \in D\left[\mathcal{E}_{n}\right]$ such that $\sup _{n} \max _{X_{n}}\left|u_{n}\right|$ $<+\infty$ and $\sup _{n} \mathcal{E}_{n}\left(u_{n}\right)<+\infty$, there exists a subsequence $\left\{u_{m}\right\}$ which uniformly converges to a function $u \in C(Y)$ as $m \rightarrow \infty$.

Our main result is stated in

Theorem 5.5 Under the above conditions, the following assertions hold:

(v) Let

$$
R_{\mathcal{E}}(x, y)=\sup \left\{\frac{|u(x)-u(y)|^{2}}{\mathcal{E}(u)} \mid u \in D[\mathcal{E}], \mathcal{E}(u) \neq 0\right\}, \quad x, y \in Y .
$$

Then $R_{\mathcal{E}}: Y \times Y \rightarrow[0,+\infty]$ induces a continuous pseudo-distance on $Y$ (admitting $+\infty$ in its values), and one has

$$
0 \leq R_{\mathcal{E}}(x, y)=\lim _{n \rightarrow \infty} R_{n}\left(h_{n}(x), h_{n}(y)\right) \leq+\infty, \quad x, y \in Y .
$$


(vi) On $Y$, an equivalence relation $\sim_{b}$ is introduced as follows: $x \sim_{b} y$ if and only if $R_{\mathcal{E}}(x, y)<+\infty$. Then $Y$ is decomposed into a finite number of the equivalence classes $Y_{\alpha}(\alpha=1, \ldots, p)$; each class $Y_{\alpha}$ is open and closed in $Y$. Moreover for each $\alpha$ and large $n$, the inverse image of $Y_{\alpha}$ by $f_{n}$, $X_{n ; \alpha}=f_{n}^{-1}\left(Y_{\alpha}\right)$, is open and closed in $X_{n}$ and one has

$$
\begin{gathered}
\lim _{n \rightarrow \infty} \sup \left\{\left|R_{n}(x, y)-R_{\mathcal{E}}\left(f_{n}(x), f_{n}(y)\right)\right| \mid x, y \in X_{n ; \alpha}\right\}=0 ; \\
\lim _{n \rightarrow \infty} \sup _{y \in Y_{\alpha}} R_{\mathcal{E}}\left(f_{n}\left(X_{n ; \alpha}\right), y\right)=0 .
\end{gathered}
$$

(vii) Let $\chi_{\alpha}$ be the characteristic function of the subspace $Y_{\alpha}(1 \leq \alpha \leq p)$. Then $\chi_{\alpha} \in D[\mathcal{E}]$ and $\mathcal{E}\left(\chi_{\alpha}, u\right)=0$ for all $u \in D[\mathcal{E}]$, and if $\mathcal{E}(u, u)=0$, then $u$ is a linear combination of the characteristic functions $\chi_{\alpha}(1 \leq \alpha \leq p)$. Moreover let $D\left[\mathcal{E}_{\alpha}\right]=\left\{u \in D[\mathcal{E}] \mid \operatorname{supp} u \subset Y_{\alpha}\right\}$ and $\mathcal{E}_{\alpha}(u, v)=\mathcal{E}(u, v)$ for $u, v \in D\left[\mathcal{E}_{\alpha}\right]$. Then $D[\mathcal{E}]=\sum_{\alpha=1}^{p} D\left[\mathcal{E}_{\alpha}\right]$ and $\mathcal{E}(u, v)=\sum_{\alpha=1}^{p} \mathcal{E}_{\alpha}\left(\chi_{\alpha} u, \chi_{\alpha} v\right)$.

(viii) Another equivalence relation $\sim_{0}$ on $Y$ is introduced as follows: $x \sim_{0} y$ if and only if $R_{\mathcal{E}}(x, y)=0$. Let $Y^{*}=Y / \sim_{0}$ and $Y_{\alpha}^{*}=Y_{\alpha} / \sim_{0}$ $(\alpha=1, \ldots, p)$ be respectively the quotient spaces of $Y$ and $Y_{\alpha}$. Then for each $\alpha, R_{\mathcal{E}}$ provides $Y_{\alpha}^{*}$ a distance $R_{\alpha}$ which induces the same topology as the original one, and $D\left[\mathcal{E}_{\alpha}\right]$ is included in the pull-back of $C\left(Y_{\alpha}^{*}\right)$ by the canonical projection $\rho_{\alpha}$ of $Y_{\alpha}$ onto $Y_{\alpha}^{*}$. Thus the form $\mathcal{E}_{\alpha}$ can be assumed to be defined on $C\left(Y_{\alpha}^{*}\right) ;\left(\mathcal{E}_{\alpha}, D\left[\mathcal{E}_{\alpha}\right]\right)$ becomes a resistance form on $Y_{\alpha}^{*}$ and $R_{\alpha}$ is the associated resistance metric, that is,

$$
R_{\alpha}\left(x^{*}, y^{*}\right)=\sup \left\{\frac{\left|u\left(x^{*}\right)-u\left(y^{*}\right)\right|^{2}}{\mathcal{E}_{\alpha}(u)} \mid u \in D\left[\mathcal{E}_{\alpha}\right], \mathcal{E}_{\alpha}(u) \neq 0\right\}, \quad x^{*}, y^{*} \in Y_{\alpha}^{*} .
$$

Moreover a sequence of the compact metric spaces $\left(X_{n ; \alpha}, R_{n}\right)$ converges to $\left(Y_{\alpha}^{*}, R_{\alpha}\right)$ as $n \rightarrow \infty$ in the Gromov-Hausdorff sense via the approximating maps $\rho_{\alpha} \circ f_{n}: X_{n ; \alpha} \rightarrow Y_{\alpha}^{*}$, and the form $\mathcal{E}_{X_{n ; \alpha}}^{*}$ on $C\left(X_{n ; \alpha}\right) \Gamma$-converges to $\mathcal{E}_{\alpha}$ as $n \rightarrow \infty$.

Now we mention two consequences of the theorem.

Corollary 5.6 Let $\left\{K_{n}\right\}$ be a sequence of subspaces of compact geodesic graphs $\left|\Gamma_{n}\right|$ associated to connected, finite networks $\Gamma_{n}=\left(V_{n}, E_{n}, r_{n}\right)$, and suppose that the metric space $K_{n}$ with the induced geodesic distance $d_{r_{n}}$ converges to a compact metric space $\left(X, d_{X}\right)$ in the Gromov-Hausdorff sense via approximating maps $f_{n}: K_{n} \rightarrow X$. Then the resistance metric $R_{\left|\Gamma_{n}\right|}$ restricted to $K_{n}$ converges to a continuous pseudo-distance $R$ on $X$ with respect to the Gromov-Hausdorff distance (via the same approximating maps) 
if and only if the resistance form $\mathcal{E}_{K_{n}}^{*} \Gamma$-converges to a functional $\mathcal{E}$ on $C(X)$ as $n \rightarrow \infty$. In these cases, one has

$$
0 \leq R(x, y) \leq d_{X}(x, y), \quad x, y \in X
$$

and $R$ is given by

$$
R(x, y)=\sup \left\{\frac{|u(x)-u(y)|^{2}}{\mathcal{E}(u)} \mid u \in D[\mathcal{E}], \mathcal{E}(u) \neq 0\right\}, \quad x, y \in X .
$$

Corollary 5.7 Let $\left\{\left(X_{n}, R_{n}\right)\right\}$ be a sequence of compact metric spaces of resistance forms $\mathcal{E}_{n}$ which converges to a compact metric space $\left(Y, R_{Y}\right)$ in the Gromov-Hausdorff sense via approximating maps $f_{n}: X_{n} \rightarrow Y$. Then the resistance form $\mathcal{E}_{n} \Gamma$-converges, as $n \rightarrow \infty$, to a resistance form $\mathcal{E}$ on $C(Y)$ via the approximating maps $f_{n}$, and the limit distance $R_{Y}$ is the resistance metric associated to the form $\mathcal{E}$.

In these corollaries, the definition of a resistance metric allows us to apply Ascoli-Arzelà's theorem to the sequences and verify that the compactness condition (iv) holds true.

Proof of Theorem 5.5. The proof will be divided into 4 steps.

Step 1. We first note that for $u \in D[\mathcal{E}]$ and a sequence of $u_{n} \in D\left[\mathcal{E}_{n}\right]$ which uniformly converges to $u$ as $n \rightarrow \infty$, we have

$$
\frac{|u(x)-u(y)|^{2}}{\mathcal{E}(u)} \geq \limsup _{n \rightarrow \infty} \frac{\left|u_{n}\left(h_{n}(x)\right)-u_{n}\left(h_{n}(y)\right)\right|^{2}}{\mathcal{E}_{n}\left(u_{n}\right)}
$$

for all $x, y \in Y$, since $\mathcal{E}(u) \leq \liminf _{n \rightarrow \infty} \mathcal{E}_{n}\left(u_{n}\right)$.

Now for any $u \in D[\mathcal{E}]$, we take a sequence of $u_{n} \in D\left[\mathcal{E}_{n}\right]$ in such a way that $u_{n}$ uniformly converges to $u$ and $\mathcal{E}_{n}\left(u_{n}\right)$ tends to $\mathcal{E}(u)$ as $n \rightarrow \infty$. Then we have

$$
\begin{aligned}
\frac{|u(x)-u(y)|^{2}}{\mathcal{E}(u)} & =\lim _{n \rightarrow \infty} \frac{\left|u_{n}\left(h_{n}(x)\right)-u_{n}\left(h_{n}(y)\right)\right|^{2}}{\mathcal{E}_{n}\left(u_{n}\right)} \\
& \leq \liminf _{n \rightarrow \infty} R_{\mathcal{E}_{n}}\left(h_{n}(x), h_{n}(y)\right)(\leq+\infty)
\end{aligned}
$$

for all $x, y \in Y$. This shows that

$$
R_{\mathcal{E}}(x, y) \leq \liminf _{n \rightarrow \infty} R_{\mathcal{E}_{n}}\left(h_{n}(x), h_{n}(y)\right)(\leq+\infty), \quad x, y \in Y .
$$


Given distinct two points $x, y \in Y$, let $r=\lim \sup _{n \rightarrow \infty} R_{\mathcal{E}_{n}}\left(h_{n}(x), h_{n}(y)\right)$ $(\leq+\infty)$ and then take a subsequence $\{m\}$ such that $R_{\mathcal{E}_{m}}\left(h_{m}(x), h_{m}(y)\right)$ tends to $r$ as $m \rightarrow \infty$. For $x_{m}=h_{m}(x)$ and $y_{m}=h_{m}(y)$, let $v_{m}$ be a unique function in $D\left[\mathcal{E}_{m}\right]$ satisfying $v_{m}\left(x_{m}\right)=R_{\mathcal{E}_{m}}\left(x_{m}, y_{m}\right)^{1 / 2}, v_{m}\left(y_{m}\right)=0$ and $\mathcal{E}_{m}\left(v_{m}\right)=1$.

Suppose first that $r$ is finite. Then $\left\{v_{m}\right\}$ is uniformly bounded, since $0 \leq v_{m} \leq R_{\mathcal{E}_{m}}\left(x_{m}, y_{m}\right)^{1 / 2}$. Therefore by condition (iv), passing to a subsequence if necessarily, we may assume that $v_{m}$ uniformly converges to a function $v \in D[\mathcal{E}]$ as $m \rightarrow \infty$. Then $v(x)=r^{1 / 2}, v(y)=0$ and $\mathcal{E}(v) \leq$ $\liminf _{m \rightarrow \infty} \mathcal{E}_{m}\left(v_{m}\right)=1$. In the case where $\mathcal{E}(v)>0$, we have $R_{\mathcal{E}}(x, y) \geq$ $|v(x)-v(y)|^{2} / \mathcal{E}(v) \geq r$, and hence it follows from (5.1) that $R_{\mathcal{E}}(x, y)=r=$ $\lim _{m \rightarrow \infty} R_{\mathcal{E}_{m}}\left(x_{m}, y_{m}\right)$; in addition, $v$ satisfies $v(x)=R_{\mathcal{E}}(x, y)^{1 / 2}, v(y)=0$ and $\mathcal{E}(v)=1$. In the case where $\mathcal{E}(v)=0$, we can deduce that $v(x)=0$, and as a result, $r=0$ and hence $\lim _{m \rightarrow \infty} R_{\mathcal{E}_{m}}\left(h_{n}(x), h_{n}(y)\right)=R_{\mathcal{E}}(x, y)=0$. In fact, suppose contrarily that $v(x) \neq 0$; then for $w \in D[\mathcal{E}]$ with $\mathcal{E}(w)>0$ and $w(y)=0$, and for any $\varepsilon>0$, we would have $|v(x)+\varepsilon w(x)|^{2} / \mathcal{E}(v+\varepsilon w)=$ $|v(x)+\varepsilon w(x)|^{2} / \varepsilon^{2} \mathcal{E}(w) \leq R_{\mathcal{E}}(x, y)$, and hence letting $\varepsilon \rightarrow 0$, we would get $R_{\mathcal{E}}(x, y)=+\infty$ and hence $r=+\infty$ by (5.1). This is a contradiction.

Suppose secondly that $r=+\infty$. Let $w_{m}$ be a unique function in $D\left[\mathcal{E}_{m}\right]$ such that $w_{m}\left(x_{m}\right)=1, w_{m}\left(y_{m}\right)=0$ and $\mathcal{E}_{m}\left(w_{m}\right)=R_{\mathcal{E}_{m}}\left(x_{m}, y_{m}\right)^{-1}$. Due to the maximum principle, we see that $0 \leq w_{m} \leq 1$, and the assumption implies that $\mathcal{E}_{m}\left(w_{m}\right)$ tends to 0 as $m \rightarrow \infty$. Therefore passing to a subsequence, we may assume that $w_{m}$ uniformly converges to a function $w \in D[\mathcal{E}]$ such that $w(x)=1, w(y)=0$ and $\mathcal{E}(w)=0$. This implies that $R_{\mathcal{E}}(x, y)=\lim _{m \rightarrow \infty} R_{\mathcal{E}_{m}}\left(h_{m}(x), h_{m}(y)\right)=+\infty$. Thus we have shown that $0 \leq R_{\mathcal{E}}(x, y)=\lim _{m \rightarrow \infty}\left(h_{m}(x), h_{m}(y)\right) \leq+\infty$.

Now we have two equivalence relations $\sim_{0}$ and $\sim_{b}$ on $Y$ defined respectively by $x \sim_{0} y \Leftrightarrow R_{\mathcal{E}}(x, y)=0$ and $x \sim_{b} y \Leftrightarrow R_{\mathcal{E}}(x, y)<+\infty$. We denote by $Y^{*}$ and $Y^{* *}$ respectively the quotient spaces induced from the equivalence relations $\sim_{0}$ and $\sim_{b}$. Then we are allowed to assume that $D[\mathcal{E}]$ is a subalgebra of the space of continuous functions on $Y^{*}, C\left(Y^{*}\right)$, and $\mathcal{E}$ is a functional on $C\left(Y^{*}\right)$ with values in $[0,+\infty]$.

Step 2. In this step, we prove that $R_{\mathcal{E}}: Y \times Y \rightarrow[0,+\infty]$ is continuous. We first claim that $R_{\mathcal{E}_{n(i)}}\left(h_{n(i)}\left(x_{i}\right), h_{n(i)}(y)\right)$ goes to zero as $i \rightarrow \infty$ for any $y \in Y$ and a sequence of points $x_{i} \in Y$ tending to $y$, and for every divergent sequence $\{n(i)\}$.

To see this, suppose contrarily that $\lim \sup _{i \rightarrow \infty} R_{\mathcal{E}_{n(i)}}\left(h_{n(i)}\left(x_{i}\right), h_{n(i)}(y)\right)$ $=+\infty$. Then we choose a subsequence, say $\{n(j)\}$, in such a way that $R_{\mathcal{E}_{n(j)}}\left(h_{n(j)}\left(x_{j}\right), h_{n(j)}(y)\right)$ diverges to infinity as $j \rightarrow \infty$. Let $v_{n(j)}$ be a unique function of $D\left[\mathcal{E}_{n(j)}\right]$ such that $v_{n(j)}\left(h_{n(j)}\left(x_{j}\right)\right)=1, v_{n(j)}\left(h_{n(j)}(y)\right)=0$ and $\mathcal{E}_{n(j)}\left(v_{n(j)}\right)=R_{\mathcal{E}_{n(j)}}\left(h_{n(j)}\left(x_{j}\right), h_{n(j)}(y)\right)^{-1}$. Passing to a subsequence, 
we may assume that $v_{n(j)}$ uniformly converges to a function $v \in D[\mathcal{E}]$ with $\mathcal{E}(v)=0$. Since $\lim _{j \rightarrow \infty}\left|v\left(f_{n(j)} \circ h_{n(j)}\left(x_{j}\right)\right)-1\right|=\lim _{j \rightarrow \infty} \mid v\left(f_{n(j)} \circ\right.$ $\left.h_{n(j)}\left(x_{j}\right)\right)-v_{n(j)}\left(h_{n(j)}\left(x_{j}\right)\right) \mid=0, \lim _{j \rightarrow \infty} f_{n(j)} \circ h_{n(j)}\left(x_{j}\right)=y$ and $v$ is continuous, we get $v(y)=1$. On the other hand, since $\lim _{j \rightarrow \infty}\left|v\left(f_{n(j)} \circ h_{n(j)}(y)\right)\right|=$ $\lim _{j \rightarrow \infty}\left|v\left(f_{n(j)} \circ h_{n(j)}(y)\right)-v_{n(j)}\left(h_{n(j)}(y)\right)\right|=0$, we get $v(y)=0$. This is a contradiction. Thus $R_{\mathcal{E}_{n(i)}}\left(h_{n(i)}\left(x_{i}\right), h_{n(i)}(y)\right)$ is bounded as $i \rightarrow \infty$.

Let $w_{n(i)}$ be a unique function of $D\left[\mathcal{E}_{n(i)}\right]$ satisfying $w_{n(i)}\left(h_{n(i)}\left(x_{i}\right)\right)=$ $R_{\mathcal{E}_{n(i)}}\left(h_{n(i)}\left(x_{i}\right), h_{n(i)}(y)\right)^{1 / 2}, w_{n(i)}\left(h_{n(i)}(y)\right)=0$ and $\mathcal{E}_{n(i)}\left(w_{n(i)}\right)=1$. Passing to a subsequence, we assume that $w_{n(i)}$ uniformly converges to a function $w \in D[\mathcal{E}]$ as $i \rightarrow \infty$. Then $\left|w_{n(i)}\left(h_{n(i)}\left(x_{i}\right)\right)-w\left(f_{n(i)} \circ h_{n(i)}\left(x_{i}\right)\right)\right|$ tends to zero as $i \rightarrow \infty$ and hence we have $w(y)=\lim _{i \rightarrow \infty} w\left(f_{n(i)} \circ h_{n(i)}\left(x_{i}\right)\right)=$ $\lim _{i \rightarrow \infty} R_{\mathcal{E}_{n(i)}}\left(h_{n(i)}\left(x_{i}\right), h_{n(i)}(y)\right)^{1 / 2}$. On the other hand, we have $w(y)=$ $\lim _{i \rightarrow \infty} w\left(f_{n(i)} \circ h_{n(i)}(y)\right)=0$, since $\left|w\left(f_{n(i)} \circ h_{n(i)}(y)\right)\right|=\mid w\left(f_{n(i)} \circ h_{n(i)}(y)\right)-$ $w_{n(i)}\left(h_{n(i)}(y)\right) \mid$ tends to zero as $i \rightarrow \infty$; thus $\lim _{i \rightarrow \infty} R_{\mathcal{E}_{n(i)}}\left(h_{n(i)}\left(x_{i}\right), h_{n(i)}(y)\right)$ $=0$. As a result, we see that $R_{\mathcal{E}}(x, y)$ goes to zero as $x \rightarrow y$ and hence $R_{\mathcal{E}}: Y \times Y \rightarrow[0,+\infty]$ is continuous. Note also that each equivalence class with respect to the relation $\sim_{b}$ is open and closed. Since $Y$ is compact, $Y^{* *}$ is a finite set, and $Y$ is decomposed into the $p$ equivalence classes $Y_{1}, \ldots, Y_{p}$. Notice that $R_{\mathcal{E}}$ is bounded if $Y$ is connected.

Step 3. In this step, we show that

(i) $\lim _{n \rightarrow \infty} \sup _{x \in X_{n}} R_{\mathcal{E}_{n}}\left(x, h_{n} \circ f_{n}(x)\right)=0$ and

(ii) $\lim _{n \rightarrow \infty} \sup _{x, y \in X_{n ; \alpha}}\left|R_{\mathcal{E}_{n}}(x, y)-R_{\mathcal{E}}\left(f_{n}(x), f_{n}(y)\right)\right|=0 \quad$ for each $\alpha=1, \ldots, p$.

To prove the first assertion (i), let us consider a sequence of points $x_{n} \in X_{n}$ and let $y_{n}=h_{n} \circ f_{n}\left(x_{n}\right) \in X_{n}$. For the proof, we may assume that $x_{n} \neq y_{n}$. Let $v_{n}$ be a unique function of $D\left[\mathcal{E}_{n}\right]$ satisfying $v_{n}\left(x_{n}\right)=R_{\mathcal{E}_{n}}\left(x_{n}, y_{n}\right)^{1 / 2}$, $v_{n}\left(y_{n}\right)=0$ and $\mathcal{E}_{n}\left(v_{n}\right)=1$. In the case where $R_{\mathcal{E}_{n}}\left(x_{n}, y_{n}\right)$ is bounded as $n \rightarrow \infty$, passing to a subsequence, we may assume that $v_{n}$ uniformly converges to a function $v \in D[\mathcal{E}]$ as $n \rightarrow \infty$. Then $\left|v_{n}\left(x_{n}\right)-v\left(f_{n}\left(x_{n}\right)\right)\right|$, $\left|v_{n}\left(y_{n}\right)-v\left(f_{n}\left(y_{n}\right)\right)\right|$ and $d\left(f_{n}\left(x_{n}\right), f_{n}\left(y_{n}\right)\right)$ tend to zero as $n \rightarrow \infty$, where $d$ is a distance on $Y$ that induces the same topology of $Y$, and hence we get $\lim _{n \rightarrow \infty} R_{\mathcal{E}_{n}}\left(x_{n}, y_{n}\right)=\lim _{n \rightarrow \infty} v_{n}\left(x_{n}\right)^{2}=\lim _{n \rightarrow \infty} v_{n}\left(y_{n}\right)^{2}=0$. This shows the first assertion, since $\left\{x_{n}\right\}$ is arbitrarily chosen. In the case where $R_{\mathcal{E}_{n}}\left(x_{n}, y_{n}\right)$ diverges to infinity as $n \rightarrow \infty$, we consider a sequence of functions $w_{n}=v_{n} / R_{\mathcal{E}_{n}}\left(x_{n}, y_{n}\right)^{1 / 2}$. Then passing to a subsequence, we may assume that $w_{n}$ uniformly converges to a function $w \in D[\mathcal{E}]$ as $n \rightarrow \infty$ and we can deduce that $\lim _{n \rightarrow \infty} w\left(f_{n}\left(x_{n}\right)\right)=1$ and $\lim _{n \rightarrow \infty} w\left(f_{n}\left(y_{n}\right)\right)=0$. But this contradicts to the continuity of $w$, since $d\left(f_{n}\left(x_{n}\right), f_{n}\left(y_{n}\right)\right)$ tends to zero as $n \rightarrow \infty$. 
Step 4. Given a closed subset $K$ of $Y$ and a continuous function $u$ on $K$, we set as before $\mathcal{A}_{u}:=\left\{v \in D[\mathcal{E}] \mid v_{\mid K}=u\right\}, D\left[\mathcal{E}_{K}^{*}\right]:=\left\{u \in C(K) \mid \mathcal{A}_{u} \neq \emptyset\right\}$, and $\mathcal{E}_{K}^{*}(u):=\inf \left\{\mathcal{E}(v) \mid v \in \mathcal{A}_{u}\right\}$. When $\mathcal{A}_{u}$ is empty, we understand $\mathcal{E}_{K}^{*}(u)=+\infty$, and when $\mathcal{A}_{u}$ is not empty, there exist minimizers $h$ in $\mathcal{A}_{u}$. They are uniquely determined on $Y_{\alpha}$ if $K \cap Y_{\alpha} \neq \emptyset$, and they are constants, not uniquely determined, on $Y_{\alpha}$ otherwise. In what follows, we consider a finite subset $K=\left\{x_{1}, \ldots, x_{N}\right\}$ of $Y$ such that $0<R_{\mathcal{E}}\left(x_{i}, x_{j}\right)(\leq+\infty)$ for any pair of $i, j$ with $i \neq j$, and further $K$ intersects every $Y_{\alpha}$. For any $u \in \ell(K)$, we denote by $H_{K ; u}$ the unique minimizer of $\mathcal{A}_{u}$. Let $K_{n}=$ $\left\{h_{n}\left(x_{1}\right), \ldots, h_{n}\left(x_{N}\right)\right\} \subset X_{n}$. Since $R_{\mathcal{E}_{n}}\left(h_{n}\left(x_{i}\right), h_{n}\left(x_{j}\right)\right)$ tends to $R_{\mathcal{E}}\left(x_{i}, x_{j}\right)$ as $n \rightarrow \infty, h_{\mid K}: K \rightarrow K_{n}$ is bijective and the trace $\mathcal{E}_{K_{n}}^{*}$ on $K_{n} \Gamma$-converges to the trace $\mathcal{E}_{K}^{*}$ on $K$ as $n \rightarrow \infty$. Let $\chi_{i}=\chi_{x_{i}} \in \ell(K)$ and $\chi_{n ; i}=\chi_{h_{n}\left(x_{i}\right)} \in$ $\ell\left(K_{n}\right)$ for simplicity. Then we can deduce that $\mathcal{E}_{K_{n}}^{*}\left(\chi_{n ; i}, \chi_{n ; i}\right)$ is bounded as $n \rightarrow \infty$. In fact, letting $c_{n ; i, j}=-\mathcal{E}_{K_{n}}^{*}\left(\chi_{n ; i}, \chi_{n ; j}\right)$ and recalling (1.5), we have

$$
\sum_{i, j=1}^{N} c_{n ; i, j} R_{\mathcal{E}_{n}}\left(h_{n}\left(x_{i}\right), h_{n}\left(x_{j}\right)\right)=2(N-1),
$$

and hence

$$
0 \leq c_{n ; i, j} \leq 2(N-1) R_{\mathcal{E}_{n}}\left(h_{n}\left(x_{i}\right), h_{n}\left(x_{j}\right)\right)^{-1}
$$

for $i \neq j$. The right side tends to $(N-1) R_{\mathcal{E}}\left(x_{i}, x_{j}\right)^{-1}$ as $n \rightarrow \infty$, and hence $c_{n ; i, j}$ are bounded uniformly from above by a constant $b$. Therefore we have

$$
-c_{n ; i, i}=\sum_{j \neq i} c_{n ; i, j} \leq(N-1) b .
$$

Note that as $n \rightarrow \infty, c_{n ; i, j}$ goes to zero if $x_{i} \in Y_{\alpha}$ and $x_{j} \in Y_{\beta}$ with $\alpha \neq \beta$. Moreover we claim that $\mathcal{E}_{K}^{*}\left(\chi_{i}, \chi_{j}\right)=0$ for such a pair of $i, j$. In fact, we take a function $\phi_{n ; i} \in C\left(K_{n}\right)$ in such a way that $\phi_{n ; i}$ uniformly (pointwise in this case) converges to $\chi_{i}$ and $\mathcal{E}_{K_{n}}^{*}\left(\phi_{n ; i}\right)$ tends to $\mathcal{E}_{K}^{*}\left(\chi_{i}\right)$ as $n \rightarrow \infty$. Then we see that $\mathcal{E}_{K_{n}}^{*}\left(\phi_{n ; i}, \chi_{n ; j}\right)$ goes to $\mathcal{E}_{K}^{*}\left(\chi_{i}, \chi_{j}\right)$ as $n \rightarrow \infty$. On the other hand, we have

$$
\begin{aligned}
\mathcal{E}_{K_{n}}^{*}\left(\phi_{n ; i}, \chi_{n ; j}\right)= & \sum_{k \neq j}-c_{n ; j k}\left(\phi_{n ; i}\left(h_{n}\left(x_{j}\right)\right)-\phi_{n ; i}\left(h_{n}\left(x_{k}\right)\right)\right. \\
& +\sum_{k \neq j} c_{n ; j k}\left(\phi_{n ; i}\left(h_{n}\left(x_{k}\right)\right)-\phi_{n ; i}\left(h_{n}\left(x_{j}\right)\right)\right) ;
\end{aligned}
$$

the right side tends to 0 as $n \rightarrow \infty$. Thus the claim is verified.

Now we choose an increasing sequence of finite subsets $K_{t}=\left\{x_{1}, \ldots, x_{N_{t}}\right\}$ of $Y$ in such a way that $\cup_{t} K_{t}$ is dense in $Y^{*}$ and furthermore for $x, y \in K_{t}$, $R_{\mathcal{E}}(x, y)>0$ if $x \neq y$. Then we can deduce that $u \in D[\mathcal{E}] \subset C(Y)$ if and only if $\lim _{t \rightarrow \infty} \mathcal{E}_{K_{t}}^{*}\left(u_{\mid K_{t}}\right)<+\infty$ and in this case, $\mathcal{E}(u)=\lim _{t \rightarrow \infty} \mathcal{E}_{K_{t}}^{*}\left(u_{\mid K_{t}}\right)<+\infty$. 
Moreover as we have shown, it holds that

$$
\mathcal{E}_{K_{t}}^{*}\left(u_{\mid K_{t}}, v_{\mid K_{t}}\right)=\sum_{\alpha} \mathcal{E}_{K_{t}}^{*}\left(\chi_{\alpha} u_{\mid K_{t}}, \chi_{\alpha} v_{\mid K_{t}}\right)
$$

for all $u, v \in D[\mathcal{E}]$. This proves the assertion (vii). The last (viii) follows from the others. This completes the proof of Theorem 5.5.

In the case where a sequence of connected, finite networks $\left(V, E_{n}, r_{n}\right)$ with the same set of vertices $V$ is considered, letting $f_{n}$ as in Theorem 5.5 be the identity map of $V$, we see that the condition (iv) is always satisfied. Therefore we can apply our theorem to this case. See Example 5.4, and also Colin de Verdière, Pan and Ycart [14] for related results.

5.3 Now we consider a family of resistance forms $\mathcal{E}$ on sets $X$ satisfying [H-6] endowed with Borel measures $\mu$ on $X$ such that $\mu(X)=1$ and the resistance metrics $R_{\mathcal{E}}$ are bounded from above by a positive constant $D^{2}$. Then as shown in subsection 3.2, we have isometric embeddings of the metric spaces $\left(X, \mathcal{S}_{\mu}\right)$ into a fixed compact subset $B^{1,2}(r(D))$ of the Hilbert space $\ell^{2}$. Recall here the fact that the set of closed subspaces of a compact metric space is indeed compact with respect to the Hausdorff distance (cf. e.g., [9]). We intend to apply this fact to our family.

Let $\left(X_{n}, \mathcal{E}_{n}, \mu_{n}\right)$ be a sequence in the family. Suppose for simplicity that the measure $\mu_{n}$ is positive on each $X_{n}$, that is $\operatorname{supp} \mu_{n}=X_{n}$. Let $\left\{\lambda_{n ; i} \mid i=\right.$ $0,1, \ldots\}$ be the set of eigenvalues in nondecreasing order of the self-adjoint operator $\mathcal{L}_{n}$ associated to the form $\mathcal{E}_{n}$ on $L^{2}\left(X_{n}, \mu_{n}\right)$. We take a complete orthonormal system $\Phi_{n}$ of eigenfunctions $\phi_{n ; i}$ of $\mathcal{L}_{n}$ with the $i$-th eigenvalues $\lambda_{n ; i}$, and consider the map $J_{n}: X_{n} \rightarrow \ell^{2}$ defined by $J_{n}(x)=\left(e^{-\lambda_{n ; i} / 2} \phi_{n ; i}\right)$ as in subsection 3.2. Then every image $J_{n}\left(X_{n}\right)$ of $X_{n}$ stays in a compact subset $B^{1,2}(r(D))$ of $\ell^{2}$. Therefore passing to a subsequence, we assume that $J_{n}\left(X_{n}\right)$ converges to a compact subspace $Z$ of $B^{1,2}(r(D))$ with respect to the Hausdorff distance on the set of closed subsets of $B^{1,2}(r(D))$. In other words, there exists a sequence of positive numbers $\varepsilon_{n}$ tending to zero as $n \rightarrow \infty$ such that for any point $x \in X_{n}$, we find a point $f_{n}(x) \in Z$ satisfying $\left\|f_{n}(x)-J_{n}(x)\right\|_{\ell^{2}} \leq \varepsilon_{n}$, and also for any point $a \in Z$, we get a point $h_{n}(a) \in X_{n}$ satisfying $\left\|a-J_{n}\left(h_{n}(a)\right)\right\|_{\ell^{2}} \leq \varepsilon_{n}$. Using the coordinate functions $\gamma_{i}: \ell^{2} \rightarrow \mathbb{R}(i=1,2, \ldots)$ of $\ell^{2}, \gamma_{i}\left(\left(x_{j}\right)\right)=x_{i}$, these inequalities are written as follows: $\sum_{i=1}^{\infty}\left(\gamma_{i}\left(f_{n}(x)\right)-e^{-\lambda_{n ; i} / 2} \phi_{n ; i}(x)\right)^{2} \leq \varepsilon_{n}^{2}$, for all $x \in X_{n}$; $\sum_{i=1}^{\infty}\left(\gamma_{i}(a)-e^{-\lambda_{n ; i} / 2} \phi_{n ; i}\left(h_{n}(a)\right)\right)^{2} \leq \varepsilon_{n}^{2}$ for all $a \in Z$.

Now using the eigenvalue estimate in (3.6) and passing to a subsequence, we assume that for each $i, \lambda_{n ; i}$ goes to an extended number $\lambda_{i} \in(0,+\infty]$ as $n \rightarrow \infty$. We define functions $\phi_{i}$ on $Z$ by

$$
\phi_{i}(a)=e^{\lambda_{i} / 2} \gamma_{i}(a), \quad a \in Z .
$$


In the case where $\lambda_{N-1}<+\infty$ but $\lambda_{N}=+\infty$ for some finite $N$, we have $\gamma_{i}(a)=0$ for all $i \geq N$, and we assume that $\phi_{i}=0$ for all $i \geq N$ for our convenience. Then the inequalities mentioned above are expressed as

$$
\begin{gathered}
\sum_{i=1}^{\infty}\left(e^{-\lambda_{i} / 2} \phi_{i}\left(f_{n}(x)\right)-e^{-\lambda_{n ; i} / 2} \phi_{n ; i}(x)\right)^{2} \leq \varepsilon_{n}^{2}, \quad x \in X_{n} \\
\sum_{i=1}^{\infty}\left(e^{-\lambda_{i} / 2} \phi_{i}(a)-e^{-\lambda_{n ; i} / 2} \phi_{n ; i}\left(h_{n}(a)\right)^{2} \leq \varepsilon_{n}^{2}, \quad a \in Z .\right.
\end{gathered}
$$

In particular, for each $i, \phi_{n ; i}$ uniformly converges to $\phi_{i}$ as $n \rightarrow \infty$.

In view of Theorem 5.4, we can pass to a subsequence so that the functional $\mathcal{E}_{n}$ on $C\left(X_{n}, S^{\mu_{n}}\right) \Gamma$-converges to a functional $\mathcal{E}$ on $C(Z)$ as $n \rightarrow \infty$. Then we observe that $\phi_{i} \in D[\mathcal{E}]$ and $\mathcal{E}\left(\phi_{i}\right) \leq \lambda_{i}$, since

$$
\mathcal{E}\left(\phi_{i}\right) \leq \liminf _{n \rightarrow \infty} \mathcal{E}_{n}\left(\phi_{n ; i}\right)=\liminf _{n \rightarrow \infty} \lambda_{n ; i}=\lambda_{i} .
$$

Let

$$
R_{\mathcal{E}}(a, b)=\sup \left\{\frac{|u(a)-u(b)|^{2}}{\mathcal{E}(u)} \mid u \in D[\mathcal{E}], \mathcal{E}(u)>0\right\}, \quad a, b \in Z .
$$

Then $0<R_{\mathcal{E}}(a, b) \leq D^{2}$ for all $a, b \in Z$ with $a \neq b$, since we have $\mid u(a)-$ $\left.u(b)\right|^{2} \leq D^{2} \mathcal{E}(u)$ for all $u \in D[\mathcal{E}]$; the positivity of $R_{\mathcal{E}}$ is a consequence of the separation of points of $Z$ by the family $\left\{\phi_{i}\right\}$. The functional $\mathcal{E}$ satisfies [H-2'], namely it is lower semi-continuous with respect to the uniform norm of $C(Z)$, hence [H-3] follows. Obviously [H-4] and [H-6] hold true.

Since $\mu_{n}\left(X_{n}\right)=1$, by passing to a subsequence, we assume that the image measure $f_{n *} \mu_{n}$ vaguely converges to a Radon measure $\mu$ on $Z$ as $n \rightarrow \infty$. Let $K$ be the support of the limit measure $\mu$ and consider the trace $\mathcal{E}_{K}^{*}$ on $K$ of $\mathcal{E}$. Let $I$ be a nonnegative integer and $u$ a function in $D\left[\mathcal{E}_{K}^{*}\right]$ such that $\int u \psi_{i} d \mu=0$ for $i=0,1, \ldots, I-1$, where we put $\psi_{i}=\phi_{i \mid K}$ and no conditions are imposed if $I=0$. Let $H_{K ; u}$ be the unique minimizer in $\mathcal{A}_{u}$, and then choose a sequence of functions $u_{n} \in D\left[\mathcal{E}_{n}\right]$ such that as $n \rightarrow \infty$, $u_{n}$ uniformly converges to $H_{K ; u}$ and $\mathcal{E}_{n}\left(u_{n}\right)$ tends to $\mathcal{E}_{K}^{*}(u)=\mathcal{E}\left(H_{K ; u}\right)$. Let $u_{n ; I}=u_{n}-\sum_{i=0}^{I-1}\left(\int u_{n} \phi_{n ; i} d \mu_{n}\right) \phi_{n ; i}$, where we understand $u_{n ; 0}=u_{n}$ if $I=0$. Then we have

$$
\begin{aligned}
& \lambda_{n ; I} \int u_{n ; I}^{2} d \mu_{n} \leq \mathcal{E}_{n}\left(u_{n ; I}\right) \\
& \quad=\mathcal{E}_{n}\left(u_{n}\right)-2 \sum_{i=0}^{I-1} \int u_{n} \phi_{n ; i} d \mu_{n} \mathcal{E}_{n}\left(u_{n}, \phi_{n ; i}\right)+\sum_{i=0}^{I-1} \lambda_{n ; i}\left(\int u_{n} \phi_{n ; i} d \mu_{n}\right)^{2} \\
& \quad \leq \mathcal{E}_{n}\left(u_{n}\right)+2 \sum_{i=0}^{I-1} \lambda_{n ; i}^{1 / 2}\left|\int u_{n} \phi_{n ; i} d \mu_{n}\right| \mathcal{E}_{n}\left(u_{n}\right)^{1 / 2}+\sum_{i=0}^{I-1} \lambda_{n ; i}\left(\int u_{n} \phi_{n ; i} d \mu_{n}\right)^{2} .
\end{aligned}
$$


Therefore letting $n \rightarrow \infty$, we get

$$
\lambda_{I} \int u^{2} d \mu \leq \mathcal{E}_{K}^{*}(u)
$$

This shows in particular that $u=0$ if $u \in D[\mathcal{E}]$ and $\int u \psi_{i} d \mu=0$ for all $i$. Moreover applying this to $u=\psi_{i}$ and using (5.2), we have

$$
\begin{aligned}
\lambda_{I} & =\mathcal{E}_{K}^{*}\left(\psi_{I}\right)=\mathcal{E}\left(\phi_{I}\right) \\
& =\inf \left\{\mathcal{E}_{K}^{*}(u) \mid \int u^{2} d \mu=1, \int u \psi_{i} d \mu=0(i=0,1, \ldots, I-1)\right\}
\end{aligned}
$$

in addition it is easy to see that $\mathcal{E}\left(\phi_{i}, \phi_{j}\right)=\delta_{i j} \lambda_{i}$ for $i, j=0,1,2, \ldots$

Now we are given $u \in D\left[\mathcal{E}_{K}^{*}\right]$. Let $v$ be a function in $\mathcal{A}_{u}$ and $\left\{v_{n}\right\}$ a sequence of functions in $D\left[\mathcal{E}_{n}\right]$ such that $v_{n}$ uniformly converges to $v$ and $\mathcal{E}_{n}\left(v_{n}\right)$ tends to $\mathcal{E}(v)$ as $n \rightarrow \infty$. Since

$$
\sum_{i=0}^{I-1} \lambda_{i}\left(\int u \psi_{i} d \mu\right)^{2}=\lim _{n \rightarrow \infty} \sum_{i=0}^{I-1} \lambda_{n ; i}\left(\int v_{n} \phi_{n ; i} d \mu_{n}\right)^{2} \leq \lim _{n \rightarrow \infty} \mathcal{E}_{n}\left(v_{n}\right)=\mathcal{E}(v)
$$

for all positive integers $I$, we get

$$
\sum_{i=0}^{\infty} \lambda_{i}\left(\int u \psi_{i} d \mu\right)^{2} \leq \mathcal{E}(v)
$$

Let $h_{I J}=\sum_{i=I}^{J} \int u \psi_{i} d \mu \phi_{i}$ for $0<I<J<+\infty$. Then we take a point $y \in K$ so that $h_{I J}(y)=0$ (such a point exists, since $\int h_{I J} d \mu=0$ ). Then we have

$$
h_{I J}(x)^{2} \leq D^{2} \mathcal{E}\left(h_{I J}\right)=D^{2} \sum_{i=I}^{J} \lambda_{i}\left(\int u \psi_{i} d \mu\right)^{2}, \quad x \in Z .
$$

Since the right side tends to 0 as $I \rightarrow \infty, \sum_{i=0}^{\infty} \int u \psi_{i} d \mu \phi_{i}$ uniformly converges and we put $h=\sum_{i=0}^{\infty} \int u \psi_{i} d \mu \phi_{i}$. Then we obtain

$$
\mathcal{E}(h) \leq \sum_{i=0}^{\infty} \lambda_{i}\left(\int u \psi_{i} d \mu\right)^{2}
$$

This together with (5.3) implies that

$$
\mathcal{E}_{K}^{*}(u)=\mathcal{E}(h)=\sum_{i=0}^{\infty} \lambda_{i}\left(\int u \psi_{i} d \mu\right)^{2} .
$$


Thus we see that given $u \in D\left[\mathcal{E}_{K}^{*}\right], H_{K ; u}=\sum_{i=1}^{\infty} \int u \phi_{i} d \mu \phi_{i}$ in $C(Z)$ and

$$
\mathcal{E}_{K}^{*}(u)=\mathcal{E}\left(H_{K ; u_{\mid K}}\right)=\sum_{i=1}^{\infty} \lambda_{i}\left(\int u \phi_{i} d \mu\right)^{2} .
$$

In what follows, given $u \in D\left[\mathcal{E}_{K}^{*}\right]$, we write $\tilde{u}$ instead of $H_{K ; u}$ for short. To state a result on spectral convergence, we need some definitions. We define a linear map $T_{n}: D\left[\mathcal{E}_{K}^{*}\right] \rightarrow L^{2}\left(X_{n}, \mu_{n}\right)$ by $T_{n}(u)=f_{n}^{*} \tilde{u}$ for $u \in$ $D\left[\mathcal{E}_{K}^{*}\right]$. A sequence of functions $u_{n} \in L^{2}\left(X_{n}, \mu_{n}\right)$ is said to $L^{2}$ strongly (resp. $L^{2}$ weakly) converge to a function $u \in L^{2}(K, \mu)$ if there exists a sequence of functions $v_{i}$ in $D\left[\mathcal{E}_{K}^{*}\right]$ such that $\lim _{i \rightarrow \infty}\left\|v_{i}-u\right\|_{L^{2}(K, \mu)}=0$ and $\lim _{i \rightarrow \infty} \lim \sup _{n \rightarrow \infty}\left\|T_{n}\left(v_{i}\right)-u_{n}\right\|_{L^{2}\left(X_{n}, \mu_{n}\right)}=0$ (resp. if, for every $v \in$ $L^{2}(K, \mu)$ and any sequence of $v_{n} \in L^{2}\left(X_{n}, \mu_{n}\right)$ which $L^{2}$-strongly converges to $\left.v, \lim _{n \rightarrow \infty}\left(u_{n}, v_{n}\right)_{L^{2}\left(X_{n}, \mu_{n}\right)}=(u, v)_{L^{2}(K, \mu)}\right)$ (cf. [33]).

Theorem 5.8 Let $\left\{\left(X_{n}, \mathcal{E}_{n}, \mu_{n}\right)\right\}$ be a sequence of triplets $\left\{\left(X_{n}, \mathcal{E}_{n}, \mu_{n}\right)\right\}$ as above such that the effective resistance $R_{\mathcal{E}_{n}}$ is uniformly bounded from above by a positive constant $D^{2}$ and the total mass $\mu_{n}\left(X_{n}\right)$ is equal to one. Then there exist a subsequence $\left\{\left(X_{m}, \mathcal{E}_{m}, \mu_{m}\right)\right\}$ and a triplet $(Z, \mathcal{E}, \mu)$ satisfying the same conditions as above and the following:

(i) The metric space $\left(Z, S^{\mu}\right)$ is compact and the sequence of metric spaces $\left(X_{m}, S^{\mu_{m}}\right)$ converges to it in the Gromov-Hausdorff sense via approximating Borel maps $f_{m}: X_{m} \rightarrow Z$ and $h_{m}: Z \rightarrow X_{m}$.

(ii) The sequence of the forms $\mathcal{E}_{m} \Gamma$-converges to $\mathcal{E}$ and $D[\mathcal{E}] \subset C\left(Z, \tilde{S}^{\mu}\right)$.

(iii) The image measure $f_{m *} \mu_{m}$ weakly converges to $\mu$.

(iv) It holds that

$$
\tilde{S}^{\mu}(x, y) \leq R_{\mathcal{E}}(x, y)^{1 / 2} \leq \liminf _{m \rightarrow \infty} R_{m}\left(h_{m}(x), h_{m}(y)\right)^{1 / 2} \leq D, \quad x, y \in Z .
$$

(v) If a sequence of functions $u_{n} \in L^{2}\left(X_{n}, \mu_{n}\right) L^{2}$-strongly converges to a function $u \in L^{2}(K, \mu)$ as $n \rightarrow \infty$, then one has

$$
\mathcal{E}(u) \leq \liminf _{n \rightarrow \infty} \mathcal{E}_{n}\left(u_{n}\right) \leq+\infty .
$$

(vi) Let $\left\{u_{n}\right\}$ be a sequence of functions $u_{n} \in D\left[\mathcal{E}_{n}\right]$ with $\sup _{n} \mathcal{E}_{n}\left(u_{n}\right)+$ $\left\|u_{n}\right\|_{L^{2}\left(X_{n}, \mu_{n}\right)}^{2}<+\infty$. Then there exist a subsequence $\left\{u_{m}\right\}$ that $L^{2}$-strongly converges to a function $u \in D[\mathcal{E}]$ as $n \rightarrow \infty$.

(vii) Let $\left\{u_{n}\right\}$ be a sequence of functions $u_{n} \in L^{2}\left(X_{n}, \mu_{n}\right)$ such that $\sup _{n}\left\|u_{n}\right\|_{L^{2}\left(X_{n}, \mu_{n}\right)}<+\infty$ and suppose $\left\{u_{n}\right\}$ is $L^{2}$-weakly converges to a function $u \in L^{2}(K, \mu)$. Then for any $\alpha>0$, $w_{n}=\mathcal{R}_{\mu_{n} ; \alpha} u_{n}$ uniformly converges to $w=\left(\mathcal{R}_{\mu ; \alpha} u\right)^{\sim}$ and $\mathcal{E}_{n}\left(w_{n}\right)$ tends to $\mathcal{E}_{K}^{*}(w)$ as $n \rightarrow \infty$, where $\mathcal{R}_{\mu_{n} ; \alpha}$ and $\mathcal{R}_{\mu ; \alpha}$ are respectively the resolvents of the operators $\mathcal{L}_{\mu_{n}}$ and $\mathcal{L}_{\mu}$. 
Now we investigate a nonnegative quadratic form $\mathcal{E}$ on a set $X$ satisfying [H-5], [H-2] and [H-6], and endowed with finite Borel measure $\mu$ such that $\int M_{\mathcal{E}}(x) d \mu(x)<+\infty$. Let $K=\operatorname{supp} \mu$ and $\Phi=\left\{\phi_{i} \mid i=1,2, \ldots\right\}$ a complete orthonormal system of eigenfunctions $\phi_{i}$ of the operator $\mathcal{L}_{\mu}$ acting on $L^{2}(K, \mu)$ with eigenvalues $\lambda_{i}$. The Green function $g_{\mathcal{E}}(x, y)$ is expressed on $K$ as

$$
g_{\mathcal{E}}(x, y)=\sum_{i=1}^{\infty} \frac{1}{\lambda_{i}} \phi_{i}(x) \phi_{i}(y), \quad x, y \in K .
$$

In particular, we have

$$
\int g_{\mathcal{E}}(x, x) d \mu(x)=\sum_{i=1}^{\infty} \frac{1}{\lambda_{i}}
$$

so that

$$
\lambda_{i} \geq \frac{i}{\int g_{\mathcal{E}}(x, x) d \mu(x)}, \quad i=1,2, \ldots
$$

Let $S^{\mu}(x, y)=\left(\sum_{i=1}^{\infty} e^{-\lambda_{i}}\left(\phi_{i}(x)-\phi_{i}(y)\right)^{2}\right)^{1 / 2}$ for $x, y \in K$. Then $S^{\mu}$ provides a distance on $K$ which is isometrically realized in $\ell^{2}$ by an imbedding $J_{\Phi}$ : $K \rightarrow \ell$ defined by $J_{\Phi}(x)=\left(e^{-\lambda_{i} / 2} \phi_{i}(x)\right), x \in K$.

Suppose that $\sup _{x \in K} g_{\mathcal{E}}(x, x) \leq D^{2}$ and $\mu(K) \leq M$ for some positive numbers $D$ and $M$. Then the image $J_{\Phi}(K)$ is included in a compact space in $\ell^{2}$, so that the completion $\left(\bar{K}, S^{\mu}\right)$ is compact. Moreover as in Lemma 3.8, every function $u \in D[\mathcal{E}]$ extends to a continuous function $\bar{u}$ on $\bar{K}$ and the eigenfunction expansion of $\bar{u}, \bar{u}=\sum_{i=1}^{\infty} \int u \phi_{i} d \mu \bar{\phi}_{i}$, converges in $C(\bar{K})$. Now, repeating the same arguments as above, we can deduce an analogue of Theorem 5.8.

In the rest of this section, some examples are exhibited.

Example 5.1 Let $\Gamma=(V, E, r)$ be a connected, finite network and $|\Gamma|$ the metric graph associated to $\Gamma$. We take a sequence of Radon measures $\mu_{n}$ on $|\Gamma|$ with supp $\mu_{n}=|\Gamma|$ and suppose that $\mu_{n}$ vaguely converges to a measure $\mu$ such that $\mu=\sum_{v \in V} \pi(v)^{2} \delta_{v}$, where $\pi$ is a positive function on $V$. Then the limit of $\left(|\Gamma|, \mathcal{E}_{|\Gamma|}, \mu_{n}\right)$ as $n \rightarrow \infty$ in the sense of Theorem 5.8 is described as follows: $(Z, \mathcal{E}, \mu)=\left(V, \mathcal{E}_{\Gamma}, \mu\right)$, supp $\mu=V$ (and hence $\left.\mathcal{E}_{V}^{*}=\mathcal{E}_{\Gamma}\right)$ and the approximating maps are given by the identity map.

Example 5.2 Let $K_{n}=\left(V_{n}, E_{n}, r_{n}\right)$ be the complete graph of $n$ vertices, say $V_{n}=\{1,2, \ldots, n\}$, endowed with resistance $r=n / 2$. Then the effective resistance $R_{n}$ is equal to 1 . Let $\left(\mathcal{E}_{\infty}, D\left[\mathcal{E}_{\infty}\right]\right)$ be a quadratic form on $\mathbb{N} \cup\{\infty\}$ 
defined by

$$
\begin{gathered}
D\left[\mathcal{E}_{\infty}\right]=\left\{u \in \ell(\mathbb{N} \cup\{\infty\})\left|\sum_{i \in \mathbb{N}}\right| u(i)-\left.u(\infty)\right|^{2}<+\infty\right\} ; \\
\mathcal{E}_{\infty}(u)=2 \sum_{i \in \mathbb{N}}|u(i)-u(\infty)|^{2}, \quad u \in D[\mathcal{E}] .
\end{gathered}
$$

We assume that $V_{n}$ sits in $\mathbb{N} \cup\{\infty\}$ and consider the trace $\mathcal{E}_{\infty ; V_{n}}^{*}$ of $\mathcal{E}_{\infty}$ on $V_{n}$. Then the effective resistance of $\mathcal{E}_{\infty ; V_{n}}^{*}$ is equal to 1 and $\left(K_{n}, \mathcal{E}_{K_{n}}\right)$ can be identified with $\left(V_{n}, \mathcal{E}_{\infty ; V_{n}}^{*}\right)$. The limit of $\mathcal{E}_{\infty ; V_{n}}^{*}$ as $n \rightarrow \infty$ is given by the trace $\mathcal{E}_{\infty ; \mathbb{N}}^{*}$ of $\mathcal{E}_{\infty}$ on $\mathbb{N}$, that is

$$
\begin{gathered}
D\left[\mathcal{E}_{\infty ; \mathbb{N}}^{*}\right]=\left\{u \in \ell(\mathbb{N})\left|\sum_{i \in \mathbb{N}}\right| u(i)-\left.c\right|^{2}<+\infty \text { for some } c \in \mathbb{R}\right\} ; \\
\mathcal{E}_{\infty ; \mathbb{N}}^{*}(u)=2 \sum_{i \in \mathbb{N}}|u(i)-u(\infty)|^{2}, \quad u \in D\left[\mathcal{E}_{\mathbb{N}}^{*}\right]
\end{gathered}
$$

where $u(\infty)=\lim _{n \rightarrow \infty} \sum_{i \in V_{n}} u(i) / n$. Let $\pi$ be a positive function on $\mathbb{N}$ such that $\sum_{i \in \mathbb{N}} \pi(i)^{2}<+\infty$. Then $\left(V_{n}, \mathcal{E}_{\infty ; V_{n}}^{*}, \mu_{n}=\sum_{i \in V_{n}} \pi(i)^{2} \delta_{i}\right)$ converges to $\left(\mathbb{N}, \mathcal{E}_{\infty ; \mathbb{N}}^{*}, \mu=\sum_{i \in \mathbb{N}} \pi(i)^{2} \delta_{i}\right)$ as $n \rightarrow \infty$ in the sense of Theorem 5.8; the approximating maps are given by the inclusion maps.

Example 5.3 Let $G_{n}$ be a subgraph of the integer lattice $\mathbb{Z}^{d}$ generated by the set of vertices $V_{n}=\left\{\left(x_{1}, \ldots, x_{d}\right)|| x_{i} \mid \leq n, i=1, \ldots, d\right\}$. We consider the case where $d \geq 3$ and a sequence of measures $\mu_{n}$ on $V_{n}$ defined by $\mu_{n}(u)=\sum_{x \in V_{n}} u(x) \pi(x)^{2}\left(u \in \ell\left(V_{n}\right)\right)$, where $\pi$ is a positive function on $\mathbb{Z}^{d}$ such that $\sum_{x \in \mathbb{Z}^{d}} \pi(x)^{2}<+\infty$. Then we have a compact metric space $\left(\mathbb{Z}^{d} \cup\{\infty\}, \hat{S}, \mu\right)$ to which the sequence of $G_{n}$ with the measure $\mu_{n}$ converges in the sense of Theorem 5.8. However the effective resistance of $\mathbb{Z}^{d} \cup\{\infty\}$ provides it with the discrete topology.

Example 5.4 We consider Markov forms on a finite set $X$ and their $\Gamma$ limits. Let $\mathcal{M}$ be a family of Markov forms $\mathcal{E}$ on $\ell(X)$ such that the resistive networks associated to the forms $\mathcal{E}$ are connected and there exist functions $u$ satisfying $\mathcal{E}(u)=0$ and $\int_{X} u^{2} d \mu_{X}^{c}\left(=\sum_{x \in X} u(x)^{2}\right)=1$; such functions do not change their signs and here after we take the positive ones, denoted by $\pi_{\mathcal{E}}$. Associated to such a form $\mathcal{E}$, we have a Markov form $\hat{\mathcal{E}}$ defined by $\hat{\mathcal{E}}(u, v)=\mathcal{E}\left(u \pi_{\mathcal{E}}, v \pi_{\mathcal{E}}\right)$ for $u, v \in \ell(X)$, that satisfies [H-1] and [H-4].

We are now given a sequence $\left\{\mathcal{E}_{n}\right\}$ in $\mathcal{M}$ and suppose that as $n \rightarrow \infty$, the associated forms $\hat{\mathcal{E}}_{n} \Gamma$-converges to a form $\hat{\mathcal{E}}$ defined on a subspace $D[\hat{\mathcal{E}}]$ of $\ell(X)$ in the same manner described as in Theorem 4.5. We keep the notations there. Then $D[\hat{\mathcal{E}}]$ can be identified with the space of functions on 
the quotient space $X^{*}=X / \sim_{0}$ and the limit form $\hat{\mathcal{E}}$ will be also considered as a form on $\ell\left(X^{*}\right)$. According to the decomposition of $X, X=\cup_{\alpha=1, \ldots, p} X_{\alpha}$, relative to the equivalence relation $\sim_{b}, \hat{\mathcal{E}}$ splits into the sum of the forms $\hat{\mathcal{E}}_{\alpha}$ on $X_{\alpha}^{*}\left(=X_{\alpha} / \sim_{0}\right)$. Now we assume that the functions $\pi_{n}\left(=\pi_{\mathcal{E}_{n}}\right)$ on $X$ pointwise converges to a function $\pi$ on $X$ as $n \rightarrow \infty$, and define a function $\tilde{\pi}$ on $X^{*}$ by $\tilde{\pi}\left(x^{*}\right)=\sum_{x \in \rho^{-1}\left(x^{*}\right)} \pi(x)$ for $x^{*} \in X^{*}$. Let $\rho: X \rightarrow X^{*}$ be the canonical projection and $\hat{\mathcal{E}}_{\tilde{K}}^{*}$ the trace of $\hat{\mathcal{E}}$ on the support $\tilde{K}$ of the function $\tilde{\pi}$. For a function $v \in D\left[\hat{\mathcal{E}}_{\tilde{K}}^{*}\right]$, we assume that the minimizer $H_{\tilde{K} ; v}$ vanishes in the component $X_{\alpha}^{*}$ that does not intersect $\tilde{K}$. We define a linear map $S$ of $\ell\left(X^{*}\right)$ into $\ell(X)$ by $S(v)=\pi H_{\tilde{K}: v} \circ \rho$ for $v \in \ell\left(X^{*}\right)$. Then $S$ is injective, and a Markov form $(\mathcal{E}, D[\mathcal{E}])$ on $X$ is given by letting $\mathcal{E}(S(v))=$ $\hat{\mathcal{E}}_{\tilde{K}}^{*}(v)$ for $S(v)$ and $\mathcal{E}(u)=+\infty$ for a function $u$ that does not stay in the image of $S$. In what follows, we prove that the Markov forms $\mathcal{E}_{n}$ on $\ell(X)$ $\Gamma$-converges to $\mathcal{E}$ as $n \rightarrow \infty$. To do this, it suffices to verify that if $\mathcal{E}_{n} \Gamma$ converges to a form $\mathcal{E}^{\prime}$, then $\mathcal{E}^{\prime}=\mathcal{E}$. Let $v$ be a function on $\tilde{K}$. We take a sequence of functions $u_{n}$ on $X$ in such a way that $u_{n}$ converges to $H_{\tilde{K} ; v} \circ \rho$ pointwise on $X$ and $\hat{\mathcal{E}}_{n}\left(u_{n}\right)\left(=\mathcal{E}_{n}\left(\pi_{n} u_{n}\right)\right)$ tends to $\hat{\mathcal{E}}\left(H_{\tilde{K} ; v} \circ \rho\right)$. Since $\pi_{n} u_{n}$ converges to $S(v)=\pi H_{\tilde{K} ; v} \circ \rho$, we get

$$
\mathcal{E}^{\prime}(S(v)) \leq \liminf _{n \rightarrow \infty} \mathcal{E}_{n}\left(\pi_{n} u_{n}\right)=\lim _{n \rightarrow \infty} \hat{\mathcal{E}}_{n}\left(u_{n}\right)=\mathcal{E}(S(v)) .
$$

On the other hand, for $\phi \in D\left[\mathcal{E}^{\prime}\right]$, we have a sequence of functions $\phi_{n}$ on $X$ such that $\phi_{n}$ converges to $\phi$ pointwise in $X$ and $\mathcal{E}_{n}\left(\phi_{n}\right)$ tends to $\mathcal{E}^{\prime}(\phi)$ as $n \rightarrow \infty$. Here we may assume that $\phi_{n} / \pi_{n} \in L^{2}\left(X, \pi_{n}^{2} \mu^{c}\right) L^{2}$-strongly converges to $v \circ \rho \in L^{2}\left(X, \pi^{2} \mu^{c}\right)$ for some $v \in \ell(\tilde{K})$. Then we have $S(v)=\phi$ and

$$
\hat{\mathcal{E}}_{\widetilde{K}}^{*}(v) \leq \liminf _{n \rightarrow \infty} \hat{\mathcal{E}}_{n}\left(\phi_{n} / \pi_{n}\right)=\mathcal{E}^{\prime}(\phi) .
$$

Thus we have shown that $\mathcal{E}^{\prime}=\mathcal{E}$.

Now we illustrate a particular example of $\Gamma$-convergence of Markov forms described above. Let $(X, E, r)$ be a finite network and $\mathcal{E}$ the associated form on $\ell(X)$. Given a sequence of positive functions $\pi_{n}$ on $X$, we have a sequence of Markov forms $\mathcal{E}_{n}$ on $\ell(X)$ defined by

$$
\mathcal{E}_{n}(u, v)=\mathcal{E}\left(u / \pi_{n}, v / \pi_{n}\right), \quad u, v \in \ell(X) .
$$

Suppose that $\pi_{n}$ converges to a nonnegative function $\pi_{\infty}$ on $X$. Let $\mu_{n}$ (resp. $\mu_{\infty}$ ) be measures on $X$ given by $\mu_{n}(u)=\sum_{x \in X} u(x) \pi_{n}(x)^{2}$ (resp. $\left.\mu_{\infty}(u)=\sum_{x \in K} u(x) \pi_{\infty}(x)^{2}\right)$, where $K$ stands for the support of $\pi_{\infty}$. Then as $n \rightarrow \infty$, the symmetric operator of $L^{2}\left(V, \mu_{n}\right)$ associated to $\mathcal{E}$ converges to that of $L^{2}\left(K, \mu_{\infty}\right)$ associated to the trace $\hat{\mathcal{E}}_{K}^{*}$ on $K$ in the sense of Theorem 5.8. Moreover the form $\mathcal{E}_{n} \Gamma$-converges to a Markov form $\left(\mathcal{E}_{\infty}, D\left[\mathcal{E}_{\infty}\right]\right)$ on $\ell(X)$ defined by $D\left[\mathcal{E}_{\infty}\right]=\left\{H_{K ; u} \mid u \in \ell(K)\right\}$ and $\mathcal{E}_{\infty}\left(H_{K ; u}\right)=\mathcal{E}\left(H_{K ; u}\right)$. 


\section{Convergence of potentials and harmonic functions}

In this section, we consider a sequence of compact metric space $\left(X_{n}, R_{n}\right)$ of the resistance forms $\mathcal{E}_{n}$, and assume that it converges to a compact metric space $\left(Y, R_{Y}\right)$ in the Gromov-Hausdorff sense via approximating maps $f_{n}$ : $X_{n} \rightarrow Y$ as in Corollary 5.7. Let $\mathcal{E}_{Y}$ be the resistance form on $Y$ associated to the metric $R_{Y}$.

Let us begin with stating the following

Lemma 6.1 Let $\left\{u_{n}\right\}$ and $\left\{v_{n}\right\}$ be sequences of functions $u_{n}$ and $v_{n}$ in $D\left[\mathcal{E}_{n}\right]$ such that as $n \rightarrow \infty, \mathcal{E}_{n}\left(u_{n}\right)$ and also $\mathcal{E}_{n}\left(v_{n}\right)$ are bounded and furthermore $u_{n}$ and $v_{n}$ uniformly converge to continuous functions $u$ and $v$ on $Y$, respectively. Suppose that $\mathcal{E}_{n}\left(u_{n}\right)$ tends to $\mathcal{E}_{Y}(u)$ as $n \rightarrow \infty$. Then one has

$$
\mathcal{E}_{Y}(u, v)=\lim _{n \rightarrow \infty} \mathcal{E}_{n}\left(u_{n}, v_{n}\right) .
$$

See Lemma 3.4 in [26] for the proof of this lemma.

Now we show the following

Lemma 6.2 Suppose that a sequence of functions $u_{n} \in D\left[\mathcal{E}_{n}\right]$ uniformly converges to a function $u \in D\left[\mathcal{E}_{Y}\right]$ and $\mathcal{E}_{n}\left(u_{n}\right)$ tends to $\mathcal{E}_{Y}(u)$ as $n \rightarrow \infty$. Then a sequence of the energy measures $\mu_{\left\langle u_{n}\right\rangle}$ vaguely converges to the energy measure $\mu_{\langle u\rangle}$ of $u$ in the sense that

$$
\lim _{n \rightarrow \infty} \int f_{n}^{*} \phi d \mu_{\left\langle u_{n}\right\rangle}=\int \phi d \mu_{\langle u\rangle}, \quad \phi \in C(Y) .
$$

Proof. Since $D\left[\mathcal{E}_{Y}\right]$ is dense in $C(Y)$, it suffices to prove the lemma for $\phi \in D\left[\mathcal{E}_{Y}\right]$. Then we take a sequence of functions $\phi_{n} \in D\left[\mathcal{E}_{n}\right]$ in such a way that $\phi_{n}$ uniformly converges to $\phi$ and $\mathcal{E}_{n}\left(\phi_{n}\right)$ tends to $\mathcal{E}_{Y}(\phi)$ as $n \rightarrow \infty$. Then in view of Lemma 6.1, we see that

$$
\begin{aligned}
\lim _{n \rightarrow \infty} \int \phi_{n} d \mu_{\left\langle u_{n}\right\rangle} & =\lim _{n \rightarrow \infty} \mathcal{E}_{n}\left(\phi_{n} u_{n}, u_{n}\right)-\frac{1}{2} \mathcal{E}_{n}\left(\phi_{n}, u_{n}^{2}\right) \\
& =\mathcal{E}_{Y}(\phi u, u)-\frac{1}{2} \mathcal{E}_{Y}\left(\phi, u^{2}\right)=\int \phi d \mu_{\langle u\rangle} .
\end{aligned}
$$

Hence we get

$$
\lim _{n \rightarrow \infty} \int f_{n}^{*} \phi d \mu_{\left\langle u_{n}\right\rangle}=\lim _{n \rightarrow \infty} \int\left(f_{n}^{*} \phi-\phi_{n}\right) d \mu_{\left\langle u_{n}\right\rangle}+\int \phi_{n} d \mu_{\left\langle u_{n}\right\rangle}=\int \phi d \mu_{\langle u\rangle} .
$$

This completes the proof of Lemma 6.2.

We say that a sequence of closed subsets $K_{n}$ of $X_{n}$ converges to a closed subset $K$ of $Y$ if $f_{n}\left(K_{n}\right)$ converges to $K$ in $Y$ as $n \rightarrow \infty$. 
Lemma 6.3 Let $\left\{K_{n}\right\}$ be a sequence of closed subsets of $X_{n}$ which converges to a closed subset $K$ of $Y$ as $n \rightarrow \infty$. Then given $u \in D\left[\mathcal{E}_{Y} ; K\right]$, there exists a sequence of functions $u_{n} \in D\left[\mathcal{E}_{n} ; K_{n}\right]$ such that as $n \rightarrow \infty$, $u_{n}$ uniformly converges to $u$ and $\mathcal{E}_{n}\left(u_{n}\right)$ tends to $\mathcal{E}_{Y}(u)$.

Proof. For a positive number $a$, let $\Omega_{n}(-a)=\left\{x \in X_{n} \mid R_{n}\left(x, K_{n}\right)>a\right\}$ and $\Omega(-a)=\left\{x \in Y \mid R_{Y}(x, K)>a\right\}$. Given $u \in D\left[\mathcal{E}_{Y} ; K\right]$, we may assume that $u$ is supported in $\Omega(-a)$ for some $a>0$, because we can approximate $u$ by functions supported in $Y \backslash K$. Then we can take a sequence of functions $v_{n} \in D\left[\mathcal{E}_{n}\right]$ which uniformly converges to $u$ in such a way that $\mathcal{E}_{n}\left(v_{n}\right)$ tends to $\mathcal{E}_{Y}(u)$ as $n \rightarrow \infty$. Let $\varepsilon_{n}=2 \sup \left\{\left|v_{n}(x)\right| \mid x \in X_{n} \backslash \Omega_{n}(-a)\right\}$, and put $u_{n}=\max \left\{v_{n}^{+}, \varepsilon\right\}-\max \left\{v_{n}^{-}, \varepsilon\right\}$. Then $u_{n}$ vanishes on $K_{n}$ and uniformly converges to $u$ as $n \rightarrow \infty$, since $\varepsilon_{n}$ tends to 0 as $n \rightarrow \infty$. Moreover noting that $\mathcal{E}_{n}\left(u_{n}\right) \leq \mathcal{E}_{n}\left(v_{n}\right)$, we have

$$
\mathcal{E}_{Y}(u) \leq \liminf _{n \rightarrow \infty} \mathcal{E}_{n}\left(u_{n}\right) \leq \limsup _{n \rightarrow \infty} \mathcal{E}_{n}\left(v_{n}\right)=\mathcal{E}_{Y}(u) .
$$

This shows that $\lim _{n \rightarrow \infty} \mathcal{E}_{n}\left(u_{n}\right)=\mathcal{E}_{Y}(u)$, and the proof of the lemma is completed.

In Lemma 6.3, we assume further that $u \in D\left[\mathcal{E}_{Y} ; K\right]$ is nonnegative on a closed subset $L$ of $Y$ with $K \cap L=\emptyset$; then for a sequence of closed subsets $L_{n}$ of $X_{n}$ which converges to a closed subset $L$, we can find a sequence of functions $u_{n} \in D\left[\mathcal{E}_{n} ; K_{n}\right]$ as above with $u_{n} \geq 0$ on $L_{n}$.

Theorem 6.4 Given a sequence of proper closed subsets $K_{n}$ of $X_{n}$ which converges to a proper closed subset $K$ of $Y$, the Green function $g_{K_{n}}$ of $D\left[\mathcal{E}_{n} ; K_{n}\right]$ uniformly converges to the Green function $g_{K}$ of $D\left[\mathcal{E}_{Y} ; K\right]$ as $n \rightarrow \infty$, that is,

$$
\lim _{n \rightarrow \infty} \sup _{x, y \in X_{n}}\left|g_{K_{n}}(x, y)-g_{K}\left(f_{n}(x), f_{n}(y)\right)\right|=0 .
$$

Moreover let $\left\{\nu_{n}\right\}$ be a sequence of signed Radon measures $\nu_{n}$ on $X_{n}$ with $\sup _{n}\left|\nu_{n}\right|\left(X_{n}\right)<+\infty$, and suppose that $\nu_{n}$ vaguely converges to a signed Radon measure $\nu$ on $Y$. Then the function $U_{\nu_{n}}(x)=\nu_{n}\left(g_{K_{n}}(x, *)\right)\left(x \in X_{n}\right)$ uniformly converges to the function $U_{\nu}(x)=\nu\left(g_{K}(x, *)\right)(x \in Y)$, and $\mathcal{E}_{n}\left(U_{\nu_{n}}\right)$ tends to $\mathcal{E}_{Y}\left(U_{\nu}\right)$ as $n \rightarrow \infty$.

Proof. We assume that the diameter of $\left(K_{n}, R_{n}\right)$ is bounded from above by a positive constant $b$. Let $U_{n}=U_{\nu_{n}}$ and $U=U_{\nu}$ for simplicity. Then we have

$$
\left|U_{n}(x)\right| \leq \mathcal{E}_{n}\left(U_{n}\right)^{1 / 2} b^{1 / 2}, \quad x \in X_{n}
$$

and hence

$$
\mathcal{E}_{n}\left(U_{n}\right)=\nu_{n}\left(U_{n}\right) \leq \mathcal{E}_{n}\left(U_{n}\right)^{1 / 2} b^{1 / 2}\left|\nu_{n}\right|\left(X_{n}\right)
$$


These imply that $\mathcal{E}_{n}\left(U_{n}\right) \leq b\left|\nu_{n}\right|\left(X_{n}\right)^{2}$ and $\left|U_{n}\right| \leq b\left|\nu_{n}\right|\left(X_{n}\right)$. Therefore it follows from the compactness property of the convergence, 5.3 (iv), that there exists a subsequence $\left\{U_{k}\right\}$ which uniformly converges to a function $V \in D\left[\mathcal{E}_{Y}\right]$ as $k \rightarrow \infty$. For any $u \in D\left[\mathcal{E}_{Y} ; K\right]$, Lemma 6.3 allows us to take a sequence of functions $u_{n} \in D\left[\mathcal{E}_{n} ; K_{n}\right]$ which uniformly converges to $u$ in such a way that $\mathcal{E}_{n}\left(u_{n}\right)$ tends to $\mathcal{E}_{Y}(u)$. Then by Lemma 6.1 , we have

$\mathcal{E}_{Y}(V, u)=\lim _{k \rightarrow \infty} \mathcal{E}_{k}\left(U_{k}, u_{k}\right)=\lim _{k \rightarrow \infty} \nu_{n}\left(u_{k}\right)=\lim _{k \rightarrow \infty} \nu_{n}\left(u_{k}-f_{n}^{*} u\right)+\nu_{n}\left(f_{n}^{*} u\right)=\nu(u)$.

This shows that $V=U$, and hence $U_{n}$ uniformly converges to $U$ and furthermore

$$
\mathcal{E}_{Y}(U)=\nu(U)=\lim _{n \rightarrow \infty} \nu_{n}\left(U_{n}\right)=\lim _{n \rightarrow \infty} \mathcal{E}_{n}\left(U_{n}\right) .
$$

Let $\left\{x_{n}\right\}$ be a sequence of points $x_{n}$ of $X_{n}$ which converges to a point $x$ of $Y$ as $n \rightarrow \infty$. Then we apply the above result to the point measures $\delta_{x_{n}}$ and $\delta_{x}$ supported at $x_{n}$ and $x$ respectively, obtaining that $g_{K_{n}}\left(x_{n}, *\right)$ uniformly converges to $g_{K}(x, *)$ as $n \rightarrow \infty$. Thus we can conclude that $g_{K_{n}}$ uniformly converges to $g_{K}$ as $n \rightarrow \infty$. This completes the proof of Theorem 6.4.

Corollary 6.5 Let $\left\{\Omega_{n}\right\}$ be a sequence of proper, connected open subsets of $X_{n}$ which converges to a proper, connected open subset $\Omega$ of $Y$; let $\left\{L_{n}\right\}$ be a sequence of compact subsets of $\Omega_{n}$ which converges to a compact subset $L$ of $\Omega$. Then the sequence of the numbers $C\left(\Omega_{n}, L_{n}\right)$ relative to $\Omega_{n}$ and $L_{n}$ defined in Theorem 2.8 tends to the number $C(\Omega, L)$ relative to $\Omega$ and $L$ as $n \rightarrow \infty$. Moreover the equilibrium potential of $L_{n}$ in $\Omega_{n}, e_{\Omega_{n}, L_{n}}$, uniformly converges to that of $L$ in $\Omega$, e $e_{\Omega, L}$, in such a way that $\lim _{n \rightarrow \infty} \mathcal{E}_{n}\left(e_{\Omega_{n}, L_{n}}\right)=$ $\mathcal{E}_{Y}\left(e_{\Omega, L}\right)$, that is, the capacity of $L_{n}$ relative to $\Omega_{n}$ converges to that of $L$ relative to $\Omega$.

Proof. The first assertion is a direct consequence of Theorem 6.4. To prove the second one, let $e_{n}=e_{\Omega_{n}, L_{n}}$ and $e=e_{\Omega, L}$, and note that the capacity $\operatorname{Cap}_{\Omega_{n}}\left(L_{n}\right)$ of $L_{n}$ in $\Omega_{n}$ are uniformly bounded by (2.1). Therefore we can take a subsequence $\left\{e_{k}\right\}$ such that $e_{k}$ uniformly converges to a function $e^{\prime} \in$ $D\left[\mathcal{E}_{Y}\right]$. Clearly $e^{\prime}=1$ on $L$. Moreover for any $v \in D\left[\mathcal{E}_{Y} ; Y \backslash \Omega\right]$ with $v \geq 0$ on $L$, it follows that there exists a sequence of functions $v_{n} \in D\left[\mathcal{E}_{n} ; X_{n} \backslash \Omega_{n}\right]$ such that $v_{n} \geq 0$ on $L_{n}, v_{n}$ uniformly converges to $v$, and $\mathcal{E}_{n}\left(v_{n}\right)$ tends to $\mathcal{E}_{Y}(v)$ as $n \rightarrow \infty$. Since $\mathcal{E}_{n}\left(e_{n}, v_{n}\right) \geq 0$ for all $n$, we also have $\mathcal{E}_{Y}\left(e^{\prime}, v\right) \geq 0$. Thus the characterization of the equilibrium potentials allows us to conclude that $e^{\prime}=e$, that is, $e_{n}$ uniformly converges to $e$ as $n \rightarrow \infty$.

It remains to prove that $\lim _{n \rightarrow \infty} \mathcal{E}_{n}\left(e_{n}\right)=\mathcal{E}_{Y}(e)$. For this, we choose a sequence of functions $e_{n}^{\prime} \in D\left[\mathcal{E}_{n} ; X_{n} \backslash \Omega_{n}\right]$ which uniformly converges to $e$ 
and whose energy tends to that of $e$ as $n \rightarrow \infty$. By multiplying a suitable constant to $e_{n}$ which is close to 1 , we may assume that $e_{n}^{\prime} \geq 1$ on $L_{n}$. Therefore we have

$$
\mathcal{E}_{Y}(e) \leq \liminf _{n \rightarrow \infty} \mathcal{E}_{n}\left(e_{n}\right) \leq \liminf _{n \rightarrow \infty} \mathcal{E}_{n}\left(e_{n}^{\prime}\right) \leq \limsup _{n \rightarrow \infty} \mathcal{E}_{n}\left(e_{n}^{\prime}\right)=\mathcal{E}_{Y}(e) .
$$

Thus we see that $\mathcal{E}_{n}\left(e_{n}\right)$ tends to $\mathcal{E}_{Y}(e)$ as $n \rightarrow \infty$. This completes the proof of Corollary 6.5.

Letting

$$
e_{\Omega_{n}, L_{n}}(x)=\int g_{X_{n} \backslash \Omega_{n}}(x, y) d \nu_{\Omega_{n}, L_{n}}(y) ; e_{\Omega, \omega}(x)=\int g_{Y \backslash \Omega}(x, y) d \nu_{\Omega, L}(y)
$$

in Corollary 6.5, then we see that the measure $\nu_{\Omega_{n}, L_{n}}$ vaguely converges to the measure $\nu_{\Omega, L}$ as $n \rightarrow \infty$.

Let $\left\{L_{n}\right\}$ be a sequence of closed subsets of $X_{n}$ that converges to a closed subset $L$ as $n \rightarrow \infty$. By changing approximations $f_{n}: X_{n} \rightarrow Y$ slightly, we may assume that the restriction of $f_{n}$ to $L_{n}$ gives approximation of $L_{n}$ to $L$. Via these approximations, the trace $\mathcal{E}_{n ; L_{n}}^{*}$ on $L_{n} \Gamma$-converges to the trace $\mathcal{E}_{Y ; L}^{*}$ on $L$.

Moreover we have the following

Theorem 6.6 Let $\left\{L_{n}\right\}$ be a sequence of compact subsets of $X_{n}$ that converges to a compact subset $L$ of $Y$ as $n \rightarrow \infty$.

(i) Let $\left\{u_{n}\right\}$ be a sequence of functions in $D\left[\mathcal{E}_{n}\right]$ such that $u_{n}$ uniformly converges to a function $u \in D\left[\mathcal{E}_{Y}\right]$ and $\mathcal{E}_{n}\left(u_{n}\right)$ tends to $\mathcal{E}_{Y}(u)$ as $n \rightarrow \infty$. Let $H_{n}$ be a unique minimizer among functions with the same values as $u_{n}$ on $L_{n}$. Then $H_{n}$ uniformly converges to the minimizer $H$ for $u$ on $L$ in such a way that the energy measure of $H_{n}$ vaguely converges to that of $H$ in $Y$ as $n \rightarrow \infty$.

(ii) Suppose that each $\mathcal{E}_{n}$ is local. Then for a sequence of continuous functions $u_{n}$ on $X_{n}$ which uniformly converges to a continuous function $u$ on $Y$, the unique solution $H_{n}$ in Theorem 2.11 for $u_{n \mid L_{n}}$ uniformly converges to the unique solution $H$ for $u_{\mid L}$ and further the energy measure of $H_{n}$ in $X_{n} \backslash L_{n}$ vaguely converges to that of $H$ in $Y \backslash L$, that is, for any continuous function $\phi$ supported in $Y \backslash L$, one has

$$
\int \phi d \mu_{\langle H\rangle}=\lim _{n \rightarrow \infty} \int f_{n}^{*} \phi d \mu_{\left\langle H_{n}\right\rangle} .
$$

Proof. We prove the first assertion. Let $Q_{n}=u_{n}-H_{n} \in D\left[\mathcal{E}_{n} ; L_{n}\right]$ and $Q=u-H \in D\left[\mathcal{E}_{Y} ; L\right]$. Since $\mathcal{E}_{n}\left(Q_{n}\right)=\mathcal{E}_{n}\left(u_{n}\right)-\mathcal{E}_{n}\left(H_{n}\right)$ is bounded, we can find a subsequence $\left\{Q_{k}\right\}$ and a function $Q^{\prime} \in D\left[\mathcal{E}_{Y} ; L\right]$ such that $Q_{k}$ uniformly converges to $Q^{\prime}$ as $k \rightarrow \infty$. Let $H^{\prime}=u-Q^{\prime}$, to which $H_{k}=u_{k}-Q_{k}$ uniformly converges. 
For any $v \in D\left[\mathcal{E}_{Y} ; L\right]$, by Lemma 6.3 , we can take a sequence of functions $v_{n} \in D\left[\mathcal{E}_{n} ; L_{n}\right]$ in such a way that as $n \rightarrow \infty, v_{n}$ uniformly converges to $v$ and $\mathcal{E}_{n}\left(v_{n}\right)$ tends to $\mathcal{E}_{Y}(v)$. Then in view of Lemma 6.1 , we get

$$
\mathcal{E}_{Y}\left(H^{\prime}, v\right)=\lim _{k \rightarrow \infty} \mathcal{E}_{k}\left(H_{k}, v_{k}\right)=0 .
$$

This shows that $H=H^{\prime}$, and thus $H_{n}$ uniformly converges to $H$. Moreover we have

$$
\begin{aligned}
\mathcal{E}_{Y}(u) & =\mathcal{E}_{Y}(H)+\mathcal{E}_{Y}(Q) \leq \liminf _{n \rightarrow \infty} \mathcal{E}_{n}\left(H_{n}\right)+\liminf _{n \rightarrow \infty} \mathcal{E}_{n}\left(Q_{n}\right) \\
& \leq \limsup _{n \rightarrow \infty} \mathcal{E}_{n}\left(H_{n}\right)+\mathcal{E}_{n}\left(Q_{n}\right)=\limsup _{n \rightarrow \infty} \mathcal{E}_{n}\left(u_{n}\right)=\mathcal{E}_{Y}(u) .
\end{aligned}
$$

Thus we can conclude that $\lim _{n \rightarrow \infty} \mathcal{E}_{n}\left(H_{n}\right)=\mathcal{E}_{Y}(H)$. Now we prove the second assertion. Given a positive number $\varepsilon$, we take a function $v_{\varepsilon} \in D\left[\mathcal{E}_{Y}\right]$ such that $\sup _{Y}\left|u-v_{\varepsilon}\right|<\varepsilon$. Let $\left\{v_{n}\right\}$ be a sequence of functions in $D\left[\mathcal{E}_{n}\right]$ which uniformly converges to $v_{\varepsilon}$ and whose energy tends to that of $v_{\varepsilon}$. Let $H_{n}^{\prime}$ (resp. $H_{\varepsilon}$ ) be a unique minimizer in the set of functions with the same values as $v_{n}$ (resp. $v_{\varepsilon}$ ) on $L_{n}$ (resp. $L$ ). Then as we have just seen, $H_{n}^{\prime}$ uniformly converges to $H_{\varepsilon}$ and $\mathcal{E}_{n}\left(H_{n}^{\prime}\right)$ tends to $\mathcal{E}_{Y}\left(H_{\varepsilon}\right)$ as $n \rightarrow \infty$. Note that

$$
\sup _{X_{n}}\left|H_{n}^{\prime}-H_{n}\right| \leq \sup _{L_{n}}\left|v_{n}-u_{n}\right| \leq 2 \sup _{L}\left|v_{\varepsilon}-u\right|<2 \varepsilon
$$

for $n$ large enough. Hence we have

$$
\sup _{X_{n}}\left|H_{n}-f_{n}^{*} H\right| \leq \sup _{X_{n}}\left|H_{n}-H_{n}^{\prime}\right|+\sup _{X_{n}}\left|H_{n}^{\prime}-f_{n}^{*} H_{\varepsilon}\right|+\sup _{L_{n}}\left|f_{n}^{*} H_{\varepsilon}-f_{n}^{*} H\right|<4 \varepsilon
$$

for $n$ sufficiently large. Thus letting $\varepsilon \rightarrow 0$, we can conclude that $H_{n}$ uniformly converges to $H$.

It remains to prove that for any function $\phi \in C(Y)$ supported in $Y \backslash L$, $\int f^{*} \phi d \mu_{\left\langle H_{n}\right\rangle}$ tends to $\int \phi d \mu_{\langle H\rangle}$. To see this, we may assume that $\phi$ is of finite energy. Then we can take a sequence of functions $\phi_{n} \in D\left[\mathcal{E}_{n} ; L_{n}\right]$ such that each $\phi_{n}$ is supported in $\Omega_{n}(-4 a)\left(=\left\{x \in X_{n} \mid R_{n}\left(x, X_{n} \backslash \Omega_{n}\right)>4 a\right\}\right)$ for some positive constant $a$, and $\phi_{n}$ uniformly converges to $\phi$ as $n \rightarrow \infty$. Then applying Theorem 2.9, we get

$$
\begin{aligned}
\int\left|f_{n}^{*} \phi-\phi_{n}\right| d \mu_{\left\langle H_{n}\right\rangle} & \leq \sup _{\Omega_{n}^{\prime}(-3 a)}\left|f_{n}^{*} \phi-\phi_{n}\right| \int_{\Omega_{n}^{\prime}(-3 a)} d \mu_{\left\langle H_{n}\right\rangle} \\
& \leq \sup _{\Omega_{n}(-3 a)}\left|f_{n}^{*} \phi-\phi_{n}\right| \sup _{\Omega_{n}(-2 a)}\left|H_{n}\right|^{2} \operatorname{Cap}_{\Omega_{n}}\left(\Omega_{n}(-3 a)\right),
\end{aligned}
$$

and hence we obtain

$$
\lim _{n \rightarrow \infty} \int\left|f_{n}^{*} \phi-\phi_{n}\right| d \mu_{\left\langle H_{n}\right\rangle}=0
$$


For a positive number $\varepsilon$, let $H_{n}^{\prime}$ and $H_{\varepsilon}$ be as above and observe that

$$
\begin{aligned}
& \left|\int \phi_{n}\left(d \mu_{\left\langle H_{n}\right\rangle}-d \mu_{\left\langle H_{n}^{\prime}\right\rangle}\right)\right| \leq \\
& \leq \frac{\sup \left|\phi_{n}\right|}{2}\left(\frac{1}{\varepsilon} \int_{\Omega_{n}(-3 a)} d \mu_{\left\langle H_{n}-H_{n}^{\prime}\right\rangle}+\varepsilon \int_{\Omega_{n}(-3 a)} d \mu_{\left\langle H_{n}+H_{n}^{\prime}\right\rangle}\right) \\
& \leq \frac{\sup \left|\phi_{n}\right|}{2}\left(\frac{1}{\varepsilon} \int_{\Omega_{n}^{\prime}(-a)} d \mu_{\left\langle H_{n}-H_{n}^{\prime}\right\rangle}+2 \varepsilon\left(\int_{\Omega_{n}(-3 a)} d \mu_{\left\langle H_{n}\right\rangle}+\int_{\Omega_{n}(-3 a)} d \mu_{\left\langle H_{n}^{\prime}\right\rangle}\right)\right) \\
& \leq \frac{\sup \left|\phi_{n}\right|}{2} \operatorname{Cap}_{\Omega_{n}}\left(\Omega_{n}(-3 a)\right)\left(\frac{1}{\varepsilon} \sup _{\Omega_{n}}\left|H_{n}-H_{n}^{\prime}\right|^{2}+2 \varepsilon \sup _{\Omega_{n}}\left(\left|H_{n}\right|^{2}+\left|H_{n}^{\prime}\right|^{2}\right)\right) \\
& \leq \frac{\sup \left|\phi_{n}\right|}{2} \operatorname{Cap}_{\Omega_{n}}\left(\Omega_{n}(-3 a)\right)\left(\frac{1}{\varepsilon} \sup _{L_{n}}\left|u_{n}-u_{n}^{\prime}\right|^{2}+2 \varepsilon \sup _{L_{n}}\left(\left|u_{n}\right|^{2}+\left|u_{n}^{\prime}\right|^{2}\right)\right) .
\end{aligned}
$$

Hence we can deduce that

$$
\left|\int \phi_{n}\left(d \mu_{\left\langle H_{n}\right\rangle}-d \mu_{\left\langle H_{n}\right\rangle}\right)\right| \leq b \operatorname{Cap}_{\Omega_{n}}\left(\Omega_{n}(-3 a)\right) \varepsilon
$$

for all large $n$ and some constant $b$ independent of $n$ and $\varepsilon$. By the same reason, we have

$$
\left|\int \phi\left(d \mu_{\langle H\rangle}-d \mu_{\left\langle H_{\varepsilon}\right\rangle}\right)\right| \leq b \operatorname{Cap}_{\Omega}(\Omega(-3 a)) \varepsilon .
$$

These estimates imply that for all large $n$,

$$
\begin{aligned}
\left|\int \phi_{n} d \mu_{\left\langle H_{n}\right\rangle}-\int \phi d \mu_{\langle H\rangle}\right| & \leq 2 b \operatorname{Cap}_{\Omega}(\Omega(-a)) \varepsilon+\left|\int \phi_{n} d \mu_{\left\langle H_{n}^{\prime}\right\rangle}-\int \phi d \mu_{\left\langle H_{\varepsilon}\right\rangle}\right| \\
& \leq\left(4 b \operatorname{Cap}_{\Omega}(\Omega(-a))+1\right) \varepsilon .
\end{aligned}
$$

Letting $\varepsilon \rightarrow 0$, we can conclude that

$$
\lim _{n \rightarrow \infty} \int f_{n}^{*} \phi d \mu_{\left\langle H_{n}\right\rangle}=\lim _{n \rightarrow \infty} \int \phi_{n} d \mu_{\left\langle H_{n}\right\rangle}=\int \phi d \mu_{\langle H\rangle} .
$$

This completes the proof of Theorem 6.6.

Corollary 6.7 Let $\left\{\left(X_{n}, R_{n}\right)\right\}$ be a sequence of compact metric spaces of resistance forms $\mathcal{E}_{n}$ and suppose that it converges to a compact metric space $\left(Y, R_{Y}\right)$ with respect to the Gromov-Hausdorff distance. Suppose that each $\mathcal{E}_{n}$ is local. Then one has the following assertions:

(i) Let $H$ be a harmonic function on an open subset $\Omega$ of $Y$. Then there exists a sequence of open subsets $\Omega_{n}$ of $G_{n}$ which converges to $\Omega$ as $n \rightarrow \infty$, 
and a sequence of $\mathcal{E}_{n}$-harmonic functions $H_{n}$ on $\Omega_{n}$ such that $H_{n}$ uniformly converges to $H$ on each compact subset of $\Omega$ and the energy measure of $H_{n}$ vaguely converges to that of $H$ in $\Omega$ as $n \rightarrow \infty$.

(ii) Let $\left\{\Omega_{n}\right\}$ be a sequence of open subsets of $X_{n}$ which converges to an open subset $\Omega$ of $Y$. Let $\left\{H_{n}\right\}$ be a sequence of $\mathcal{E}_{n}$-harmonic functions on $\Omega_{n}$ and suppose that $H_{n}\left(o_{n}\right)$ is uniformly bounded as $n \rightarrow \infty$ for a sequence of points $o_{n} \in \Omega_{n}$ which converges to a point of $\Omega$. Then there exists a subsequence $\left\{H_{k}\right\}$ and an $\mathcal{E}_{Y}$-harmonic function $H$ on $\Omega$ such that $H_{k}$ uniformly converges to $H$ on each compact subset of $\Omega$ and the energy measure of $H_{k}$ vaguely converges to that of $H$ in $\Omega$ as $n \rightarrow \infty$.

We refer to [27] for related results to those in this section.

\section{Resistance forms on infinite networks}

Let $\Gamma=(V, E, r)$ be a connected, infinite network. In this section, we consider, besides $\mathcal{E}_{\Gamma}^{0}$ and $\mathcal{E}_{\Gamma}$, a resistance form $(\mathcal{E}, D[\mathcal{E}])$ on $\ell(V)$ such that $D_{0}\left[\mathcal{E}_{\Gamma}^{0}\right] \subset D[\mathcal{E}] \subset D\left[\mathcal{E}_{\Gamma}\right], \mathcal{E}(u) \geq \mathcal{E}_{\Gamma}(u)$ for all $u \in D[\mathcal{E}]$, and $\mathcal{E}(u, v)=$ $\mathcal{E}_{\Gamma}(u, v)$ for all $u \in D[\mathcal{E}]$ and $v \in D_{0}\left[\mathcal{E}_{\Gamma}\right]$ (cf. Theorem 5.3). We call such $\mathcal{E}$ a resistance form on $\Gamma$.

7.1 We consider two canonical measures on $V$, the counting measure $\mu_{V}^{c}=$ $\sum_{x \in V} \delta_{x}$ and the measure $\mu_{V}^{p}$ defined by $\mu_{V}^{p}=\sum_{x \in V} \pi_{V}(x) \delta_{x}$, where $\pi_{V}(x)=$ $\sum_{y \sim x} r(|x y|)^{-1}$. With respect to these measures, we have two spectral gaps of $\Gamma$ defined by

$$
\begin{aligned}
& \lambda_{0}^{c}(\Gamma)=\inf \left\{\frac{\mathcal{E}_{\Gamma}(u)}{\int u^{2} d \mu_{V}^{c}} \mid u \in \ell_{0}(V)\right\}, \\
& \lambda_{0}^{p}(\Gamma)=\inf \left\{\frac{\mathcal{E}_{\Gamma}(u)}{\int u^{2} d \mu_{V}^{p}} \mid u \in \ell_{0}(V)\right\} .
\end{aligned}
$$

For a resistance form $\mathcal{E}$ under consideration, we can define two nonnegative numbers $\lambda_{1}^{*}\left(\mathcal{E} ; \mu_{V}^{c}\right)$ and $\lambda_{1}^{*}\left(\mathcal{E} ; \mu_{V}^{p}\right)$ associated to the measures $\mu_{V}^{c}$ and $\mu_{V}^{p}$, respectively (cf. Definitions 3.1 and 3.2). We compare them in the following

Proposition 7.1 Under the above notations, it holds that $\lambda_{1}^{*}\left(\mathcal{E} ; \mu_{V}^{c}\right) \leq \lambda_{0}^{c}(\Gamma)$ and $\lambda_{1}^{*}\left(\mathcal{E} ; \mu_{V}^{p}\right) \leq \lambda_{0}^{p}(\Gamma)$. Moreover in the case where $\lambda_{0}^{c}(\Gamma)>0$, the following are mutually equivalent:

(i) $\lambda_{1}^{*}\left(\mathcal{E} ; \mu_{V}^{c}\right)>0$

(ii) $(\mathcal{E}, D[\mathcal{E}])=\left(\hat{\mathcal{E}}_{\Gamma}^{0}, \mathbb{R}+D_{0}\left[\mathcal{E}_{\Gamma}\right]\right)$,

(iii) $\lambda_{1}^{*}\left(\mathcal{E} ; \mu_{V}^{c}\right)=\lambda_{0}^{c}(\Gamma)$. 
Similarly in the case where $\lambda_{0}^{p}(\Gamma)>0$, the following are mutually equivalent:

(i) $\lambda_{1}^{*}\left(\mathcal{E} ; \mu_{V}^{p}\right)>0$,

(ii) $(\mathcal{E}, D[\mathcal{E}])=\left(\hat{\mathcal{E}}_{\Gamma}^{0}, \mathbb{R}+D_{0}\left[\mathcal{E}_{\Gamma}\right]\right)$,

(iii) $\lambda_{1}^{*}\left(\mathcal{E} ; \mu_{V}^{p}\right)=\lambda_{0}^{p}(\Gamma)$.

Proof. Let $u$ be a finitely supported function on $V$. Then $\mathcal{E}_{\Gamma}(u)=\mathcal{E}_{V_{n}}^{*}\left(u_{\mid V_{n}}\right)$ for large $n$, where $\left\{V_{n}\right\}$ is an exhaustion of $V$, and hence we have

$$
\mathcal{E}_{\Gamma}(u) \geq \lambda_{1}\left(\mathcal{E}_{V_{n}}^{*} ; \mu_{V_{n}}^{c}\right) \int_{V_{n}}\left(u-\mathcal{H}_{\mu_{V_{n}}^{c}} u\right)^{2} d \mu_{V_{n}}^{c}
$$

Letting $n \rightarrow \infty$, we obtain

$$
\mathcal{E}_{\Gamma}(u) \geq \lambda_{1}\left(\mathcal{E} ; \mu_{V}^{c}\right) \int_{V} u^{2} d \mu_{V}^{c} .
$$

This shows that $\lambda_{0}^{c}(\Gamma) \geq \lambda_{1}^{*}\left(\mathcal{E} ; \mu_{V}^{c}\right)$.

We consider the case where $\lambda_{0}^{c}(\Gamma)>0$. It follows from Theorem 3.5 that (i) implies (ii). Suppose that (ii) holds. Then for any $n$, we take a function $u_{n}$ on $V_{n}$ such that $\mathcal{H}_{\mu_{V_{n}}^{c}} u_{n}=0, \int_{V_{n}} u_{n}^{2} d \mu_{V_{n}}^{c}=1$ and $\mathcal{E}_{V_{n}}^{*}\left(u_{n}\right)=$ $\lambda_{1}^{*}\left(\mathcal{E}_{V_{n}}^{*} ; \mu_{V_{n}}^{c}\right)$. Let $H_{n}=H_{V_{n} ; u_{n}} \in D[\mathcal{E}]$ (see Lemma 1.6). Then by the assumption, $H_{n}-c_{n} \in D_{0}\left[\mathcal{E}_{\Gamma}\right]$ for some constant $c_{n}$. Hence we have

$$
\begin{aligned}
\lambda_{1}^{*}\left(\mathcal{E}_{V_{n}}^{*} ; \mu_{V_{n}}^{c}\right)=\mathcal{E}\left(H_{n}\right) & \geq \lambda_{0}^{c}(\Gamma) \int_{V}\left|H_{n}-c_{n}\right|^{2} d \mu_{V}^{c} \\
& \geq \lambda_{0}^{c}(\Gamma) \int_{V_{n}}\left|H_{n}-c_{n}\right|^{2} d \mu_{V_{n}}^{c} \\
& \geq \lambda_{0}^{c}(\Gamma) \int_{V_{n}} u_{n}^{2} d \mu_{V_{n}}^{c} \\
& \geq \lambda_{0}^{c}(\Gamma) .
\end{aligned}
$$

Letting $n \rightarrow \infty$, we get $\lambda_{1}^{*}\left(\mathcal{E} ; \mu_{V}^{c}\right) \geq \lambda_{0}^{c}(\Gamma)$, so that the equality holds. Obviously (iii) implies (i). The same arguments are valid for the case of $\lambda_{0}^{p}(\Gamma)$ and $\lambda_{1}^{*}\left(\mathcal{E} ; \mu_{V}^{p}\right)$. This completes the proof of Proposition 7.1.

Corollary 7.2 Let $\Gamma_{n}=\left(V_{n}, E_{n}, r_{n}\right)$ and $\Gamma=(V, E, r)$ be as in Theorem 5.3. Then

$$
\limsup _{n \rightarrow \infty} \lambda_{1}\left(\mathcal{E}_{\Gamma_{n}} ; \mu_{V_{n}}^{c}\right) \leq \lambda_{0}^{c}(\Gamma) \quad \text { and } \quad \limsup _{n \rightarrow \infty} \lambda_{1}\left(\mathcal{E}_{\Gamma_{n}} ; \mu_{V_{n}}^{p}\right) \leq \lambda_{0}^{p}(\Gamma) .
$$

It is known (cf. e.g., [39]) that $\lambda_{0}^{p}(G) \leq 1-2 \sqrt{d-1} / d$ and $\lambda_{0}^{c}(G) \leq$ $d-2 \sqrt{d-1}$ for an infinite graph $G=(V, E)$ with degree bounded by a positive integer $d$. Thus we have the following 
Theorem 7.3 (Alon-Boppana, Grigorchuk-Żuk [22]) Let $G_{n}=\left(V_{n}, E_{n}\right)$ be a sequence of finite graphs such that the degrees of $G_{n}$ are bounded by a positive integer $d$ and the cardinality of $V_{n}$ tends to infinity as $n \rightarrow \infty$. Then one has

$\limsup _{n \rightarrow \infty} \lambda_{1}\left(\mathcal{E}_{G_{n}} ; \mu_{V_{n}}^{c}\right) \leq d-2 \sqrt{d-1}$ and $\limsup _{n \rightarrow \infty} \lambda_{1}\left(\mathcal{E}_{G_{n}} ; \mu_{V_{n}}^{p}\right) \leq 1-2 \frac{\sqrt{d-1}}{d}$.

As a consequence of Proposition 7.1, together with Example 1.2, we have the following

Corollary 7.4 Let $\left\{G_{n}=\left(V_{n}, E_{n}\right)\right\}$ be a sequence of finite graphs such that the degrees of $G_{n}$ are bounded by a positive integer $d$ and $\lambda_{1}\left(\mathcal{E}_{G_{n}} ; \mu_{V_{n}}^{c}\right)$ is bounded away from zero by a positive constant. Let $T=\left(V_{T}, E_{T}\right)$ be the homogeneous tree of degree $d$. Suppose that a pointed metric space $\left(V_{n}, d_{G_{n}}, p_{n}\right)$ converges to a pointed metric space $\left(V_{T}, d_{T}, p\right)$ as $n \rightarrow \infty$. Then one has

$$
\lim _{n \rightarrow \infty} \sup _{x, y \in B_{\rho}\left(p_{n}\right)}\left|R_{G_{n}}(x, y)-\frac{2(d-1)}{d(d-2)}\left(1-\left(\frac{1}{d-1}\right)^{d_{G_{n}}(x, y)}\right)\right|=0
$$

for any $\rho>0$.

Let $G=(V, E)$ be a connected, finite graph. The girth of $G$ is by definition the length of the shortest circuit in $G$. We consider a family of connected finite graphs $G_{n}=\left(V_{n}, E_{n}\right)$ as in Corollary 7.4 satisfying further the property that the girth of $G_{n}$ diverges as $n \rightarrow \infty$. It is known (cf. [17] and the references therein) that there exists such a family $\left\{G_{n}\right\}$ with $\lim _{n \rightarrow \infty} \lambda_{1}\left(\mu_{V_{n}}^{c}\right)=d-2 \sqrt{d-1}$ for certain $d$ 's. As we have seen, for such a family, the resistance metric of $G_{n}$ converges to the minimal one of the homogeneous tree $T$ of degree $d$ as $n \rightarrow \infty$. Moreover using this family and modifying it appropriately, we can construct another family $\left\{G_{n}^{\prime}\right\}$ converging to $T$ with respect to the geodesic distances such that the limit resistance form $\mathcal{E}$ on $T$ is different from the minimal one; for example, the dimension of the space $H D\left[\mathcal{E}_{T}\right] \cap D[\mathcal{E}]$ is finite and equal to any given positive integer.

7.2 Let $\mathcal{E}$ be a resistance form on a connected infinite network $\Gamma=(V, E, r)$. We denote by $g_{z}^{\mathcal{E}}(x, y)$ and $R_{\mathcal{E}}$, respectively, the Green function and the effective resistance of $\mathcal{E}$. Set $H_{\mathcal{E}}=H D\left[\mathcal{E}_{\Gamma}\right] \cap D[\mathcal{E}]$. For $x, y, z \in V$, let

$$
h_{z}^{\mathcal{E}}(x, y)=g_{z}^{\mathcal{E}}(x, y)-\left(g_{\Gamma}^{0}(x, y)-g_{\Gamma}^{0}(x, z)-g_{\Gamma}^{0}(y, z)+g_{\Gamma}^{0}(z, z)\right) .
$$

Then $h_{z}^{\mathcal{E}}(x, y)=h_{z}^{\mathcal{E}}(y, x)$ and the functions $h_{z}^{\mathcal{E}}(x, y)$ of $y$ belongs to $H_{\mathcal{E}}$ and satisfies $h_{z}^{\mathcal{E}}(x, z)=0, h_{z}^{\mathcal{E}}(x, x)=R_{\mathcal{E}}(x, z)-R_{\Gamma}^{0}(x, y),-R_{\Gamma}^{0}(x, z) \leq h_{z}^{\mathcal{E}}(x, y) \leq$ $R_{\mathcal{E}}(x, z)$ for all $y \in V$, where we put $R_{\Gamma}^{0}(x, y)=g_{\Gamma}^{0}(x, x)-2 g_{\Gamma}^{0}(x, y)+g_{\Gamma}^{0}(z, z)$. In fact, $h_{z}^{\mathcal{E}}(x, y)$ is the Green function of the form $\mathcal{E}$ restricted to $H_{\mathcal{E}}$, that is, $\mathcal{E}\left(h_{z}(x, *), h\right)=h(x)-h(z)$ for any $h \in H_{\mathcal{E}}$. 
Let $N=\operatorname{dim} H_{\mathcal{E}}-1(\leq+\infty)$. In what follows, we assume that $1 \leq$ $N \leq+\infty$, and fix a point $o \in V$. Let $\left\{h_{i} \mid 1 \leq i<N+1\right\}$ be a complete orthonormal system of the Hilbert space $\left(\left\{h \in H_{\mathcal{E}} \mid h(o)=0\right\}, \mathcal{E}\right)$. Then it holds that

$$
\begin{aligned}
& h_{z}^{\mathcal{E}}(x, y)=\sum_{i=1}^{N}\left(h_{i}(x)-h_{i}(z)\right)\left(h_{i}(y)-h_{i}(z)\right), \quad x, y, z \in V \\
& h_{z}^{\mathcal{E}}(x, x)=R_{\mathcal{E}}(x, z)-R_{\Gamma}^{0}(x, z)=\sum_{i=1}^{N}\left(h_{i}(x)-h_{i}(z)\right)^{2}, \quad x, z \in V
\end{aligned}
$$

(cf. Lemma 1.11).

Suppose here that $\Gamma$ is locally finite. Let $\bar{R}_{\mathcal{E}}(x)=\sum_{y \sim x} R_{\mathcal{E}}(x, y) / r(x, y)$ and $\bar{R}_{\Gamma}^{0}(x)=\sum_{y \sim x} R_{\Gamma}^{0}(x, y) / r(x, y)$ for $x \in V$ (cf. [37], p. 98). Then we have

$$
\bar{R}_{\mathcal{E}}(x)-\bar{R}_{\Gamma}^{0}(x)=\sum_{i=1}^{N} \sum_{y \sim x} \frac{\left(h_{i}(x)-h_{i}(y)\right)^{2}}{r(x, y)}, \quad x \in V .
$$

It follows from the maximum principle that the set $\left\{x \in V \mid \bar{R}_{\mathcal{E}}(x)-\right.$ $\left.\bar{R}_{\Gamma}^{0}(x)>0\right\}$ is empty (resp. an infinite subset) if and only if $\operatorname{dim} H_{\mathcal{E}}=1$ (resp. $\operatorname{dim} H_{\mathcal{E}}>1$ ). Thus we arrive at the following

Proposition 7.5 Let $(\mathcal{E}, D[\mathcal{E}])$ be a resistance form on a connected, locally finite, infinite network $\Gamma$. Then one has

$$
\frac{1}{2} \sum_{x \in V} \bar{R}_{\mathcal{E}}(x)-\bar{R}_{\Gamma}^{0}(x) \leq \operatorname{dim} H_{\mathcal{E}}-1(\leq+\infty),
$$

and in the case where $\mathcal{E}=\mathcal{E}_{\Gamma}$,

$$
\frac{1}{2} \sum_{x \in V} \bar{R}_{\Gamma}(x)-\bar{R}_{\Gamma}^{0}(x)=\operatorname{dim} H D\left[\mathcal{E}_{\Gamma}\right]-1(\leq+\infty) .
$$

Let $\mathcal{E}$ be a resistance form on $\Gamma$. For a bijective map $\phi: V \rightarrow V$, it is easy to see that $\phi$ preserves the effective resistance of $\mathcal{E}$, that is, $R_{\mathcal{E}}(\phi(x), \phi(y))=R_{\mathcal{E}}(x, y)$ for all $x, y \in V$ if and only if $\phi$ satisfies the property that $\mathcal{E}\left(\phi^{*} u\right)=\mathcal{E}(u)$ for all $u \in D[\mathcal{E}]$. We remark that such a map $\phi$ must be an automorphism of the network $\Gamma=(V, E, r)$, since $\mathcal{E}$ coincides with $\mathcal{E}_{\Gamma}^{0}$ on $D_{0}\left[\mathcal{E}_{\Gamma}^{0}\right]$. We denote by $\operatorname{Aut}(\Gamma, \mathcal{E})$ the group of all automorphisms of $\Gamma$ preserving the effective resistance of $\mathcal{E}$. Then it is evident that $\bar{R}_{\mathcal{E}}-\bar{R}_{\Gamma}^{0}$ is constant on an orbit of $\operatorname{Aut}(\Gamma, \mathcal{E})$ on $V$. This shows the following

Corollary 7.6 Let $\Gamma$ and $(\mathcal{E}, D[\mathcal{E}])$ be as in Proposition 7.5. Suppose that there exists an orbit $K$ of $\operatorname{Aut}(\Gamma, \mathcal{E})$ such that $\bar{R}_{\mathcal{E}}-\bar{R}_{\Gamma}^{0}$ is positive on $K$ and the cardinality of $K$ is infinite. Then $H_{\mathcal{E}}$ is of infinite dimension. 
7.3 In this part, we study the compactification described in 3.2 with respect to a resistance form $\mathcal{E}$ of a connected, infinite network $\Gamma=(V, E, r)$. In what follows, we assume that $\Gamma$ is nonparabolic, that is, there exists the minimal Green function $g_{\Gamma}^{0}(x, y)$.

To begin with, we recall the following

Lemma 7.7 (Green's formula) Let $u$ be a function in $D\left[\mathcal{E}_{\Gamma}\right]$. Then for any function $g \in D_{0}\left[\mathcal{E}_{\Gamma}\right]$, one has

$$
\mathcal{E}_{\Gamma}(u, g)=\int_{V} g \mathcal{L}^{c} u d \mu_{V}^{c} \quad \text { if } g \mathcal{L}^{c} u \in L^{1}\left(V, \mu_{V}^{c}\right) .
$$

Proof. The identity holds if $g$ is finitely supported. For $g \in D_{0}\left[\mathcal{E}_{\Gamma}\right]$, we can take a sequence of finitely supported functions $g_{n}$ such that $\left|g_{n}\right| \leq|g|$ and $g_{n}$ converges to $g$ in $D\left[\mathcal{E}_{\Gamma}\right]$. Since $\left|g_{n} \mathcal{L}^{c} u\right| \leq\left|g \mathcal{L}^{c} u\right|$, Lebesgue's convergence theorem implies that $\lim _{n \rightarrow \infty} \int_{V} g_{n} \mathcal{L}^{c} u d \mu_{V}^{c}=\int_{V} g \mathcal{L}^{c} u d \mu_{V}^{c}$. This shows the lemma.

Let $\mathcal{E}$ be a resistance form on $\Gamma$. Associated to the space of bounded functions in $D[\mathcal{E}]$, we have a compactification of $V$, which we will call the Royden compactification $\mathcal{R}(V, \mathcal{E})$ of $\mathcal{E}$. The boundary $\partial R(V, \mathcal{E})=R(V, \mathcal{E}) \backslash$ $V$ will be called the Royden boundary of $\mathcal{E}$. The harmonic boundary of $\mathcal{E}$ is defined by $\Delta(\mathcal{E})=\left\{x \in \partial R(V, \mathcal{E}) \mid g(x)=0, \quad \forall g \in D_{0}\left[\mathcal{E}_{\Gamma}\right]\right\}$. We recall a basic fact concerning Dirichlet problems on the Royden boundary $\partial \mathcal{R}(V, \mathcal{E})$ ( cf. e.g., [37, Chap. VI]): for any continuous function $f$ on $\partial \mathcal{R}(V, \mathcal{E})$, there exists a unique harmonic function $H_{f}$ on $\Gamma$ such that for any $\xi \in \Delta(\mathcal{E})$, $\lim _{x \in V \rightarrow \xi} H_{f}(x)=f(\xi)$, and $\sup _{V}\left|H_{f}\right| \leq \max _{\Delta(\mathcal{E})}|f|$. Given a point $a \in V$, letting $\nu_{a}(f)=H_{f}(a)$ for $f \in C(\partial \mathcal{R}(V, \mathcal{E}))$, we have a Radon measure $\nu_{a}$ on $\partial \mathcal{R}(V, \mathcal{E})$, called the harmonic measure with respect to the point $a$. In view of the Harnack inequality, $\nu_{a}$ and $\nu_{b}$ are mutually absolutely continuous for any pair of points $a, b \in V$, and the harmonic measures are supported on the harmonic boundary of $\mathcal{E}$.

Let $\mu$ be a finite measure supported on $V$ such that

$$
\iint_{V \times V} R_{\mathcal{E}}(x, y)^{2} d \mu(x) d \mu(y)<+\infty .
$$

Condition (3.12) is automatically satisfied in this case.

In what follows, to indicate the dependency on the form $\mathcal{E}$, we denote by $\mathcal{N}_{\mathcal{E} ; \mu}, \bar{V}^{N}(\mathcal{E})$ and $\mathcal{L}_{\mathcal{E} ; \mu}$, respectively, the distance on $V$ introduced in 3.2, the completion of the metric space $\left(V, \mathcal{N}_{\mathcal{E} ; \mu}\right)$ and the infinitesimal generator of the form $\mathcal{E}$ in $L^{2}(V, \mu)$. Let $\partial \bar{V}^{N}(\mathcal{E})=\bar{V}^{N}(\mathcal{E}) \backslash V$. We will call $\bar{V}^{N}(\mathcal{E})$ and $\partial \bar{V}^{N}(\mathcal{E})$, respectively, the Kuramochi compactification and the Kuramochi 
boundary of a resistance form $\mathcal{E}$. A function of $D\left[\mathcal{L}_{\mathcal{E} ; \mu}\right]$ is regarded as a continuous function on $\bar{V}^{N}(\mathcal{E}$ ) (see Lemma 3.8 and Theorem 3.10). Given $u \in D\left[\mathcal{L}_{\mathcal{E} ; \mu}\right]$, we denote by $T_{\mathcal{E}} u$ the restriction of $u$ to the boundary $\partial \bar{V}^{N}(\mathcal{E})$. The identity map of $V$ extends to a continuous map from $\mathcal{R}(V, \mathcal{E})$ onto $\bar{V}^{N}(\mathcal{E})$. We denote by $\rho$ the induced map from $\partial \mathcal{R}(V, \mathcal{E})$ onto $\partial \bar{V}^{N}(\mathcal{E})$. Let $\Delta^{N}(\mathcal{E})=\rho(\Delta(\mathcal{E}))$ and $\omega_{a}=\rho_{*} \nu_{a}(a \in V)$. Here and after, we fix a point $o \in V$ and write $\omega$ for $\omega_{o}$.

Now we study Neumann problems on $\partial \bar{V}^{N}(\mathcal{E})$, referring to [18] and [34] where analysis on Green spaces are carried out.

Lemma 7.8 For any $u \in D\left[\mathcal{L}_{\mathcal{E} ; \mu}\right]$ and $a \in V$, one has

$$
\int_{\partial \bar{V}^{N}(\mathcal{E})}\left(T_{\mathcal{E}} u\right)^{2} d \omega_{a} \leq 2 g_{\Gamma}^{0}(a, a) \mathcal{E}(u)+2 u(a)^{2} .
$$

Proof. Put $f=T_{\mathcal{E}} u \in C\left(\partial \bar{V}_{\mathcal{E}}^{N}\right)$. We first observe that

$$
\mathcal{L}^{c}\left(H_{f^{2}}-\left(H_{f}\right)^{2}\right)(x)=\sum_{y \sim x} r(|x y|)^{-1}\left(H_{f}(x)-H_{f}(y)\right)^{2}, \quad x \in V,
$$

which implies

$$
\int_{V} \mathcal{L}^{c}\left(H_{f^{2}}-\left(H_{f}\right)^{2}\right) d \mu_{V}^{c}=2 \mathcal{E}_{\Gamma}\left(H_{f}\right) .
$$

Since $\left(H_{f}\right)^{2}-H_{f^{2}}$ belongs to $D_{0}\left[\mathcal{E}_{\Gamma}\right]$, using Lemma 7.7 with $g=g_{\Gamma}^{0}(a, *)$ and noting

$$
\int_{V} g_{\Gamma}^{0}(a, x) \mathcal{L}^{c}\left(H_{f^{2}}-\left(H_{f}\right)^{2}\right)(x) d \mu_{V}^{c}(x) \leq 2 g_{\Gamma}^{0}(a, a) \mathcal{E}_{\Gamma}\left(H_{f}\right)<+\infty,
$$

we obtain

$$
\begin{aligned}
H_{f^{2}}(a)-H_{f}(a)^{2} & =\mathcal{E}_{\Gamma}\left(H_{f^{2}}-\left(H_{f}\right)^{2}, g_{\Gamma}^{0}(a, *)\right) \\
& =\int_{V} g_{\Gamma}^{0}(a, x) \mathcal{L}^{c}\left(H_{f^{2}}-\left(H_{f}\right)^{2}\right)(x) d \mu_{V}^{c}(x) .
\end{aligned}
$$

In this way, we get

$$
H_{f^{2}}(a) \leq 2 g_{\Gamma}^{0}(a, a) \mathcal{E}_{\Gamma}\left(H_{f}\right)+H_{f}(a)^{2}, \quad a \in V .
$$

Finally we have

$$
\begin{aligned}
H_{f}(a)^{2} & \leq 2 u(a)^{2}+2\left(u(a)-H_{f}(a)\right)^{2} \\
& \leq 2 u(a)^{2}+2 \mathcal{E}_{\Gamma}\left(u-H_{f}, g_{\Gamma}^{0}(a, *)\right)^{2} \\
& \leq 2 u(a)^{2}+2 \mathcal{E}_{\Gamma}\left(u-H_{f}\right) \mathcal{E}_{\Gamma}\left(g_{\Gamma}^{0}(a, *)\right) \\
& =2 u(a)^{2}+2 g_{\Gamma}^{0}(a, a)\left(\mathcal{E}(u)-\mathcal{E}\left(H_{f}\right)\right) .
\end{aligned}
$$

Thus we obtain the required estimate. This completes the proof of Lemma 7.8 . 
Lemma 7.8 shows that the linear operator $T_{\mathcal{E}}$ from $D\left[\mathcal{L}_{\mathcal{E} ; \mu}\right]$ into $C\left(\partial \bar{V}^{N}(\mathcal{E})\right)$ extends uniquely to a bounded operator $\bar{T}_{\mathcal{E}}$ from $D[\mathcal{E}]$ into $L^{2}\left(\partial \bar{V}^{N}(\mathcal{E}), \omega\right)$ which sends bounded functions in $D[\mathcal{E}]$ into $L^{\infty}\left(\partial \bar{V}^{N}(\mathcal{E}), \omega\right)$, and any $u \in$ $D[\mathcal{E}]=H_{\mathcal{E}}+D_{0}\left[\mathcal{E}_{\Gamma}\right]$ can be written as

$$
u(x)=\int_{\partial \bar{V}^{N}(\mathcal{E})} \bar{T}_{\mathcal{E}} u d \omega_{x}+g(x), \quad g \in D_{0}\left[\mathcal{E}_{\Gamma}\right], \quad x \in V .
$$

Definition 7.1 We say that a function $u \in D[\mathcal{E}]$ has a normal derivative $\phi$ in $L^{2}\left(\partial \bar{V}^{N}(\mathcal{E}), \omega\right)$ if $\int\left|\mathcal{L}^{c} u\right| d \mu_{V}^{c}$ is finite and $u$ satisfies

$$
\mathcal{E}(u, v)=\int_{V} v \mathcal{L}^{c} u d \mu_{V}^{c}+\int_{\partial \bar{V}^{N}(\mathcal{E})}\left(\bar{T}_{\mathcal{E}} v\right) \phi d \omega
$$

for all bounded functions $v$ in $D[\mathcal{E}]$.

A normal derivative $\phi$ of $u$ if it exists is unique in $L^{2}\left(\partial \bar{V}^{N}, \omega\right)$. Moreover it depends on the choice of a reference point $o$, but the measure $\phi d \omega$ is independent.

In terms of Definition 7.1, it holds that a function $u \in D[\mathcal{E}]$ belongs to $D\left[\mathcal{L}_{\mathcal{E} ; \mu}\right]$ if and only if $\int_{V}\left|\mathcal{L}^{c} u(x)\right|^{2} \mu(x)^{-1} d \mu_{V}^{c}(x)<+\infty$ and $u$ has a normal derivative zero in $L^{2}\left(\partial \bar{V}^{N}(\mathcal{E}), \omega\right)$; in particular, for a function $f$ on a finite subset $K$ of $V$, the minimizer $H_{K ; f} \in D[\mathcal{E}]$ has a normal derivative zero in $L^{2}\left(\partial \bar{V}^{N}(\mathcal{E}), \omega\right)$.

Now we prove the following

Theorem 7.9 A function $\phi \in L^{2}\left(\partial \bar{V}^{N}(\mathcal{E}), \omega\right)$ is the normal derivative of a harmonic function in $D[\mathcal{E}]$ if and only if $\int_{\partial \bar{V}^{N}(\mathcal{E})} \phi d \omega=0$.

Proof. We are given $\phi \in L^{2}\left(\partial \bar{V}^{N}(\mathcal{E}), \omega\right)$. If it is the normal derivative of $h \in H_{\mathcal{E}}$, then $\int_{\partial \bar{V}^{N}(\mathcal{E})} \phi d \omega=\int_{\partial \bar{V}^{N}(\mathcal{E})} \phi \bar{T} 1 d \omega=\mathcal{E}(h, 1)=0$.

Suppose now that $\int_{\partial \bar{V}^{N}{ }_{(\mathcal{E})}} \phi d \omega=0$. Let $H_{o}=\left\{h \in H_{\mathcal{E}} \mid h(o)=0\right\}$. we define a functional on $H_{o}$ by assigning $\int_{\partial \bar{V}^{N}{ }_{(\mathcal{E})}} \phi \bar{T}_{\mathcal{E}} h d \omega$ to $h \in H_{o}$. Then this functional is bounded, since

$$
\begin{aligned}
\left|\int_{\partial \bar{V}^{N}(\mathcal{E})} \phi \bar{T}_{\mathcal{E}} h d \omega\right|^{2} & \leq \int_{\partial \bar{V}^{N}(\mathcal{E})} \phi^{2} d \omega \int_{\partial \bar{V}^{N}(\mathcal{E})}\left(\bar{T}_{\mathcal{E}} h\right)^{2} d \omega \\
& \leq 2 g_{\Gamma}^{0}(o, o) \int_{\partial \bar{V}^{N}(\mathcal{E})} \phi^{2} d \omega \mathcal{E}(h),
\end{aligned}
$$

where we used Lemma 7.8 . 
Therefore there exists a unique $h_{\phi} \in H_{o}$ such that $\mathcal{E}\left(h_{\phi}, h\right)=\int \phi \bar{T}_{\mathcal{E}} h d \omega$ for any $h \in H_{o}$. Any bounded $v \in D[\mathcal{E}]$ is decomposed as $v=h+g$, where $h \in H_{\mathcal{E}}$ and $g \in D_{0}\left[\mathcal{E}_{\Gamma}\right]$. Then we have

$$
\begin{aligned}
\mathcal{E}\left(h_{\phi}, v\right) & =\mathcal{E}\left(h_{\phi}, h\right)=\mathcal{E}\left(h_{\phi}, h-h(o)\right)=\int_{\partial \bar{V}^{N}(\mathcal{E})} \phi \bar{T}_{\mathcal{E}}(h-h(o)) d \omega \\
& =\int_{\partial \bar{V}^{N}{ }_{(\mathcal{E})}} \phi \bar{T}_{\mathcal{E}} h d \omega=\int_{\partial \bar{V}^{N}{ }_{(\mathcal{E})}} \phi \bar{T}_{\mathcal{E}} v d \omega .
\end{aligned}
$$

This shows that $h_{\phi}$ has a normal derivative $\phi$ in $L^{2}\left(\partial \bar{V}_{\mathcal{E}}^{N}, \omega\right)$. This completes the proof of Theorem 7.9.

By Theorem 7.9, we can deduce the following

Theorem 7.10 The space of functions $h$ in $H_{\mathcal{E}}$ with normal derivatives in $L^{2}\left(\partial \bar{V}_{\mathcal{E}}^{N}, \omega\right)$ is dense in $H_{\mathcal{E}}$.

Proof. Let $N H$ stand for the space above. Suppose $N H$ is not dense in $H_{\mathcal{E}}$. Then there exists $h \in H_{\mathcal{E}}$ such that $h \neq 0, h(o)=0$, and $\mathcal{E}\left(h, h^{\prime}\right)=0$ for all $h^{\prime} \in N H$. Since $\int \bar{T}_{\mathcal{E}} h d \omega=h(o)=0$, applying Theorem 7.9 to $\bar{T}_{\mathcal{E}} h$, we have $h^{\prime} \in N H$ such that $\bar{T}_{\mathcal{E}} h$ is the normal derivative of $h^{\prime}$. Therefore we have $0=\mathcal{E}\left(h, h^{\prime}\right)=\int\left(\bar{T}_{\mathcal{E}} h\right)^{2} d \omega$, and hence $h=0$. This is a contradiction and thus the proof is completed.

Now we shall prove the following

Theorem 7.11 Let $\mathcal{E}$ be a resistance form on a connected, infinite network $\Gamma=(V, E, r)$ that is nonparabolic.

(I) $\sup _{x, y \in V} R_{\mathcal{E}}(x, y)$ is finite if and only if every function in $D[\mathcal{E}]$ is bounded. Moreover if these are the cases, then $\partial \mathcal{R}(V, \mathcal{E})=\Delta(\mathcal{E})=\partial \bar{V}^{N}(\mathcal{E})$.

(II) The following conditions are mutually equivalent:

(i) $\sup _{x, y \in V} h_{x}^{\mathcal{E}}(y, y)$ is finite.

(ii) Every function in $H_{\mathcal{E}}$ is bounded.

(iii) For any $u \in D[\mathcal{E}], \bar{T}_{\mathcal{E}} u$ is continuous on $\Delta^{N}(\mathcal{E})$.

(iv) A nonnegative function $u$ in $D[\mathcal{E}]$ satisfying $\mathcal{L}^{c} u(x) \leq 0$ is bounded.

Moreover under these conditions, the projection $\rho$ restricted to the harmonic boundary $\Delta(\mathcal{E})$ onto $\Delta^{N}(\mathcal{E})$ induces a homeomorphism between them.

Proof. The assertion (I) and also the equivalence between the conditions (i) and (ii) in (II) can be deduced from the same reasons as in Theorem 4.2. Condition (iv) follows (ii) in (II), since for any $h \in H_{\mathcal{E}}$, we have $\mathcal{L}^{c} h^{2}(x)=$ 
$-2 \sum_{x \sim y}\left(h(x)-h(y)^{2} / r(x, y) \leq 0\right.$. In view of the maximum principle, we see that (iii) implies (ii). To prove the converse, we consider a subspace $\mathcal{A}_{\mathcal{E}}$ of $D[\mathcal{E}]$ which consists of functions extending continuously up to $\partial \bar{V}_{\mathcal{E}}^{N}$. Then $\mathcal{A}_{\mathcal{E}}$ is dense in $D[\mathcal{E}]$, because $\mathcal{A}_{\mathcal{E}}$ contains the domain $D\left[\mathcal{L}_{\mathcal{E} ; \mu}\right]$ of the Laplace operator $\mathcal{L}_{\mathcal{E} ; \mu}$ on $L^{2}(V, \mu)$, where $\mu$ is a finite measure on $V$ satisfying (3.7): $\iint R_{\mathcal{E}}^{2}(x, y) d \mu(x) d \mu(y)<+\infty$. Suppose that (ii), or (i) holds. Then there exists a constant $C$ such that for any $h \in H_{\mathcal{E}}$,

$$
\sup _{x \in V}|h| \leq C\left(\mathcal{E}(h)^{1 / 2}+\mid h(o \mid),\right.
$$

where $o$ is a fixed point of $V$. Thus we have

$$
\sup _{\xi \in \Delta_{N}(\mathcal{E})}\left|T_{\mathcal{E}} u(\xi)\right| \leq C^{\prime}\left(\mathcal{E}(u)^{1 / 2}+\mid u(0 \mid)\right.
$$

for some $C^{\prime}$ and all $u \in \mathcal{A}_{\mathcal{E}}$. This proves (iii), because $\mathcal{A}_{\mathcal{E}}$ is dense in $D[\mathcal{E}]$.

It remains to prove that (ii) implies (iv). Let $u$ be a nonnegative function in $D[\mathcal{E}]$ such that $\mathcal{L}^{c} u$ is nonpositive in $V$. Let $\left\{V_{n}\right\}$ be an exhaustion of $V$. We decompose $h$ on $V_{n}$ as follows: $u=h_{n}+g_{n}$, where $h_{n}$ is harmonic on $V_{n}$ and $g_{n}$ coincides with $u$ on the boundary of $V_{n}$. Then it follows from the maximum principle that $g_{n}$ is nonpositive. Letting $n$ go to infinity, $h_{n}$ and $g_{n}$ respectively converges to a function $h \in H_{\mathcal{E}}$ and $g \in D_{0}\left[\mathcal{E}_{\Gamma}\right]$ and we can express $u$ as $u=h+g$ on $V$. Since $g$ is nonpositive, $0 \leq u \leq h$, and by the assumption, $h$ is bounded, so that $u$ is bounded. This completes the proof of Theorem 7.11.

Remark 7.1 (i) Let $(\mathcal{F}, D[\mathcal{F}])$ be a Dirichlet form on a closed linear subspace of $L^{2}\left(\partial \bar{V}^{N}(\mathcal{E}), \omega\right)$ with $\mathcal{F}(1)=0$ and define a form $\left(\mathcal{E}_{\mathcal{F}}, D\left[\mathcal{E}_{\mathcal{F}}\right]\right)$ on $V$ by

$$
\mathcal{E}_{\mathcal{F}}(u)=\mathcal{E}(u)+\mathcal{F}\left(\bar{T}_{\mathcal{E}} u\right) ; D\left[\mathcal{E}_{\mathcal{F}}\right]=\left\{u \in D[\mathcal{E}] \mid \bar{T}_{\mathcal{E}} u \in D[\mathcal{F}]\right\} .
$$

Then $\mathcal{E}_{\mathcal{F}}$ is a resistance form on $\Gamma$. Moreover for a positive number $t$, we set $\mathcal{E}_{\mathcal{F} ; t}(u)=\mathcal{E}(u)+t \mathcal{F}\left(\bar{T}_{\mathcal{E}} u\right)$. Then the limit of the forms as $t \rightarrow+\infty$ also gives a resistance form on $\Gamma$.

(ii) If we restrict ourselves to a class of connected, infinite graphs $G=$ $(V, E)$ with bounded degree, the conditions in Proposition 4.1 and Theorem 7.11 for the resistance forms $\mathcal{E}_{G}$ of the graphs are invariant under quasi-isometries, after the result in [24] mentioned in the introduction.

7.4 Before exhibiting examples of the Kuramochi boundaries of infinite networks, we make some observations.

Let $\Gamma=(V, E, r)$ be a connected, infinite network and $\mathcal{E}$ a resistance form on $\Gamma$. Given an infinite subset $K$ of $V$, let $W=V \backslash K$ and $\Gamma_{W}=$ 
$\left(W, E_{W}, r_{W}\right)$ be the subnetwork of $\Gamma$ generated by $W$. We decompose $\Gamma_{W}$ into the connected components $\left\{\Gamma_{i}=\left(W_{i}, E_{i}, r_{i}\right)\right\}_{i \in I}$. For any $\Gamma_{i}$, we denote by $K_{i}$ (resp. $E_{i}^{\prime}$ ) the set of vertices of $K$ which are adjacent to some of $W_{i}$ (resp. the set of edges which connect $K_{i}$ to $W_{i}$ ). Then we get connected subnetworks $\tilde{\Gamma}_{i}=\left(\tilde{W}_{i}, \tilde{E}_{i}, \tilde{r}_{i}\right)$, where $\tilde{W}_{i}=W_{i} \cup K_{i}, \tilde{E}_{i}=E_{i} \cup E_{i}^{\prime}$ and $\tilde{r}_{i}$ is the restriction of $r$ to $\tilde{E}_{i}$.

Proposition 7.12 Let $\Gamma$ and $\tilde{\Gamma}_{i}=\left(\tilde{W}_{i}, \tilde{E}_{i}, \tilde{r}_{i}\right)(i \in I)$ be as above. Suppose that every $K_{i}$ is a finite subset. Let $\mathcal{E}$ be a resistance form on $\Gamma$ and $\Gamma_{K}^{*}=\left(K, E^{*}, r^{*}\right)$ the connected infinite network associated to the trace $\mathcal{E}_{K}^{*}$ of $\mathcal{E}$ on $K$. Then the inclusion map $\iota$ of $K$ to $V$ extends to a continuous map $\bar{\iota}$ of the Kuramochi compactification $\bar{K}^{N}\left(\mathcal{E}_{K}^{*}\right)$ of $\mathcal{E}_{K}^{*}$ into the Kuramochi compactification $\bar{V}^{N}(\mathcal{E})$ of $\mathcal{E}$ such that $\bar{\iota}$ sends injectively the boundary $\partial \bar{K}^{N}\left(\mathcal{E}_{K}^{*}\right)$ into the boundary $\partial \bar{V}^{N}(\mathcal{E})$. Moreover suppose that any $\tilde{\Gamma}_{i}$ is a finite subnetwork and the effective resistance $R_{\tilde{\Gamma}_{i}}$ of $\tilde{\Gamma}_{i}$ is uniformly bounded, that is, for some positive constant $C, R_{\tilde{\Gamma}_{i}}(x, y) \leq C$ for all $x, y \in \tilde{W}_{i}$ and $i$. Then $\bar{\iota}: \bar{K}^{N}\left(\mathcal{E}_{K}^{*}\right) \rightarrow \bar{V}^{N}(\mathcal{E})$ induces a homeomorphism between $\partial \bar{K}^{N}\left(\mathcal{E}_{K}^{*}\right)$ and $\partial \bar{V}^{N}(\mathcal{E})$ which maps $\Delta^{N}\left(\mathcal{E}_{K}^{*}\right)$ onto $\Delta^{N}(\mathcal{E})$.

Proof. Let $g_{z}(x, y)$ and $g_{c}^{*}(a, b)$ be respectively the Green functions of $\Gamma$ and $\Gamma_{K}^{*}$. Let $\phi_{z, x}(y)=g_{z}(x, y)$ for $x, z \in V$ and $\phi_{c, a}^{*}(b)=g_{c}^{*}(a, b)$ for $a, c \in K$. Then in view of Lemma 1.9 and (1.2), we see that

$$
\phi_{c, a}(b)=\phi_{c, a}^{*}(b), \quad b \in K .
$$

Moreover we claim that for $x \in W_{i}$ and $z \in W_{j}$,

$$
\phi_{z, x \mid K} \in Q\left(K, \mathcal{E}_{K}^{*}\right) .
$$

In fact, letting $H=H_{K ; \phi_{z, x \mid K}}$, we see that $H$ has a normal derivative zero in $L^{2}\left(\partial \bar{V}_{\mathcal{E}}^{N}, \omega\right)$ and satisfies

$$
\mathcal{E}_{K}^{*}\left(\phi_{z, x \mid K}, v\right)\left(=\mathcal{E}\left(H, H_{K ; v}\right)\right)=0
$$

for all $v \in D\left[\mathcal{E}_{K}^{*}\right]$ which vanish on $K_{i} \cup K_{j}$, because

$$
\mathcal{E}\left(H, H_{K ; v}\right)=\int H_{K ; v} \mathcal{L}^{c} H d \mu_{V}^{c}=\sum_{x \in K_{i} \cup K_{j}} v(x) \mathcal{L}^{c} H(x)=0 .
$$

In view of (7.1) and (7.2), we see that $\iota: K \rightarrow V$ extends to a unique continuous map of $\bar{K}\left(\mathcal{E}_{K}^{*}\right)$ to $\bar{V}^{N}(\mathcal{E})$ which sends injectively $\partial \bar{K}^{N}\left(\mathcal{E}_{K}^{*}\right)$ to $\partial \bar{V}^{N}(\mathcal{E})$. 
Suppose that $\bar{\iota}(\xi) \in \Delta^{N}(\mathcal{E})$. Let $u \in D_{0}\left[\mathcal{E}_{K}^{*}\right]$ and take a sequence of functions $u_{n}$ on $K$ with finite supports in such a way that $\mathcal{E}_{K}^{*}\left(u-u_{n}\right)=$ $\mathcal{E}\left(H_{K ; u}-H_{K ; u_{n}}\right)$ tends to 0 as $n \rightarrow \infty$. Since all $H_{K ; u_{n}}$ are finitely supported in $V$, we see that $H_{K ; u}$ belongs to $D_{0}\left[\mathcal{E}_{\Gamma}\right]$. Hence for any sequence $\left\{a_{n}\right\}$ in $K$ going to $\xi, u\left(a_{n}\right)=H_{K ; u}\left(\iota\left(a_{n}\right)\right)$ tends to 0 as $n \rightarrow \infty$, because of the assumption. This shows that $\xi \in \Delta^{N}\left(\mathcal{E}_{K}^{*}\right)$.

Now we assume that $R_{\tilde{\Gamma}_{i}}(x, y) \leq C$ for all $x, y \in \tilde{W}_{i}$ and $i$. Let $\eta$ be a point of $\partial \bar{V}^{N}(\mathcal{E})$ and $\left\{x_{n}\right\}$ a sequence in $V$ which converges to $\eta$. To show that $\bar{\iota}$ is surjective, we may assume that $x_{n}$ belongs to some $W_{i(n)}$ for all sufficiently large $n$. Take a point $a_{n}$ in $K_{i(n)}$. Then for all $z, x \in V$,

$$
\left|\phi_{z, x}\left(x_{n}\right)-\phi_{z, x}\left(a_{n}\right)\right|^{2} \leq C \mathcal{E}_{\tilde{\Gamma}_{i}}\left(\phi_{z, x \mid \tilde{W}_{i(n)}}\right),
$$

which tends to 0 as $n \rightarrow \infty$ if we fix $z$ and $x$. As a result we have $\phi_{z, x}(\eta)=$ $\lim _{n \rightarrow \infty} \phi_{z, x}\left(x_{n}\right)=\lim _{n \rightarrow \infty} \phi_{z, x}\left(a_{n}\right)$ for all $z, x \in V$. This implies that $a_{n}$ converges to $\eta$ as $n \rightarrow \infty$. Thus we have shown that $\bar{\iota}$ is surjective.

Finally let us prove that $\bar{\iota}(\xi) \in \Delta^{N}(\mathcal{E})$ if $\xi \in \Delta^{N}\left(\mathcal{E}_{K}^{*}\right)$. Let $\left\{x_{n}\right\}$ be a sequence in $V$ going to $\bar{\iota}(\xi)$ and $u$ a function in $D_{0}\left[\mathcal{E}_{\Gamma}\right]$. Take a sequence $\left\{u_{n}\right\}$ of functions with finite supports on $V$ in such a way that $\mathcal{E}\left(u-u_{n}\right)$ tends to 0 as $n \rightarrow \infty$. Since $\mathcal{E}_{K}^{*}\left(u_{\mid K}-u_{n \mid K}\right) \leq \mathcal{E}\left(u-u_{n}\right)$, we see that $u_{\mid K} \in D_{0}\left[\mathcal{E}_{K}^{*}\right]$. We may assume that $x_{n}$ belongs to some $W_{i(n)}$ for all sufficiently large $n$. By (7.3), we can take a sequence $\left\{a_{n}\right\}$ in $K$ in such a way that $a_{n} \in K_{i(n)}$ and $a_{n}$ tends to $\xi$ as $n \rightarrow \infty$. Then $u_{\mid K}\left(a_{n}\right)\left(=u\left(a_{n}\right)\right)$ goes to 0 and hence so is $u\left(x_{n}\right)$, since we have

$$
\left|u\left(x_{n}\right)-u\left(a_{n}\right)\right|^{2} \leq C \mathcal{E}_{\tilde{\Gamma}_{i(n)}}\left(u_{\mid \tilde{W}_{i(n)}}\right) .
$$

This completes the proof of Proposition 7.12.

Let $G=(V, E)$ be a connected, locally finite, infinite graph. For a positive integer $n$, we denote by $B_{n}=\left(V_{n}, E_{n}\right)$ the graph with the set of vertices $\left\{x, z_{1}, \ldots, z_{n}, y\right\}$ and the set of edges $\left\{\left|x z_{i}\right|,\left|z_{i} y\right|, i=1, \ldots, n\right\}$. By assigning a positive integer $\nu(e)$ to each edge $e \in E$ and replacing each edge $e=|x y| \in E$ with the graph $B_{\nu(e)}$, we obtain a connected, locally finite, infinite graph $G_{\nu}=\left(V_{\nu}, E_{\nu}\right)$, where $V$ is assumed to be a subset of $V_{\nu}$. We remark that two metric spaces $\left(V, d_{G}\right)$ and $\left(V_{\nu}, d_{G_{\nu}}\right)$ are quasi isometric in the sense of Gromov. A weight function $r_{\nu}$ on $E$ is defined by $r_{\nu}(e)=2 \nu(e)^{-1}$ for $e \in E$. Then we get a network $\Gamma=\left(V, E, r_{\nu}\right)$ so that the identity map of $V$ extends to a continuous map of $\partial \mathcal{R}(G)$ onto $\partial \mathcal{R}\left(V, \mathcal{E}_{\Gamma}\right)$. Moreover the inclusion map of $V$ into $V_{\nu}$ extends to a homeomorphism between $\partial \mathcal{R}\left(V, \mathcal{E}_{\Gamma}\right)$ and $\partial \mathcal{R}\left(G_{\nu}\right)$ which induces also a homeomorphism between the Kuramochi boundaries $\partial \bar{V}^{N}(G)$ and $\partial{\overline{V_{\nu}}}^{N}\left(G_{\nu}\right)$. For instance, we assume that a given 
graph $G=(V, E)$ is hyperbolic in the sense of Gromov and $0<e(G)<$ $2 \delta_{0}(G)$ (cf. Proposition 4.6). Then we choose a function $\nu: E \rightarrow \mathbb{Z}^{+}$such that $C^{-1} \exp (\eta n) \leq \nu(e) \leq C \exp (\eta n)$ if $e \in E(n)(n=1,2, \ldots)$, where $C$ is a constant greater than 1 and $\eta$ is a positive number less than $2 \delta_{0}(G)-e(G)$. Then it turns out that $\partial \mathcal{R}\left(G_{\nu}\right)=\partial{\overline{V_{\nu}}}^{N}\left(G_{\nu}\right)$ is homeomorphic to the Gromov boundary of $G$. Note that the degree of $G_{\nu}$ is unbounded.

Example 7.1 We are given a connected, locally finite, infinite graph $G=(V, E)$. First we take a family of locally finite, infinite graphs indexed by the set of the vertices $V$ of $G,\left\{G_{\alpha}=\left(V_{\alpha}, E_{\alpha}\right) \mid \alpha \in V\right\}$, in such a way that the effective resistance of $G_{\alpha}$ is uniformly bounded by a positive constant $C$, and each $G_{\alpha}$ admits no nonconstant harmonic functions of bounded Dirichlet sum. This implies that the Royden compactification of $G_{\alpha}$ for any $\alpha \in V$ consists of $V_{\alpha}$ and a single point $p_{\alpha}$. Secondly we choose a sequence $\left\{p_{\alpha ; n}\right\}$ of vertices in $V_{\alpha}$ tending to $p_{\alpha}$ for each $\alpha \in V$, and then for any edge $(\alpha, \beta) \in E$, we connect $V_{\alpha}$ with $V_{\beta}$ by a family of paths $\left(C_{\alpha \beta}, E_{\alpha \beta}\right)=\left\{\left(C_{\alpha \beta}^{(n)}, E_{\alpha \beta}^{(n)}\right) \mid n=\right.$ $1,2, \ldots\}$ joining $\left\{p_{\alpha ; n}\right\}$ to $\left\{p_{\beta ; n}\right\}$, that is,

$$
\begin{gathered}
C_{\alpha \beta}^{(n)}=\left\{p_{\alpha ; n}=x_{\alpha \beta ; 0}^{(n)}, x_{\alpha \beta ; 1}^{(n)}, \ldots, x_{\alpha \beta ; r(n)}^{(n)}=p_{\beta ; n}\right\} ; \\
E_{\alpha \beta}^{(n)}=\left\{\left(x_{\alpha \beta ; i}^{(n)}, x_{\alpha \beta ; i+1}^{(n)}\right) \mid i=0, \ldots, r(n)-1\right\},
\end{gathered}
$$

and the length $r(n)$ of the path $C_{\alpha \beta}^{(n)}$ is assumed to satisfy

$$
\sum_{n=1}^{\infty} \frac{1}{r(n)}=1
$$

Let $\tilde{V}=\left(\cup_{\alpha \in V} V_{\alpha}\right) \cup\left(\cup_{(\alpha, \beta) \in E} C_{\alpha \beta}\right)$ and $\tilde{E}=\left(\cup_{\alpha \in V} E_{\alpha}\right) \cup\left(\cup_{(\alpha, \beta) \in E} E_{\alpha \beta}\right)$. Then we get a connected, locally finite, infinite graph $\tilde{G}=(\tilde{V}, \tilde{E})$, the end compactification of which is the one-point compactification, that is, the metric graph $|\tilde{G}|$ is connected at infinity. Note that the degree of $\tilde{G}$ is bounded if those of $G$ and $G_{\alpha}$ for all $\alpha$ are bounded by a positive integer.

In what follows, we illustrate the Kuramochi boundary of $\tilde{G}$.

For a function $f$ on $\tilde{V}$, we say that $f$ is linear on $C_{\alpha \beta}^{(n)}$ if

$$
f\left(x_{\alpha \beta ; i}^{(n)}\right)=\frac{f\left(p_{\beta ; n}\right)-f\left(p_{\alpha ; n}\right)}{r(n)} i+f\left(p_{\alpha ; n}\right), \quad i=0,1, \ldots, r(n),
$$

and denote by $\Pi$ the space of functions on $\tilde{V}$ which are linear on all $C_{\alpha \beta}^{(n)}$. If a function $f$ belongs to $\Pi$, then we have

$$
\mathcal{E}_{\tilde{G}}(f)=\sum_{\alpha \in V} \mathcal{E}_{G_{\alpha}}\left(f_{\mid V_{\alpha}}\right)+\frac{1}{2} \sum_{\alpha, \beta \in V, \alpha \sim \beta} \sum_{n=1,2, \ldots} \frac{1}{r(n)}\left(f\left(p_{\alpha ; n}\right)-f\left(p_{\beta ; n}\right)\right)^{2} .
$$


To a function $u$ on $V$, we can assign a function $f_{u}$ of $\Pi$ such that $f_{u}=u(\alpha)$ on $V_{\alpha}$ for all $\alpha \in V$. Then it holds that

$$
\mathcal{E}_{G}(u)=\mathcal{E}_{\tilde{G}}\left(f_{u}\right)
$$

Moreover we see that $\mathcal{L}^{c} f_{u}(x)=\mathcal{L}^{c} u(\alpha) / r(n)$ if $x=p_{\alpha ; n}$, and $\mathcal{L}^{c} f_{u}(x)=0$ otherwise. It is possible to choose finite measures $\mu$ on $V$ and $\tilde{\mu}$ on $\tilde{V}$ satisfying (3.7) in such a way that $\tilde{\mu}\left(p_{\alpha ; n}\right)=\mu(\alpha) / r(n)$ for all $p_{\alpha ; n}$. Then it is evident that $f_{u} \in D\left[\mathcal{L}_{\tilde{\mu}}\right]$ if $u \in D\left[\mathcal{L}_{\mu}\right]$.

Now we are given $f \in D\left[\mathcal{E}_{\tilde{G}}\right]$. The restriction of $f$ to $V_{\alpha}, f_{\alpha}$, belongs to $D\left[\mathcal{E}_{G_{\alpha}}\right]$ and hence $f_{\alpha}(x)$ tends to a constant, denoted by $u_{f}(\alpha)$, as $x \in V_{\alpha} \rightarrow p_{\alpha}$. Note that

$$
\left|f_{\alpha}(x)-f_{\alpha}(y)\right|^{2} \leq C \mathcal{E}_{G_{\alpha}}\left(f_{\alpha}\right)
$$

for all $x, y \in V_{\alpha}$, so that we have

$$
\left|f_{\alpha}(x)-u_{f}(\alpha)\right|^{2} \leq C \mathcal{E}_{G_{\alpha}}\left(f_{\alpha}\right)
$$

for all $x \in V_{\alpha}$. Moreover in view of (7.4) and (7.6), we see that

$$
\mathcal{E}_{G}\left(u_{f}\right) \leq 3(C+1) \mathcal{E}_{\tilde{G}}(f) .
$$

Indeed, we have

$$
\begin{aligned}
\mathcal{E}_{G}\left(u_{f}\right)= & \frac{1}{2} \sum_{\alpha, \beta \in V, \alpha \sim \beta}\left(u_{f}(\alpha)-u_{f}(\beta)\right)^{2} \\
\leq & \frac{3}{2} \sum_{\alpha, \beta \in V, \alpha \sim \beta} \sum_{n=1,2, \ldots} \frac{1}{r(n)}\left(\left(u_{f}(\alpha)-f\left(p_{\alpha ; n}\right)\right)^{2}+\left(f\left(p_{\alpha ; n}\right)-f\left(p_{\beta ; n}\right)\right)^{2}\right. \\
& \left.+\left(f\left(p_{\beta ; n}\right)-u_{f}(\beta)\right)^{2}\right) \\
\leq & 3 C \sum_{\alpha \in V} \mathcal{E}_{G_{\alpha}}\left(f_{\alpha}\right)+\frac{3}{2} \sum_{\alpha, \beta \in V, \alpha \sim \beta} \sum_{n=1,2, \ldots} \frac{\left(f\left(p_{\alpha ; n}\right)-f\left(p_{\beta ; n}\right)\right)^{2}}{r(n)} \\
\leq & 3(C+1) \mathcal{E}_{\tilde{G}}(f) .
\end{aligned}
$$

Here we claim that $f \in D_{0}\left[\mathcal{E}_{\tilde{G}}\right]$ if and only if $u_{f}=0$. Indeed, for $f \in D_{0}\left[\mathcal{E}_{\tilde{G}}\right]$, we take a sequence of functions $f_{n}$ finitely supported on $\tilde{V}$ in such a way that $\lim _{n \rightarrow \infty} \mathcal{E}_{\tilde{G}}\left(f-f_{n}\right)=0$ and $\lim _{n \rightarrow \infty} f_{n}(x)=f(x)$ for each $x \in \tilde{V}$. Then in view of (7.6), we see that for all $\alpha \in V, u_{f}(\alpha)=\lim _{n \rightarrow \infty} u_{f-f_{n}}(\alpha)=0$. Now suppose that $u_{f}=0$. Let $f=h+g$, where $h \in H D\left[\mathcal{E}_{\tilde{G}}\right]$ and $g \in D_{0}\left[\mathcal{E}_{\tilde{G}}\right]$. Then $u_{h}=u_{f}=0$, so that $h(x)$ tends to zero as $x \in V \rightarrow \infty$. Thus the maximum principle implies that $h=0$. In this way, we have seen that the 
correspondence $f \rightarrow u_{f}$ gives rise to a linear isomorphism between $H D\left[\mathcal{E}_{\tilde{G}}\right]$ and $D\left[\mathcal{E}_{G}\right]$. In other words, for a function $u \in D\left[\mathcal{E}_{G}\right]$, let $f_{u}=H_{u}+g_{u}$, where $H_{u} \in H D\left[\mathcal{E}_{\tilde{G}}\right]$ and $g_{u} \in D_{0}\left[\mathcal{E}_{\tilde{G}}\right]$. Then assigning $H_{u}$ to $u$, we have a linear isomorphism between $D\left[\mathcal{E}_{G}\right]$ and $H D\left[\mathcal{E}_{\tilde{G}}\right]$. A quadratic form on $D\left[\mathcal{E}_{G}\right]$ is defined by

$$
\tilde{\mathcal{E}}(u, v)=\mathcal{E}\left(H_{u}, H_{v}\right), \quad u, v \in D\left[\mathcal{E}_{G}\right]
$$

Then we have

$$
\frac{1}{3(C+1)} \mathcal{E}_{G}(u) \leq \tilde{\mathcal{E}}(u) \leq \mathcal{E}_{G}(u), \quad u \in D\left[\mathcal{E}_{G}\right]
$$

The first inequality follows from (7.7) and the second one holds true, because $\mathcal{E}_{\tilde{G}}\left(H_{u}\right) \leq \mathcal{E}_{\tilde{G}}\left(f_{u}\right)=\mathcal{E}_{G}(u)$ by (7.5). From (7.8), we see that the effective resistance $R_{\tilde{\mathcal{E}}}$ of the resistance form $\tilde{\mathcal{E}}$ on $V$ is estimated as follows:

$$
R_{G}(\alpha, \beta) \leq R_{\tilde{\mathcal{E}}}(\alpha, \beta) \leq 3(C+1)\left(1+R_{G}(\alpha, \beta)\right), \quad \alpha, \beta \in V .
$$

In addition, we can observe that

$$
R_{G}(\alpha, \beta) \leq R_{\tilde{G}}(x, y) \leq 9(C+1)\left(1+R_{G}(\alpha, \beta)\right), \quad x \in V_{\alpha}, \beta \in V_{\beta} .
$$

The first inequality is a consequence from (7.5) and the second one can be verified as follows: for any $x \in V_{\alpha}$ and $y \in V_{\beta}$, and for any $f \in D\left[\mathcal{E}_{\tilde{G}}\right]$, letting $f^{\prime}$ be the function of $\Pi$ that is equal to $f$ on $\cup_{\alpha \in V} V_{\alpha}$, we get

$$
\begin{aligned}
|f(x)-f(y)|^{2} & =\left|f^{\prime}(x)-f^{\prime}(y)\right|^{2} \\
& =3\left(\left|f^{\prime}(x)-u_{f^{\prime}}(\alpha)\right|^{2}+\left|u_{f^{\prime}}(\alpha)-u_{f^{\prime}}(\beta)\right|^{2}+\left|u_{f^{\prime}}(\beta)-f^{\prime}(y)\right|^{2}\right) \\
& =3\left(C \mathcal{E}_{G_{\alpha}}\left(f_{\alpha}\right)+R_{G}(\alpha, \beta) \mathcal{E}_{G}\left(u_{f}\right)+C \mathcal{E}_{G_{\beta}}\left(f_{\beta}\right)\right) \\
& \leq 3\left(2 C+3 R_{G}(\alpha, \beta)(C+1)\right) \mathcal{E}_{\tilde{G}}\left(f^{\prime}\right) \\
& \leq 9(C+1)\left(1+R_{G}(\alpha, \beta)\right) \mathcal{E}_{\tilde{G}}(f) .
\end{aligned}
$$

Now for $u \in D\left[\mathcal{E}_{G}\right]$, we consider a function on $V$ defined by

$$
\Theta u(\alpha)=\sum_{n=1}^{\infty} \frac{H_{u}\left(p_{\alpha ; n}\right)}{r(n)}, \quad \alpha \in V .
$$

Then $\Theta u$ belongs to $D\left[\mathcal{E}_{G}\right]$. In fact, we have

$$
\begin{aligned}
(\Theta u(\alpha)-\Theta u(\beta))^{2} & =\left(\sum_{n=1}^{\infty} \frac{H_{u}\left(p_{\alpha ; n}\right)-H_{u}\left(p_{\beta ; n}\right)}{r(n)}\right)^{2} \\
& \leq \sum_{n=1}^{\infty} \frac{\left(H_{u}\left(p_{\alpha ; n}\right)-H_{u}\left(p_{\beta ; n}\right)\right)^{2}}{r(n)}=\mathcal{E}_{C_{\alpha \beta}}\left(H_{u \mid C_{\alpha \beta}}\right)
\end{aligned}
$$


and hence

$$
\mathcal{E}_{G}(\Theta u)=\frac{1}{2} \sum_{\alpha \sim \beta}(\Theta u(\alpha)-\Theta u(\beta))^{2} \leq \frac{1}{2} \sum_{\alpha \sim \beta} \mathcal{E}_{C_{\alpha \beta}}\left(H_{u \mid C_{\alpha \beta}}\right) \leq \mathcal{E}_{\tilde{G}}\left(H_{u}\right)=\tilde{\mathcal{E}}(u) .
$$

Obviously $\Theta 1=1$ and we can deduce that

$$
\tilde{\mathcal{E}}(u, v)=\mathcal{E}_{G}(u, \Theta v), \quad u, v \in D\left[\mathcal{E}_{G}\right],
$$

since we have

$$
\begin{aligned}
\tilde{\mathcal{E}}(u, v) & =\mathcal{E}_{\tilde{G}}\left(H_{u}, H_{v}\right)=\mathcal{E}_{\tilde{G}}\left(f_{u}, H_{v}\right) \\
& =\frac{1}{2} \sum_{\alpha \sim \beta}(u(\alpha)-u(\beta))(\Theta v(\alpha)-\Theta v(\beta))=\mathcal{E}_{G}(u, \Theta v) .
\end{aligned}
$$

Let

$$
\Theta_{\alpha}=\Theta \chi_{\alpha}, \quad \alpha \in V .
$$

Then we have $\tilde{\mathcal{E}}\left(\chi_{\alpha}, \chi_{\beta}\right)=-\frac{1}{2}\left(\mathcal{L}^{c} \Theta_{\alpha}(\beta)+\mathcal{L}^{c} \Theta_{\beta}(\alpha)\right)$. Letting

$$
\tilde{C}(\alpha, \beta)=\frac{1}{2}\left(\mathcal{L}^{c} \Theta_{\alpha}(\beta)+\mathcal{L}^{c} \Theta_{\beta}(\alpha)\right),
$$

we can express the form $\tilde{\mathcal{E}}$ as follows:

$$
\tilde{\mathcal{E}}(u, v)=\frac{1}{2} \sum_{\alpha, \beta \in V} \tilde{C}(\alpha, \beta)(u(\alpha)-u(\beta))(v(\alpha)-v(\beta)) .
$$

Let $\omega$ be the harmonic measure (relative to a fixed point $o$ of $\tilde{V}$ ) on the harmonic part of the Kuramochi boundary of $\tilde{G}, \Delta^{N}(\tilde{G})$, which includes the set of vertices $V$ of $G$ as an open dense subset. Then $\omega(V)=\sum_{\alpha \in V} H_{\chi_{\alpha}}(o)=1$, so that $\omega\left(\Delta^{N}(\tilde{G}) \backslash V\right)=0$. Finally we observe that for $u \in D[\tilde{\mathcal{E}}], H_{u}$ has a normal derivative $\phi d \omega$ in $L^{2}\left(\Delta^{N}(\tilde{G})\right)$ if and only if $u \in D\left[\mathcal{L}_{\omega}\right]$, and if these are the cases, then $\phi d \omega=\mathcal{L}_{\omega}(\Theta u) d \omega=\mathcal{L}^{c}(\Theta u) d \mu^{c}$ and it holds that

$$
\mathcal{E}_{\tilde{G}}\left(f, H_{u}\right)=\sum_{\alpha \in V} u_{f}(\alpha) \mathcal{L}^{c}(\Theta u)(\alpha), \quad f \in B D\left[\mathcal{E}_{\tilde{G}}\right] .
$$

Remark 7.2 In the case where the effective resistance of the form $\mathcal{E}_{G}$ is bounded from above by a positive constant, $\tilde{G}$ satisfies the conditions in Theorem 7.11 (II), the Kuramochi boundary of $\tilde{G}$ is homeomorphic to the Royden compactification of the metric graph $|G|$ associated to $G, \mathcal{R}(|G|)$, that is the union of $|G|$ and the Royden boundary of $G$, and furthermore the part of regular points $\Delta(\tilde{G})$ is homeomorphic to the Royden compactification of $G$. 


\section{References}

[1] Aronszajn, N.: Theory of reproducing kernels. Trans. Amer. Math. Soc. 68 (1950), 337-404.

[2] BekKa, M. E. B. And Valette, A.: Group cohomology, harmonic functions and the first $L^{2}$-Betti number. Potential Anal. 6 (1997), 313-326.

[3] Benjamini, I. and Schramm, O.: Harmonic functions on planar and almost planar graphs and manifolds, via circle packings. Invent. Math. 126 (1996), 565-587.

[4] Benjamini, I. And Schramm, O.: Random walks and harmonic functions on infinite planar graphs using square tilings. Ann. Probab. 24 (1996), $1219-1238$.

[5] Bérard, P., Besson, G. and Gallot, S.: On embedding Riemannian manifolds in a Hilbert space using their heat kernels. Prépublication de l'Institut Fourier, no. 109, 1988.

[6] Bérard, P., Besson, G. and Gallot, S.: Embedding Riemannian manifolds by their heat kernels. Geom. Funct. Anal. 4 (1994), 373-398.

[7] Bourdon, M. and Pajot, H.: Cohomologie $\ell_{p}$ et produits amalgamés. Geom. Dedicata 107 (2004), 85-98.

[8] Bourdon, M., Martin, F. And Valette, A.: Vanishing and nonvanishing for the first $L^{p}$-cohomology of groups. Comment. Math. Helv. 80 (2005), 377-389.

[9] Burago, D., Burago, Y. and Ivanov, S.: A Course in Metric Geometry. Graduate Studies in Mathematics 33. American Mathematical Society, Providence, RI, 2001.

[10] Cartier, P.: Fonctions harmonique sur un arbre. In Symposia Mathematica, vol. Ix (Convegno di Calcolo delle Probabilità, INDAM, Rome, 1971), 203-270. Academic Press, London, 1972.

[11] Cartwright, D. I. And Woess, W.: Infinite graphs with nonconstant Dirichlet finite harmonic functions. SIAM J. Discrete Math. 5 (1992), 380-385.

[12] Cartwright, D. I., Soardi, P. M. and Woess, W.: Martin and end compactifications for non-locally finite graphs. Trans. Amer. Math. Soc. 338 (1993), 679-693.

[13] Chandra, A. K., Raghavan, P., Ruzzo, W. L., Smolensky, R. and TIWARI, P.: The electrical resistance of a graph captures its commute and cover times. Comput. Complexity 6 (1996/1997), 312-340.

[14] Colin de Verdière, Y., Pan, Y. and Ycart, B.: Singular limits of Schrödinger operators and Markov processes. J. Operator Theory $\mathbf{4 1}$ (1999), 151-173.

[15] Coornaert, M., Delzant, T. and Papadopoulos, A.: Géométrie et théorie des groupes. Lecture Notes in Mathematics 1441. Springer-Verlag, Berlin, 1990. 
[16] Dal Maso, G.: An introduction to $\Gamma$-convergence. Progress in Nonlinear Differential Equations and Their Applications 8. Birkäuser, Boston, 1993.

[17] Davidoff, G., Sarnak, P. and Valette, A.: Elementary number theory, group theory, and Ramanujan graphs. London Mathematical Society Student Texts 55. Cambridge University Press, Cambridge, 2003.

[18] Doob, J. L.: Boundary properties for functions with finite Dirichlet integrals. Ann. Inst. Fourier (Grenoble) 12 (1962), 573-621.

[19] EleK, G.: The $\ell_{p}$-cohomology and the conformal dimension of hyperbolic cones. Geom. Dedicata 68 (1997), 263-279.

[20] Foster, R. M.: The average impedance of an electrical network. In Contributions to Applied Mechanics (Reissner Anniversary Volume), 333-340. J. W. Edwards, Ann Arbor, Michigan, 1948.

[21] Fukushima, M., Ōshima, Y. and Takeda, M.: Dirichlet forms and symmetric Markov processes. De Gruyter Studies in Mathematics 19. Walter de Gruyter, Berlin-New York, 1994.

[22] Grigorchuk, R. I. AND ŻUK, A.: On the asymptotic spectrum of random walks on infinite families of graphs. In Random walks and discrete potential theory (Cortona, 1997), 188-204. Sympos. Math. 39. Cambridge Univ. Press, Cambridge, 1999.

[23] Gromov, M.: Hyperbolic Groups. In Essays in group theory, 75-263. Math. Sci. Res. Inst. Publ. 8. Springer, New York, 1987.

[24] Hattori, T. And Kasue, A.: Dirichlet finite harmonic functions and points at infinity of graphs and manifolds. Proc. Japan Acad. Ser. A Math Sci. 83, 129-134.

[25] Hattori, T. And Kasue, A.: Functions of finite Dirichlet sums and compactifications of infinite graphs. In Probabilistic Approach to Geometry, 141-153. Advanced Studies in Pure Mathematics 57. Mathematical Society of Japan, Tokyo, 2010.

[26] Kasue, A.: Convergence of Riemannian manifolds and Laplace operators. I. Ann. Inst. Fourier (Grenoble) 52 (2002), 1219-1257.

[27] Kasue, A.: Convergence of Riemannian manifolds and Laplace operators. II. Potential Anal. 24 (2006), 137-194.

[28] Kasue, A.: Variational convergence of finite networks. Interdiscip. Inform. Sci. 12 (2006), 57-70.

[29] Kasue, A. And Kumura, H.: Spectral convergence of Riemannian manifolds. Tôhoku Math. J. 46 (1994), 147-179.

[30] Kasue, A. And Kumura, H.: Spectral convergence of Riemannian manifolds. II. Tôhoku Math. J. 48 (1996), 71-120.

[31] Kayano, T. and Yamasaki, M.: Some properties of Royden boundary of an infinite network. Mem. Fac. Sci. Shimane Univ. 22 (1988), 11-19.

[32] Kigami, J.: Analysis on fractals. Cambridge Tracts in Mathematics 143. Cambridge University Press, Cambridge, 2001. 
[33] Kuwae, K. And Shioya, T.: Convergence of spectral structures: a functional analytic theory and its applications to spectral geometry. Comm. Anal. Geom. 11 (2003), 599-673.

[34] Maeda, F.-Y.: Normal derivatives on an ideal boundary. J. Sci. Hiroshima Univ. Ser. A-I Math. 28 (1964), 113-131.

[35] Murakami, A.: Kuramochi boundaries of infinite networks and extremal problems. Hiroshima Math. J. 24 (1994), 243-256.

[36] Murakami, A. And Yamasaki, M.: An introduction of Kuramochi boundary of an infinite network. Mem. Fac. Sci. Eng. Shimane Univ. Ser. B Math. Sci. 30 (1997), 57-89.

[37] SoArdi, P. M.: Potential theory on infinite networks. Lecture Notes in Mathematics 1590. Springer-Verlarg, Berlin, 1994.

[38] Tetali, P.: Random walks and effective resistance of networks. J. Theoret. Probab. 4 (1991), no. 1, 101-109.

[39] Urakawa, H.: Heat kernel and Green kernel comparison theorems for infinite graphs. J. Funct. Anal. 146 (1997), 206-235.

[40] Yamasaki, M.: Discrete Dirichlet potentials on an infinite network. RIMS Kokyuroku 610 (1987), 51-66.

Recibido: 3 de septiembre de 2007

Revisado: 10 de abril de 2009

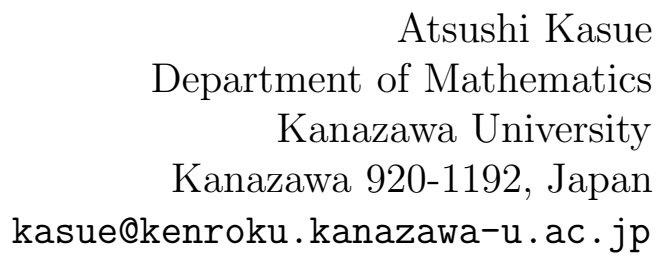

The author was partly supported by the Grant-in-Aid for Scientific Research (A) No. 19204004 of the Japan Society for the Promotion of Science. 